\title{
Regulation Mechanism of MYC Family Transcription Factors in Jasmonic Acid Signalling Pathway on Taxol Biosynthesis
}

\author{
Yunpeng Cui, Rongjia Mao, Jing Chen and Zhigang Guo * \\ Department of Chemical Engineering, Institute of Biochemical Engineering, Tsinghua University, Beijing 100084, \\ China; yunpengcui@126.com (Y.C.); gowersmao@126.com (R.M.); jingchen.titech@gmail.com (J.C.) \\ * Correspondence: guozhig@mail.tsinghua.edu.cn
}

Received: 19 February 2019; Accepted: 8 April 2019; Published: 14 April 2019

\begin{abstract}
Paclitaxel is an important anticancer drug. The phytohormone jasmonic acid can significantly induce the biosynthesis of paclitaxel in Taxus, but the molecular mechanism has not yet been resolved. To establish the jasmonic acid signalling pathway of Taxus media, based on the gene of the jasmonic acid signalling pathway of Arabidopsis thaliana, sequence analysis was performed to isolate the jasmonic acid signal from the transcriptome, a transcriptional cluster of pathway gene homologs and the full length of 22 genes were obtained by RACE PCR at $5^{\prime}$ and $3^{\prime}$ : two EI ubiquitin ligase genes, COI1-1 and COI1-2;7 MYC bHLH type transcription factor (MYC2, MYC3, MYC4, JAM1, JAM2, EGL3, TT8); 12 JAZ genes containing the ZIM domain; and MED25, one of the components of the transcriptional complex. The protein interaction between each were confirmed by yeast two hybridization and bimolecular fluorescence complementation based on similar genes interaction in Arabidopsis. A similar jasmonate signaling pathway was illustrated in T. media. All known paclitaxel biosynthesis genes promoters were isolated by genome walker PCR. To investigate the jasmonate signaling effect on these genes' expression, the transcription activity of MYC2, MYC3 and MYC4 on these promoters were examined. There are 12, 10 and 11 paclitaxel biosynthesis genes promoters that could be activated by MYC2, MYC3 and MYC4.
\end{abstract}

Keywords: jasmonate signalling; MYC transcription factors; taxol biosynthesis

\section{Introduction}

Paclitaxel is an important anticancer drug, which is mainly produced by chemical semi-synthesis by using paclitaxel, which is biosynthesized in Pacific yew. Due to its complex molecular structure, its raw materials have been dependent on Taxus biomass, and its biosynthesis and regulation have been studied [1]. The phytohormone jasmonic acid can significantly induce the biosynthesis of paclitaxel in Taxus, but the molecular mechanism has not yet been resolved [2-4].

The biosynthetic pathway of paclitaxel is very complex, involving at least 19 steps of enzyme-catalysed reactions, and the related enzymes and synthetic pathways have not been fully resolved. The synthetic pathway can be divided into the following four steps: taxane ring synthesis; side hydroxylation, acetylation and phenylacetylation modification; epoxidation modification at C5 position and oxidation at C9; and side chain at C13 position addition [5].

The precursor of taxadiene ring biosynthesis is derived from isopentenyl pyrophosphate (IPP) and dimethyl propylene pyrophosphate (DMAPP), the synthesis, mainly through the plastids of Taxus cells [5-7]. After ring formation, the biosynthesis process of paclitaxel includes various modifications to the sites on the ring, including hydroxylation, acetylation and benzoylation modification, epoxidation at the $\mathrm{C} 5$ position, and oxidative modification at the C9 position [7]. 
The known modification process is first hydroxylation of the taxane-4(5), 11(12)-diene at the $5 \alpha$ position. This is the rate-limiting step for paclitaxel synthesis [5]. Subsequently, there is hydroxylation of $1 \beta(1 \beta \mathrm{OH}), 2 \alpha(2 \alpha \mathrm{OH}), 7 \beta(7 \beta \mathrm{OH}), 9 \alpha(9 \alpha \mathrm{OH}), 10 \beta(10 \beta \mathrm{OH}), 13 \alpha(13 \alpha \mathrm{OH})$, acetylation of $5 \alpha(\mathrm{TBT})$ and $10 \beta$ (TAT), and phenylacetylation of $2 \alpha$ (DBAT). In addition to the $1 \beta$ and $9 \alpha$ position hydroxylases, the genes of other enzymes have been confirmed [5]. Oxidation at the $9 \alpha$ position and epoxidation between C4, C5 and C20 occur after the desired modification, resulting in the formation of baccatin III (see Figure 1), but the genes in both steps have not been isolated [5]. At least 11 enzymes are involved in the various modification processes, but the exact order has not been determined. Finally, baccatin III binds to the side chain under the catalysis of the enzyme, and undergoes hydroxylation of the opposite side chain and phenylacetylation to form the final product, paclitaxel. Phenylalanine oxidase (PAM), phenylpropionyl-CoA transferase (BAPT) at position C13, and phenylacetylase (DBTNBT) in the side chain have been identified in this process $[5,8]$.

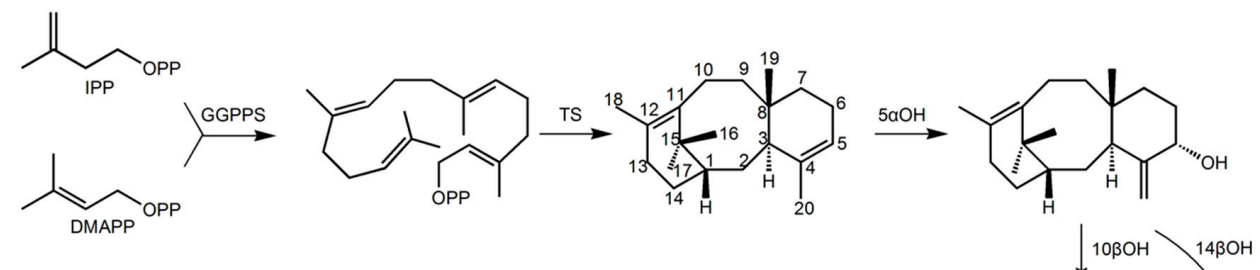

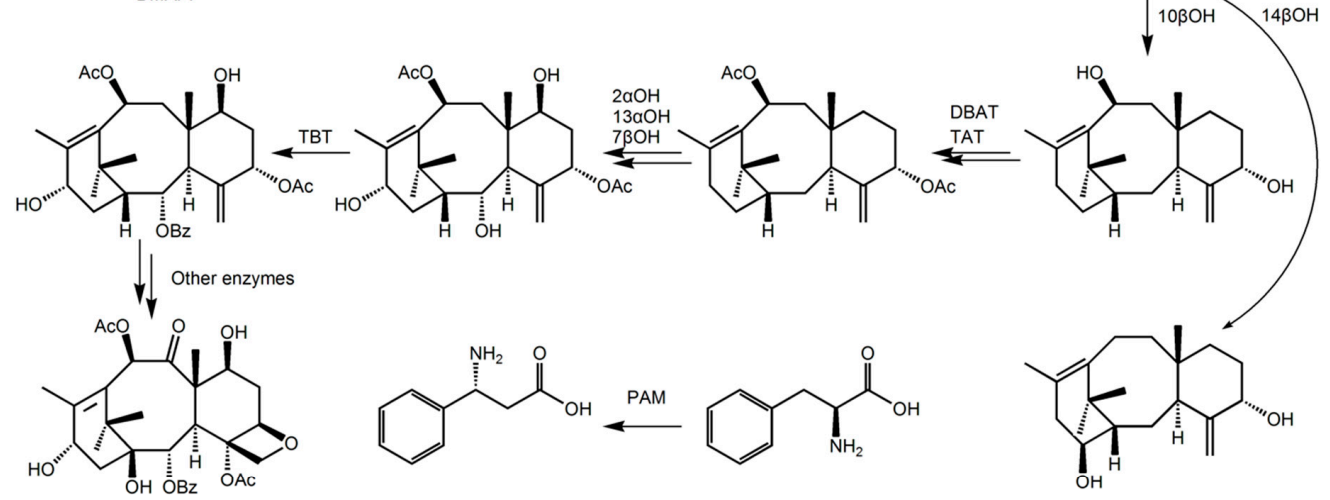

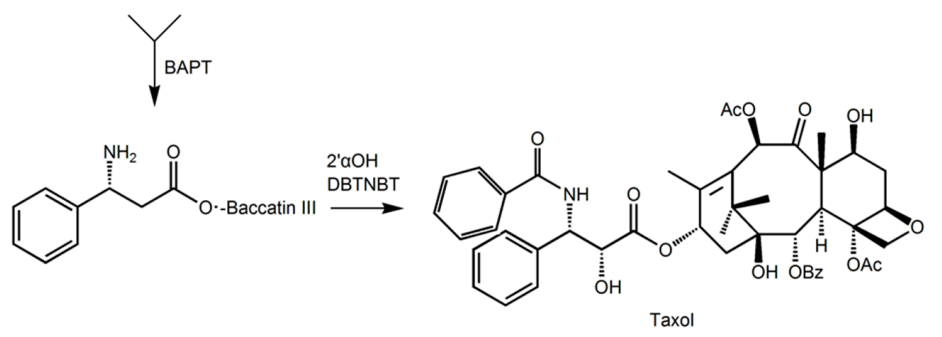

Figure 1. Schematic diagram of the main steps of the paclitaxel biosynthesis pathway. The short list is as follows: GGPPS, di-geranyl pyrophosphate synthase; TS, taxadiene synthase; $5 \alpha \mathrm{OH}$, taxadiene $5 \alpha$ hydroxylase; $10 \beta \mathrm{OH}$, Taxol $10 \beta$ hydroxylase; $14 \beta \mathrm{OH}$, violet Cedar $14 \beta$ hydroxylase; TAT, taxadiene-5 $\alpha$-ol-acetyltransferase; DBTNBT, $3^{\prime}-\mathrm{N}$-dephenylated paclitaxel-N-benzoyltransferase; TBT, taxane $2 \alpha$-O-phenylacetyltransferase; DBAT, 10-deacetylbaccatin III-10-O-acetyltransferase; $13 \alpha \mathrm{OH}$, taxane $13 \alpha$ hydroxylase; BAPT, phenylpropionyltransferase; $2 \alpha \mathrm{OH}$, Taxol $2 \alpha$ hydroxylase; $7 \beta \mathrm{OH}$, paclitaxel $7 \beta$ hydroxylase; PAM, phenylalanine isomerase; $2^{\prime} \alpha \mathrm{OH}$, paclitaxel $2^{\prime} \alpha$ hydroxylase.

The biosynthesis step of paclitaxel is very complicated, and it is very difficult to directly increase the expression of the biosynthetic pathway gene to increase the paclitaxel content. As the analysis of all synthetic pathways is not complete, the metabolic pathways cannot be precisely regulated to control the metabolic flux. In this case, for such a complex metabolic pathway, a better choice for regulating genes in the synthetic pathway is to regulate upstream transcription factors, thereby comprehensively regulating the expression of genes in the synthetic pathway [9]. It is important to analyse the transcription factors that regulate the expression of paclitaxel synthesis pathway genes. 
Transcription factors regulating Taxol biosynthesis in Taxus are being closely studied [2-4]. Previous studies have used two methods to isolate related transcription factors: one is to first isolate the promoter of the key gene of the Taxol synthesis pathway, and then to study the transcription factor that can be combined with the promoter; the other is to study jasmonic acid-induced transcription factors in order to analyse whether it can regulate gene expression of paclitaxel.

In the transcription factor-related studies in Taxus, no relevant research results of jasmonic acid signaling pathways in model plants have been used. There is also no systematic and in-depth discussion of the signaling pathway of jasmonic acid in Taxus. Li et al. used high-throughput sequencing to study the transcriptome of jasmonic acid treatment for $16 \mathrm{~h}$, and obtained an AtJAZ-like gene induced by jasmonic acid, and a similar AtMYC2 gene, but no in-depth study [10]. In the transcriptome sequencing study published by the Sun et al., the related gene expression after eight days of jasmonic acid treatment was involved, but no jasmine similar to that in the model plants induced in the early stage of jasmonic acid treatment was involved in the ketoacid signalling pathway transcription factor [11]. Lenka et al. published article on AtMYC2-like genes [8]; except for the TcMYC2 gene, which is known to have a full-length gene, other transcription factors were not found to be similar to AtMYC2 in model plants. The AtJAZ-binding region also does not contain active regions that activate transcription. Maybe due to the shortcomings of the adopted technical methods, the conclusions obtained were also different from those of similar genes in model organisms [12].

In this study, high-throughput sequencing was used to obtain transcription factors after an early induction with jasmonic acid. In our previous study, the sequence of several genes related to the paclitaxel synthesis pathway and jasmonic acid signaling pathway were obtained by sequencing analysis of the transcriptome and expression profile of Taxus. Based on this, the full-length sequence of jasmonic acid signalling pathway-related transcription factors in T. media was obtained by sequence comparison analysis and RACE PCR. It includes sequences similar to the important genes of the jasmonic acid signalling pathways such as AtCOI1, AtMYC2, AtJAZ and AtMED25 (At, Arabidopsis thaliana, if the source of the gene is not clearly prefixed, it is from the T. media). The interaction between JAZ and MYC transcription factors and other genes was studied, and the jasmonic acid signalling pathway in Taxus was confirmed. The above work further studied the regulation of the promoter of the paclitaxel synthesis pathway gene by the MYC transcription factor, and elucidated the regulation mechanism of the transcription factor of the jasmonic acid signaling pathway on the biosynthesis pathway of paclitaxel.

We also found that some of the genes involved in the paclitaxel synthesis pathway were highly expressed by jasmonic acid at $0.5 \mathrm{~h}$, similar to the transcription factors of the jasmonic acid signalling pathway and the expression of self-synthesis-related genes, but faster. Expression of anthocyanin synthesis-related genes regulated by the jasmonic acid signalling pathway was noted at three hours [13]. This indicated that the jasmonic acid signalling pathway plays an important role in regulating the gene expression of the paclitaxel synthesis pathway.

To analyse the mechanism of jasmonic acid signalling pathway specifically regulating the paclitaxel synthesis pathway gene, 14 promoters of paclitaxel synthesis pathway gene were isolated, and five MYC transcription factors were verified by fluorescence activity assay in Arabidopsis protoplasts. Regulation of the promoter of the paclitaxel biosynthetic pathway gene and the transcription factor mutants with application value were designed, which lays the foundation for the future construction of a high-yield paclitaxel-based Taxus cell line.

\section{Results}

\subsection{Identification and Analysis of the Full-Length Gene of Jasmonic Acid Signalling Path of Taxus}

\subsubsection{JAZ}

At least eight JAZ-containing ZIM domains were obtained in the differentially expressed genes by the transcriptome sequencing. For comprehensive detection of JAZ that may be contained in the transcriptome, BLAST analysis was performed using the full length of the Arabidopsis AtJAZ1 gene and 
the eight JAZs with reference to the Tm_ZK transcriptome, and the threshold of the E value was set to 0.01. Finally, 16 transcription cluster sequences which may be JAZ were obtained, as shown in Table 1 .

Table 1. List of suspected JAZ genes in the Tm_ZK transcriptome.

\begin{tabular}{lcccccc}
\hline \multirow{2}{*}{ Unigene ID } & \multirow{2}{*}{$\begin{array}{c}\text { Hit Gene } \\
\text { Description }\end{array}$} & \multirow{2}{*}{ Similarity (\%) } & \multicolumn{4}{c}{ RPKM } \\
\cline { 4 - 7 } & - & - & 11.1 & 18.8 & 99.8 & 135.7 \\
\hline comp47611_c0 & - & - & 0.2 & 0.2 & 2.9 & 1.5 \\
comp60304_c0 & - & - & 0.3 & 1.2 & 51.4 & 41.1 \\
comp64513_c0 & - & 41.94 & 0.9 & 9.1 & 131.5 & 81.3 \\
comp65899_c0 & Protein TIFY 9; & 40.66 & 0.1 & 1.2 & 25.5 & 2.9 \\
comp66635_c0 & Protein TIFY 9; & 32.52 & 40.4 & 92.2 & 419.4 & 220.0 \\
comp66717_c0 & Protein TIFY 9; & - & 0.4 & 0.9 & 27.3 & 1.0 \\
comp68543_c0 & - & - & 0.1 & 10.8 & 97.8 & 2.5 \\
comp70749_c0 & - & 28.37 & 45.1 & 42.1 & 49.0 & 63.0 \\
comp73423_c0 & Protein TIFY 6B; & 42.11 & 17.6 & 42.9 & 177.5 & 85.9 \\
comp73780_c0 & Protein TIFY 9; & 41.03 & 20.5 & 28.8 & 111.3 & 116.0 \\
comp73885_c0 & Protein TIFY 10B; & - & 0.3 & 14.0 & 101.3 & 2.7 \\
comp75328_c0 & - & 40.22 & 2.6 & 10.1 & 122.8 & 11.1 \\
comp76274_c0 & Protein TIFY 9; & 30.12 & 36.9 & 49.0 & 140.3 & 71.3 \\
comp76841_c0 & Protein TIFY 6B; & 35 & 0.2 & 3.1 & 34.2 & 2.3 \\
comp77597_c0 & Protein TIFY 6B; & 33.01 & 0.1 & 2.8 & 24.4 & 0.6 \\
comp78571_c0 & Protein TIFY 6B; & & & & & $\mathbf{~}$ \\
\hline
\end{tabular}

For the 16 possible JAZs, specific primers were designed and the full length of the gene was obtained by RACE PCR at the $3^{\prime}$ and $5^{\prime}$ ends. Three of the transcription clusters comp60304_c0, comp64513_c0, and comp68543_c0 failed to obtain a full-length sequence. The other 13 obtained the full length sequence, and wherein comp70749_c0 was a non-coding sequence. The remaining 12 transcription clusters obtained the encoded amino acid sequence-see Table 2.

Table 2. List of full-length JAZ genes isolated from Taxus.

\begin{tabular}{cccccc}
\hline \multirow{2}{*}{ Unigene ID } & \multirow{2}{*}{ Name } & Length & \multicolumn{3}{c}{ Folds } \\
\cline { 4 - 6 } & & & $\mathbf{0 . 5} \mathbf{h}$ & $\mathbf{3 ~ h}$ & $\mathbf{2 4} \mathbf{~ h}$ \\
\hline comp47611_c0 & JAZ11 & 171 & 1.7 & 9.0 & 12.2 \\
comp65899_c0 & JAZ7 & 142 & 10.1 & 146.1 & 90.3 \\
comp66635_c0 & JAZ12 & 200 & 12.0 & 255.0 & 29.0 \\
comp66717_c0 & JAZ6 & 205 & 2.3 & 10.4 & 5.4 \\
comp73423_c0 & JAZ4 & 393 & 0.9 & 1.1 & 1.4 \\
comp73780_c0 & JAZ5 & 182 & 2.4 & 10.1 & 4.9 \\
comp73885_c0 & JAZ10 & 168 & 1.4 & 5.4 & 5.7 \\
comp75328_c0 & JAZ1 & 225 & 46.7 & 337.7 & 9.0 \\
comp76274_c0 & JAZ9 & 202 & 3.9 & 47.2 & 4.3 \\
comp76841_c0 & JAZ3 & 448 & 1.3 & 3.8 & 1.9 \\
comp77597_c0 & JAZ8 & 530 & 15.5 & 171.0 & 11.5 \\
comp78571_c0 & JAZ2 & 201 & 28.0 & 244.0 & 6.0 \\
\hline
\end{tabular}

Homology analysis and domain analysis in the amino acid sequence were performed (see Figures 2a and A1), and the 12 genes were named JAZ1 to JAZ12.

The primers were designed to amplify the 12 full-length genes, and the cDNA was used as a template for PCR. The bands obtained from the cloning were sequenced to obtain 18 sequences. Among the 18, JAZ1, JAZ2, JAZ9, JAZ11 and JAZ12 all contained varying cleavages. There were three in JAZ2, and the other had two coding sequences (see the Appendices A and B for the specific sequences). Similar to the variable cleavage of the JAZ gene in model plants, it is important for the diversity of the regulatory functions of the JAZ gene. 

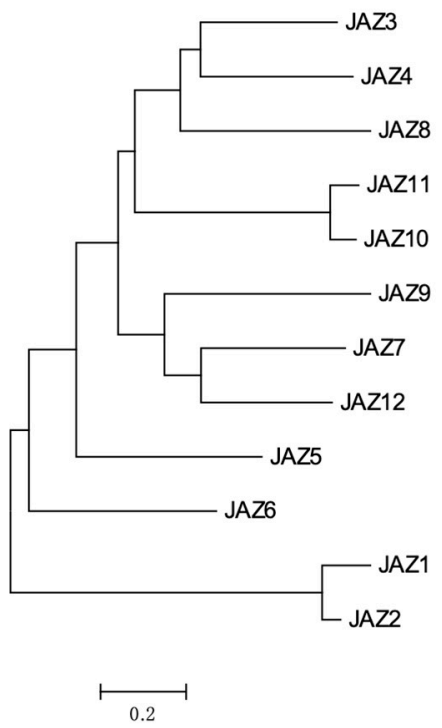

a

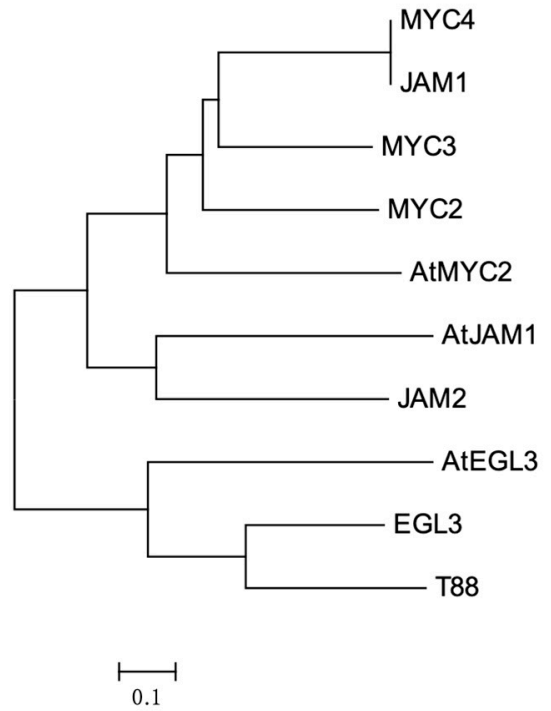

b

Figure 2. (a) analysis of JAZ gene evolutionary tree in Taxus; (b) results of phylogenetic tree analysis of MYC transcription factors in Taxus.

\subsubsection{COI1}

BLAST alignment analysis of the Tm_ZK transcriptome revealed two AtCOI1-like genes, named COI1. To detect the AtCOI1-like gene that may be contained in the transcriptome, BLAST analysis was performed using the full length of Arabidopsis AtCOI1 and the above two COI1 with reference to transcriptome, and the threshold of the E value was set to 0.00001 . Only the two sequences were obtained, and comp78658_c0 and comp69536_c0 may be the transcription cluster sequence of COI1.

For the two possible COI1, specific primers were designed and the full length was obtained by RACE PCR at the $3^{\prime}$ end and $5^{\prime}$ end. It was found that the two COI 1 were actually the full length of the single COI1 gene.

Specific primers to amplify COI1 were produced, and the two highly similar COI1, named COI1-1 and COI1-2, were cloned. Homology analysis was performed with AtCOI1 in Arabidopsis, and the results are shown in Figure A2. There were only 10 amino acid differences between COI1-1 and COI1-2, and most of them were not conservative amino acids. Only the amino acid at position 363 was a conservative amino acid variation.

\subsubsection{MYC Transcription Factor}

The MYC transcription factor belongs to the bHLH type. Ten bHLH-type transcription factors were obtained in the differentially expressed genes by transcriptome sequencing analysis. For comprehensive detection of MYC-like transcription factors that may be contained in the transcriptome, BLAST analysis was performed using the transcription factors of Arabidopsis AtMYC2 full length and the $10 \mathrm{bHLH}$ types, and the threshold of the E value was set to 0.01 . Finally, 19 possible transcription clusters were found, and homology analysis was performed to obtain full length of RACE for seven transcription clusters of comp78752_c0, comp74670_c0, comp77888_c0, comp77131_c0, comp75263_c0, comp77266_c0, and comp78399_c0. It was found that comp75263_c0 and comp78752_c0 were the sequences at both ends of the same gene (Table 3).

The primers were then designed and cloned using a high-fidelity enzyme to confirm the final gene sequence. The comp77888_c0 was amplified in both cDNA and genomic DNA. In addition to the predicted length bands, a shorter band was obtained. The truncated comp77888_c0 we named comp77888s_c0, so we finally isolated and identified 7 AtMYC2 similar transcription factors in T.media: 
comp74670_c0, comp78752_c0, comp77888_c0, comp77888s_c0, comp77131_c0, comp78399_c0 and comp77266_c0 (see the Appendices A and B for the sequence).

Table 3. List of MYC transcription factors in taxus.

\begin{tabular}{cccccc}
\hline \multirow{2}{*}{ Unigene ID } & \multirow{2}{*}{ Name } & \multirow{2}{*}{ AA Length } & \multicolumn{3}{c}{ Folds } \\
\cline { 4 - 6 } & & & $\mathbf{0 . 5} \mathbf{~ h}$ & $\mathbf{3 ~ h}$ & $\mathbf{2 4} \mathbf{~ h}$ \\
\hline comp74670_c0 & MYC2 & 662 & 1.35 & 1.72 & 1.05 \\
comp77131_c0 & JAM2 & 587 & 1.54 & 4.63 & 1.64 \\
comp78752_c0 & MYC3 & 843 & 1.58 & 3.33 & 1.63 \\
comp77888_c0 & MYC4 & 634 & 1.78 & 3.44 & 1.91 \\
comp78399_c0 & EGL3 & 686 & 0.92 & 0.96 & 1.24 \\
comp77266_c0 & TT8 & 734 & 0.96 & 1.71 & 1.04 \\
comp77888s_c0 & JAM1 & 566 & - & - & - \\
\hline
\end{tabular}

After homology analysis and domain identification (Figures $2 b$ and A3), the seven transcription factors were named MYC2, MYC3, MYC4, JAM1, JAM2, EGL3 and TT8.

Among them, MYC2, MYC3, MYC4 and JAM1 had higher similarity with AtMYC2 in Arabidopsis (Figure $2 b$ ), which may be the transcription factor of the core jasmonic acid signalling pathway. The gene expression levels of MYC2, MYC3, and MYC4 were also induced by jasmonic acid, especially MYC3 and MYC4, which were similar to those in the model plants. However, JAM1, which is highly homologous to MYC4, lacked a domain that binds to JAZ and may be a transcription factor that inhibits jasmonic acid signalling.

JAM2 is similar to Arabidopsis thaliana's inhibitory bHLH-type transcription factor AtJAM1, which is also upregulated by the induction of methyl jasmonate treatment, which may be another transcription factor that inhibits the jasmonic acid signalling pathway.

The similarity of transcription factors between TT8 and EGL3 and the jasmonic acid signalling pathway core in model plants was low, and the amino acid sequence of the relevant domain was also different, which is similar to the transcription factor sequence in the regulation of anthocyanin synthesis in model plants. Like the model plants and not induced by the jasmonic acid signal, it is likely to be a transcription factor involved in the regulation of anthocyanin metabolism in Taxus.

\subsubsection{MED25}

In model plants, a transcription factor such as AtMYC2 binds to AtMED25 to recruit RNA polymerase and initiate transcription of a downstream gene of the jasmonic acid signal [14]. At MED25 is an important gene for transcription initiation of jasmonic acid downstream pathway genes and plays an important role in the jasmonic acid signalling pathway. The unique and full length MED25 was obtained in the Taxus transcriptome by BLAST analysis. Primers were designed and amplified with high fidelity enzyme to obtain MED25. See the Appendices A and B for the sequence.

\subsection{Interaction between Jasmonic Acid Signalling Pathway Genes in Taxus}

The jasmonic acid signal is transmitted from the receptor to the protein through the interaction between the proteins. It is necessary to further investigate and detect the jasmonic acid signal by exploring whether the genes of the jasmonic acid signalling pathway are interacting, and to study JAZ and COI1, JAZ and MYC, JAZ and JAZ, MYC and MYC, MYC and MED25 protein interactions, determine whether it is the same as in the model plants, and then construct the jasmonic acid signal in the Taxus.

Since the interaction of the genes has been verified by two methods in model plants, in this study, to detect the interaction between related proteins, the yeast two-hybrid method was used to obtain preliminary interactions between proteins. 


\subsubsection{JAZ and COI1}

Jasmonyl isoleucine can mediate the binding of JAZ to COI1, thereby ubiquitin-labelling JAZ and degrading JAZ-released transcription factors. The combination of AtJAZ and AtCOI1 is an important basis for jasmonic acid signal reception in Arabidopsis. This study analysed $12 \mathrm{JAZ}$ and 2 COI1 from the Taxus, and the analog of coronatine, jasmonyl isoleucine. The results are shown in Table 4 ("-"represents no signal, "+" represents a signal, the number of "+"represents the signal's strength).

Table 4. Yeast two-hybrid analysis. The results of the interaction between JAZ and COI1.

\begin{tabular}{cccccccccccccc}
\hline \multirow{2}{*}{$\begin{array}{c}\text { Cor } \\
(\mu \mathbf{M})\end{array}$} & \multirow{2}{*}{ BD } & \multicolumn{10}{c}{ pB42AD, Prey(AD) } \\
\cline { 3 - 13 } & & JAZ1 & JAZ2 & JAZ3 & JAZ4 & JAZ5 & JAZ6 & JAZ7 & JAZ8 & JAZ9 & JAZ10 & JAZ11 & JAZ12 \\
\hline \multirow{2}{*}{0} & COI1-1 & - & - & - & - & - & - & - & ++ & - & - & - & - \\
& COI1-2 & - & - & - & - & - & - & - & - & ++ & - & - & - \\
\hline \multirow{2}{*}{10} & COI1-1 & - & - & - & - & - & - & - & ++ & - & - & - & - \\
& COI1-2 & - & - & - & - & - & - & - & - & ++ & - & - & + \\
\hline \multirow{2}{*}{30} & COI1-1 & - & - & - & - & - & + & - & ++ & + & - & - & + \\
& COI1-2 & - & - & - & - & - & - & + & + & ++ & + & - & ++ \\
\hline
\end{tabular}

In the absence of coronatine, COI1 interacts with JAZ8 and JAZ9. When using $10 \mu \mathrm{M}$ coronatine, COI1 can interact with JAZ8, JAZ9 and JAZ12 to increase the concentration of coronin to $30 \mu \mathrm{M}$. COI1 can be combined with JAZ6, JAZ7, JAZ8, JAZ9, JAZ10 and JAZ12 interaction.

There is also a similar combination of JAZ protein and COI1 protein in the Taxus. However, it is different from the results of the model plant Arabidopsis.

\subsubsection{JAZ and MYC}

In model plants, AtJAZ binds to AtMYCs, thereby inhibiting the transcriptional activity of AtMYCs. In this study, the binding of all JAZ to MYC in Taxus was analysed by the yeast two-hybrid method, and the interaction of some JAZ and MYC in tobacco was verified by using bimolecular fluorescence complementation (see Figure 3).

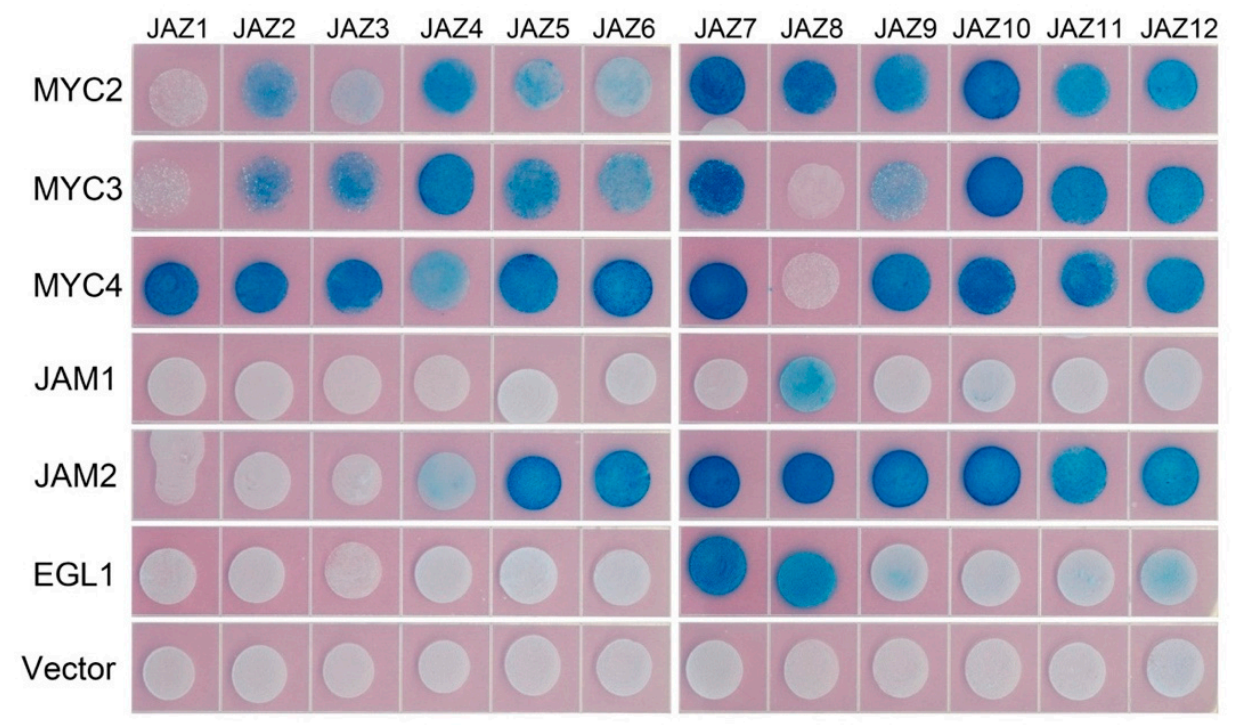

(a)

Figure 3. Cont. 


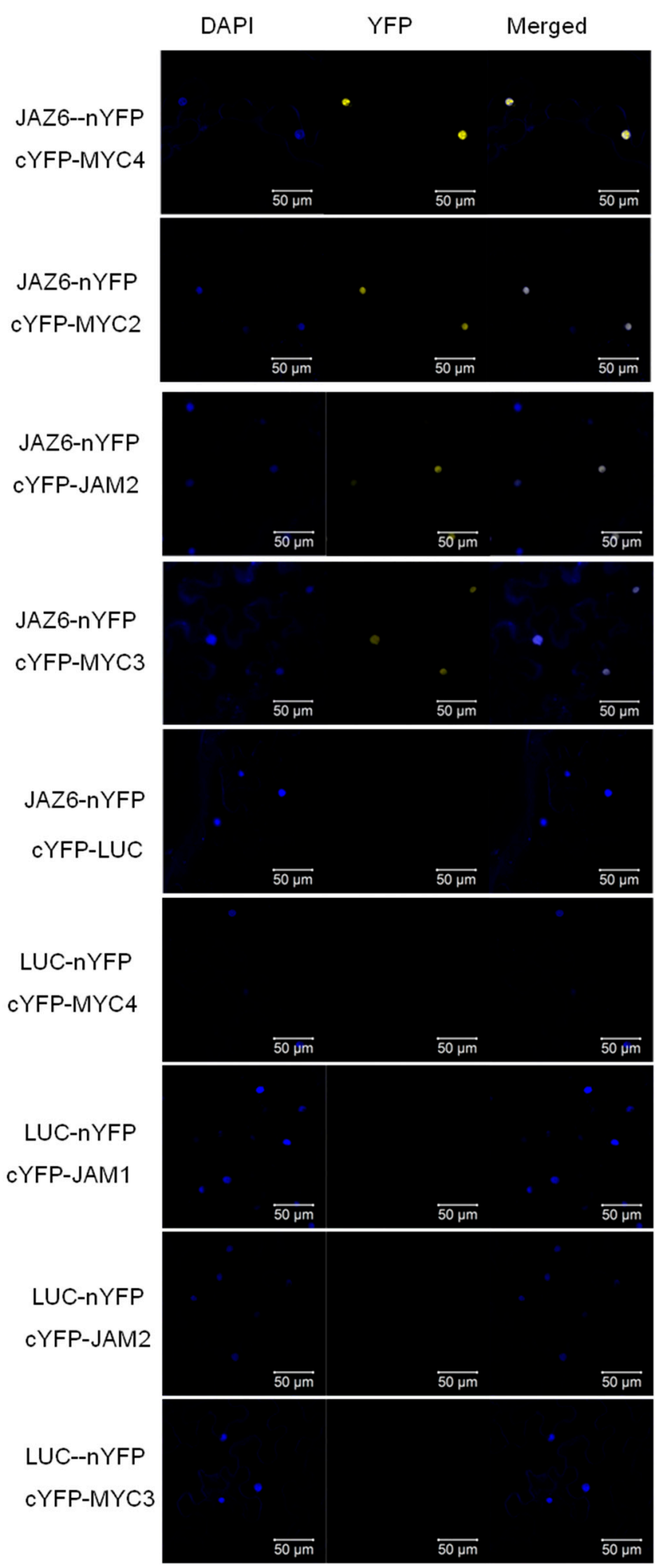

(b)

Figure 3. (a) yeast two-hybrid analysis of the interaction between JAZ and MYC2-like transcription factors. JAZ was ligated into pLexA and MYCs were ligated into pB42AD for yeast two-hybrid analysis.; (b) bimolecular fluorescence complementation analysis of the interaction between JAZ and MYCs.

Yeast two-hybrid results showed that transcription factors MYC2, MYC3 and MYC4 interact with most of the JAZ proteins. The exceptions with no interactions are present between MYC2 and JAZ1, MYC3 and JAZ1 and JAZ8, MYC4 and JAZ8. Like the predicted results, JAM1 lacking the JID domain 
has no interaction with 11 JAZs, but, unexpectedly, it can interact with JAZ8. Another inhibitory transcription factor, JAM2, interacts with JAZ4 JAZ12.

Bimolecular fluorescence complementation verified the interaction between JAZ6 and MYC2, MYC3, MYC4 and JAM2. JAZ also interacts with MYC type and JAM type transcription factors.

\subsubsection{JAZ and JAZ}

The interaction between AtJAZ and the others is an important condition for the transcription factor inhibition complex, and enriches the means of plant regulation of downstream pathway gene expression through the interaction between differently cleaved JAZ proteins.

The yeast two-hybrid assay was used in this study to analyse the interaction between JAZ in Taxus (Figure 4a).

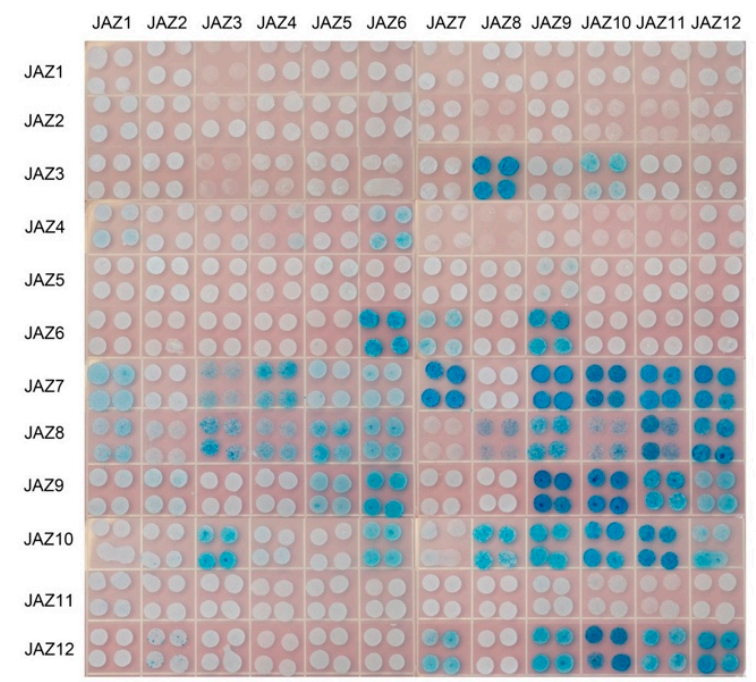

(a)

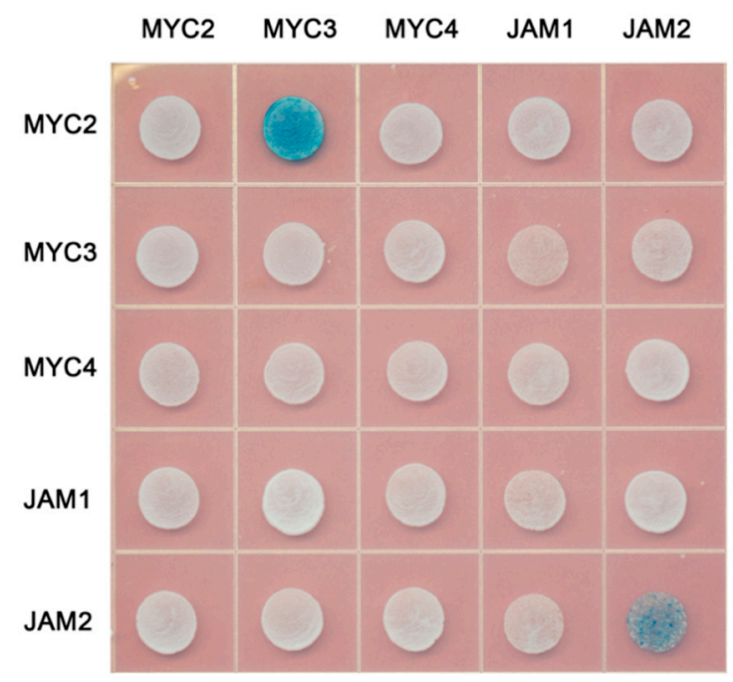

(b)

Figure 4. Yeast two-hybrid analysis (a) protein interactions between JAZ and JAZ; (b) protein interactions between MYC-type transcription factors.

Summarize the interaction between JAZ proteins as shown in Table 5 ("--"represents no signal, " +" represents a signal, the number of "+" represents the signal's strength):

Table 5. Yeast two-hybrid analysis of protein interactions between JAZ.

\begin{tabular}{|c|c|c|c|c|c|c|c|c|c|c|c|c|c|}
\hline & & \multicolumn{12}{|c|}{ pB42AD, Prey(AD) } \\
\hline & & JAZ1 & JAZ2 & JAZ3 & JAZ4 & JAZ5 & JAZ6 & JAZ7 & JAZ8 & JAZ9 & JAZ10 & JAZ11 & JAZ12 \\
\hline \multirow{12}{*}{$\begin{array}{l}\text { pLexA } \\
\text { Bait(BD) }\end{array}$} & JAZ1 & - & - & - & - & - & - & - & - & - & - & - & - \\
\hline & JAZ2 & - & - & - & - & - & - & - & - & - & - & - & - \\
\hline & JAZ3 & - & - & - & - & - & - & - & +++ & - & ++ & - & - \\
\hline & JAZ4 & + & - & - & + & - & ++ & - & - & - & - & - & - \\
\hline & JAZ5 & - & - & - & - & - & - & - & - & + & - & - & - \\
\hline & JAZ6 & - & - & - & - & - & +++ & ++ & - & ++ & - & - & - \\
\hline & JAZ7 & ++ & - & + & ++ & + & + & ++++ & - & +++ & +++ & +++ & +++ \\
\hline & JAZ8 & + & - & ++ & + & ++ & ++ & - & ++ & +++ & ++ & +++ & +++ \\
\hline & JAZ9 & - & + & - & - & ++ & ++ & - & - & ++++ & ++++ & +++ & +++ \\
\hline & JAZ10 & - & - & +++ & - & - & +++ & - & +++ & +++ & +++ & +++ & ++ \\
\hline & JAZ11 & - & - & - & - & - & - & - & - & - & - & - & - \\
\hline & JAZ12 & - & - & - & - & - & - & +++ & - & +++ & ++++ & +++ & +++ \\
\hline
\end{tabular}

\subsubsection{MYC and MYC}

The MYC transcription factor contains the domain of bHLH, in which the domain of HLH is responsible for the binding of MYC transcription factors to each other, forming a homologous or 
heterologous bimolecular structure in the plant to regulate the transcription of downstream genes. There is no interaction between activated MYC-like transcription factors and inhibitory MYC-like transcription factors in model plants. In this study, the interaction between MYC2, MYC3, MYC4 and JAM1, JAM2 of Taxus was analysed. The results are shown in Figure 4b.

Yeast two-hybrid results showed an interaction between MYC2 and MYC3, JAM2 interacted with itself, and there was no interaction between other MYC-type transcription factors.

\subsubsection{MYC and MED25}

The MYC-like transcription factor binds to MED25, thereby recruiting the RNA polymerase complex and activating transcription. Yeast two-hybrid study of the interaction between MYC2 and other transcription factors in Taxus and MED25; the results are shown in Figure 5a.

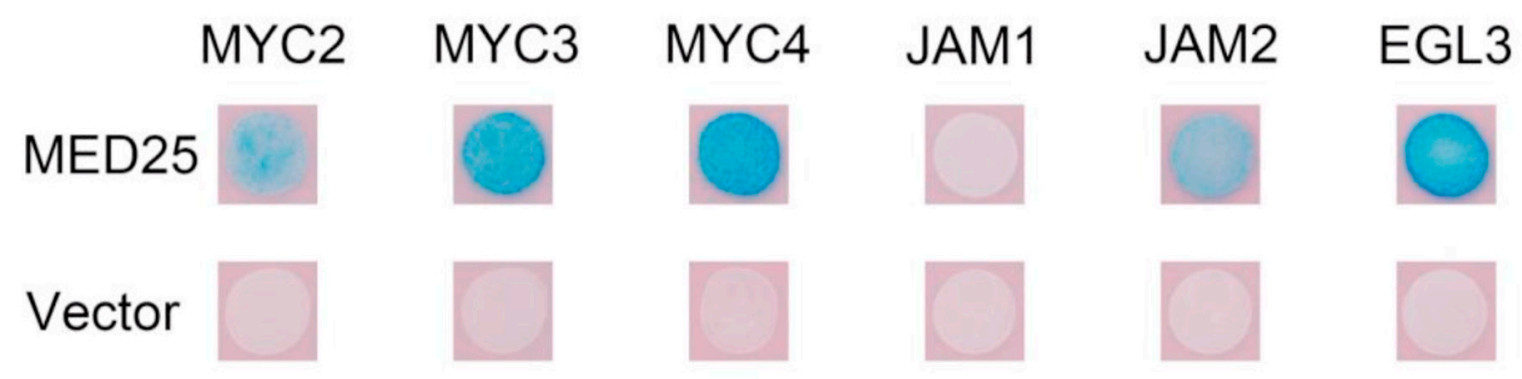

(a)

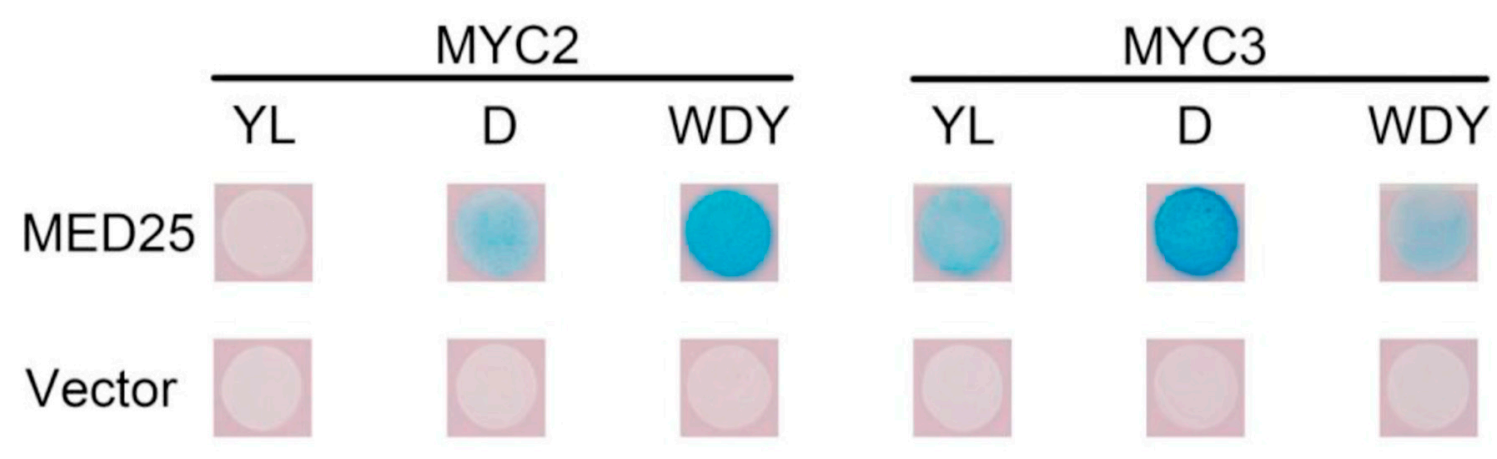

(b)

Figure 5. (a) Yeast two-hybrid analysis of protein interactions between MYC-type transcription factors and MED25; (b) results of yeast two-hybrid assay for mutated MYC-type transcription factors interacting with MED25.

The activated transcription factors MYC2, MYC3 and MYC4 in Taxus can bind to MED25, while JAM1 has no interaction with MED25. Surprisingly, JAM2 binds to MED25, indicating that it may have some transcriptional activation activity. EGL3, which may be involved in anthocyanin synthesis, may also bind to MED25, indicating that it may also have transcriptional activation activity.

\subsection{Jasmonic Acid Signalling Pathway MYC Transcription Factor Regulates Paclitaxel Biosynthesis}

\subsubsection{Detection and Analysis of MYC Transcription Factor Activity}

To further confirm the activity of the MYC-type transcription factor, the transcriptional activity of the relevant transcription factors was verified in Arabidopsis protoplasts (see Figure A4). GAL4BD, which binds to the GAL4 promoter, was fused to a transcription factor and the transcriptional activities of MYC2, MYC3, MYC4, JAM1 and JAM2 in protoplasts were examined. 
Among them, the positive control AtMYB21 had strong transcriptional activation activity, and the negative control AtbHLH17 had no transcriptional activation activity. The transcription factor MYC2 in Taxus had strong transcriptional activation, and the transcriptional activation activities of MYC3 and MYC4 were low. While JAM1 and JAM2 had almost no activation activity, the results of yeast two-hybridization are consistent with the fact that JAM2 is more active than JAM1.

\subsubsection{Synthetic Pathway Gene Promoter Separation and Analysis}

To analyse the regulation of transcription factor on the expression of paclitaxel synthesis pathway gene, it is important to isolate the promoter of the synthesis pathway gene. In the existing literature, only a few promoters of the paclitaxel synthesis pathway have been isolated but they were not long enough for the analysis of the promoter properties $[2,10]$.

Therefore, a Genome walker PCR was used to isolate the promoters of all 14 known genes in the Taxol synthesis pathway in Taxus. The sequence is shown in the Appendices A and B. To investigate the possible regulation of these genes by MYC transcription factors, we analysed the number of E-box (CANNTG) that can bind to MYC transcription factors, especially the G-box which has the strongest binding ability to MYC transcription factors. CACGTG sequences and variant G-boxes (AACGTG, CACGAG and special E-box, CATGTG CATGTG) are defined as High Affinity (HA) E-box; AACGTG and CACGAG are defined as modified G-box, and other E-boxes are defined as low affinity (LA) E-box [15]. The number of promoters in the gene was analysed, and the promoter information of the obtained gene is shown in Table 6:

Table 6. List of cis-element analysis information in the paclitaxel biosynthesis pathway gene promoter sequence.

\begin{tabular}{cccccccc}
\hline $\begin{array}{c}\text { Gene } \\
\text { Name }\end{array}$ & $\begin{array}{c}\text { Promoter } \\
\text { Length }\end{array}$ & G-Box & $\begin{array}{c}\text { Modified } \\
\text { G-Box }\end{array}$ & HA E-Box & $\begin{array}{c}\text { Total HA } \\
\text { cis-Element }\end{array}$ & LA E-box & Folds at $3 \mathbf{h}$ \\
\hline TBT & 1378 & 0 & 2 & 7 & 9 & 5 & 94.1 \\
TAT & 1153 & 1 & 4 & 2 & 7 & 4 & 39.9 \\
DBTNBT & 1257 & 1 & 2 & 2 & 5 & 4 & 9.9 \\
$2 \alpha \mathrm{OH}$ & 1179 & 1 & 0 & 3 & 4 & 3 & 16464.7 \\
$7 \beta \mathrm{OH}$ & 1481 & 0 & 0 & 4 & 4 & 2 & 748.5 \\
$10 \beta \mathrm{OH}$ & 1144 & 0 & 1 & 3 & 4 & 4 & 14.8 \\
PAM & 501 & 1 & 1 & 2 & 4 & 2 & 656.5 \\
TS & 1183 & 1 & 3 & 0 & 4 & 5 & 16.3 \\
$5 \alpha \mathrm{OH}$ & 1002 & 2 & 0 & 1 & 3 & 3 & 33.3 \\
$13 \alpha \mathrm{OH}$ & 734 & 0 & 2 & 1 & 3 & 3 & 3155.3 \\
DBAT & 1820 & 0 & 1 & 1 & 2 & 6 & 48 \\
BAPT & 976 & 0 & 1 & 0 & 1 & 2 & 19.5 \\
GGPPS & 970 & 0 & 1 & 0 & 1 & 6 & 3.5 \\
$14 \beta \mathrm{OH}$ & 792 & 0 & 0 & 0 & 0 & 1 & 33 \\
\hline
\end{tabular}

The 14 promoters of th synthetic pathway genes all contain E-box, and all of them are induced by jasmonic acid, and the promoters of six genes contain G-box. The TBT promoter contains the most E-box and the most cis-elements with high affinity for MYC-like transcription factors. The $14 \beta \mathrm{OH}$ promoter contains the least amount of E-box and is low-affinity. The specific location of the cis component is shown in Figure A5.

\subsubsection{Activated MYC Transcription Factor Induces a Paclitaxel Biosynthesis Pathway Gene Promoter}

Gel electrophoresis migration experiments demonstrated that full-length MYC2 binds to G-box and high-affinity E-box [2]. This indicates that MYC transcription factors have the potential to activate paclitaxel synthesis pathway gene expression. To study the regulation of MYC transcription factors on metabolic pathway genes in this study, plasmids with fluorescent protein and related gene promoter that continuously express transcription factors were transfected into Arabidopsis protoplasts. 
The regulation of MYC2, MYC3 and MYC4 on the promoter of paclitaxel synthesis pathway gene was examined. The principle is shown in Figure A6.

For each promoter, the fluorescence intensity data ratio was normalized to the 62 sk plasmid as a reference, and the results were as Figure 6:

TBT

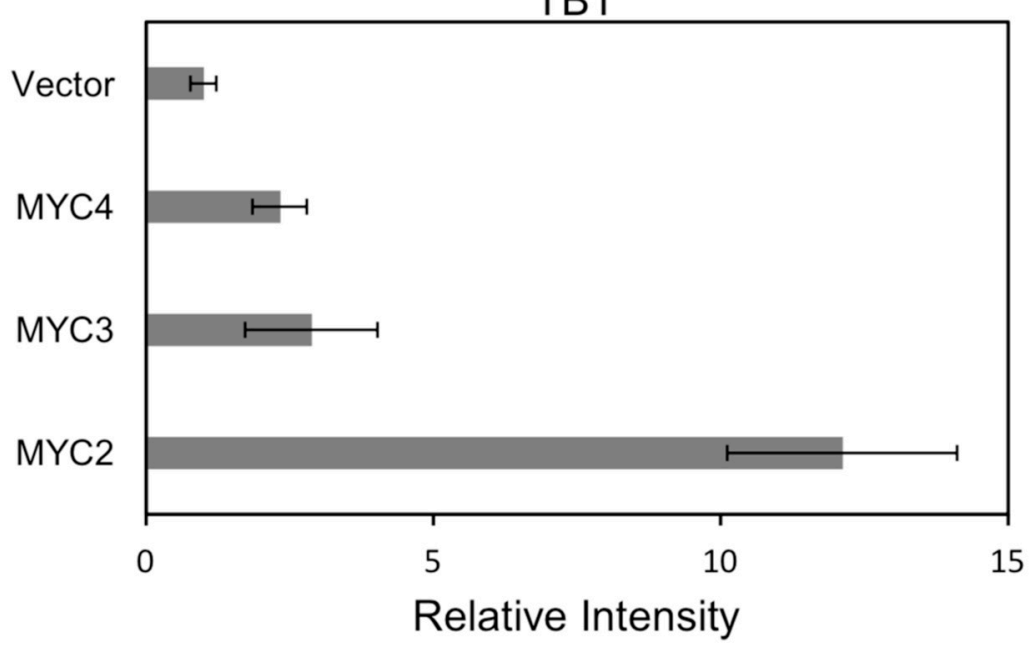

TAT

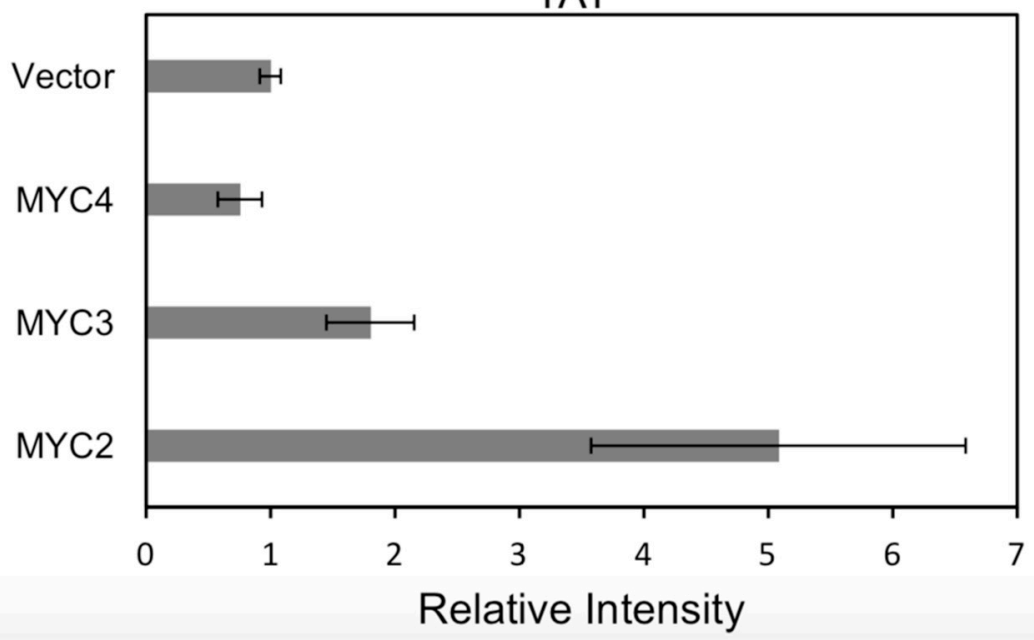

(a)

Figure 6. Cont. 

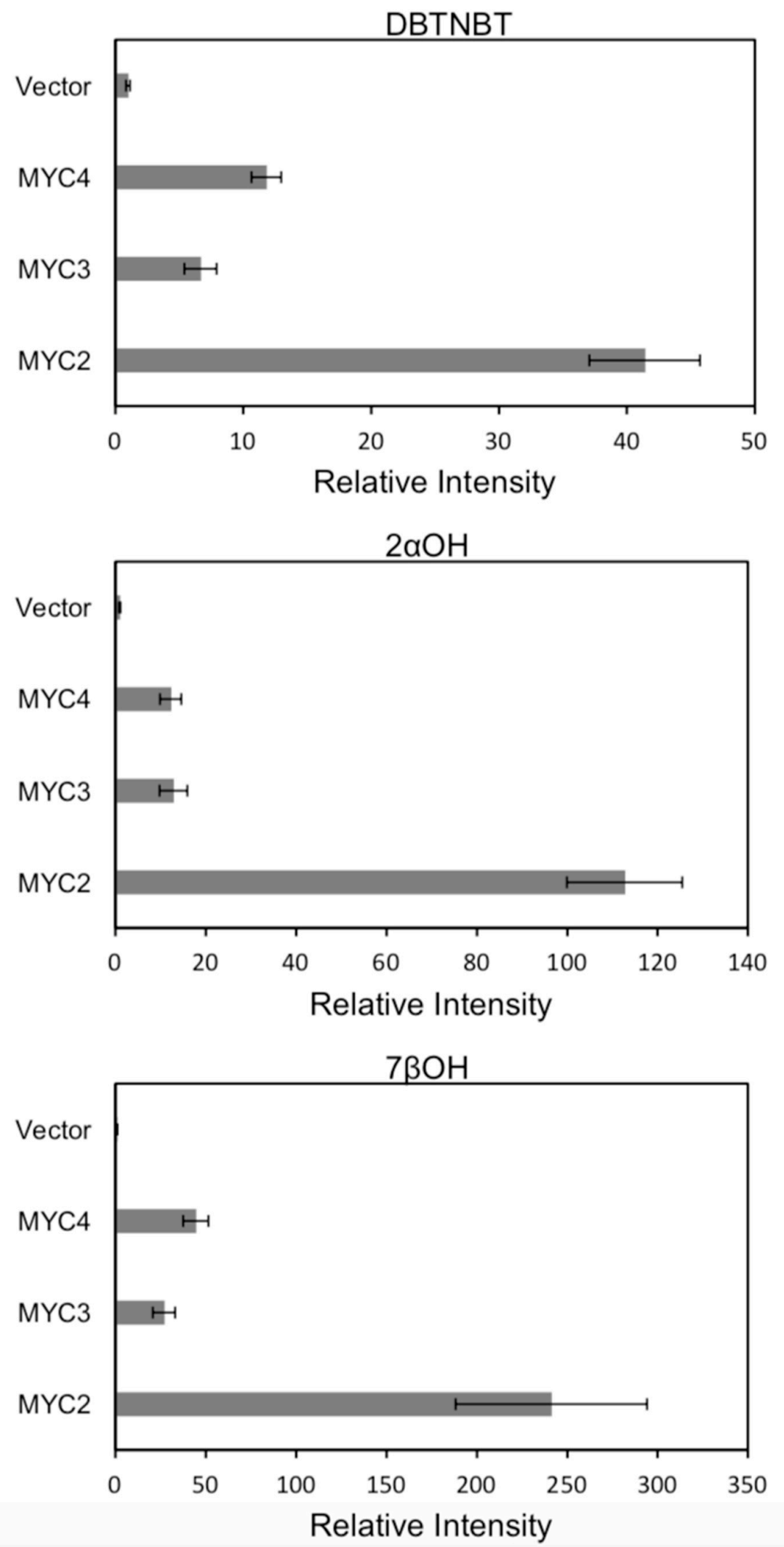

(b)

Figure 6. Cont. 

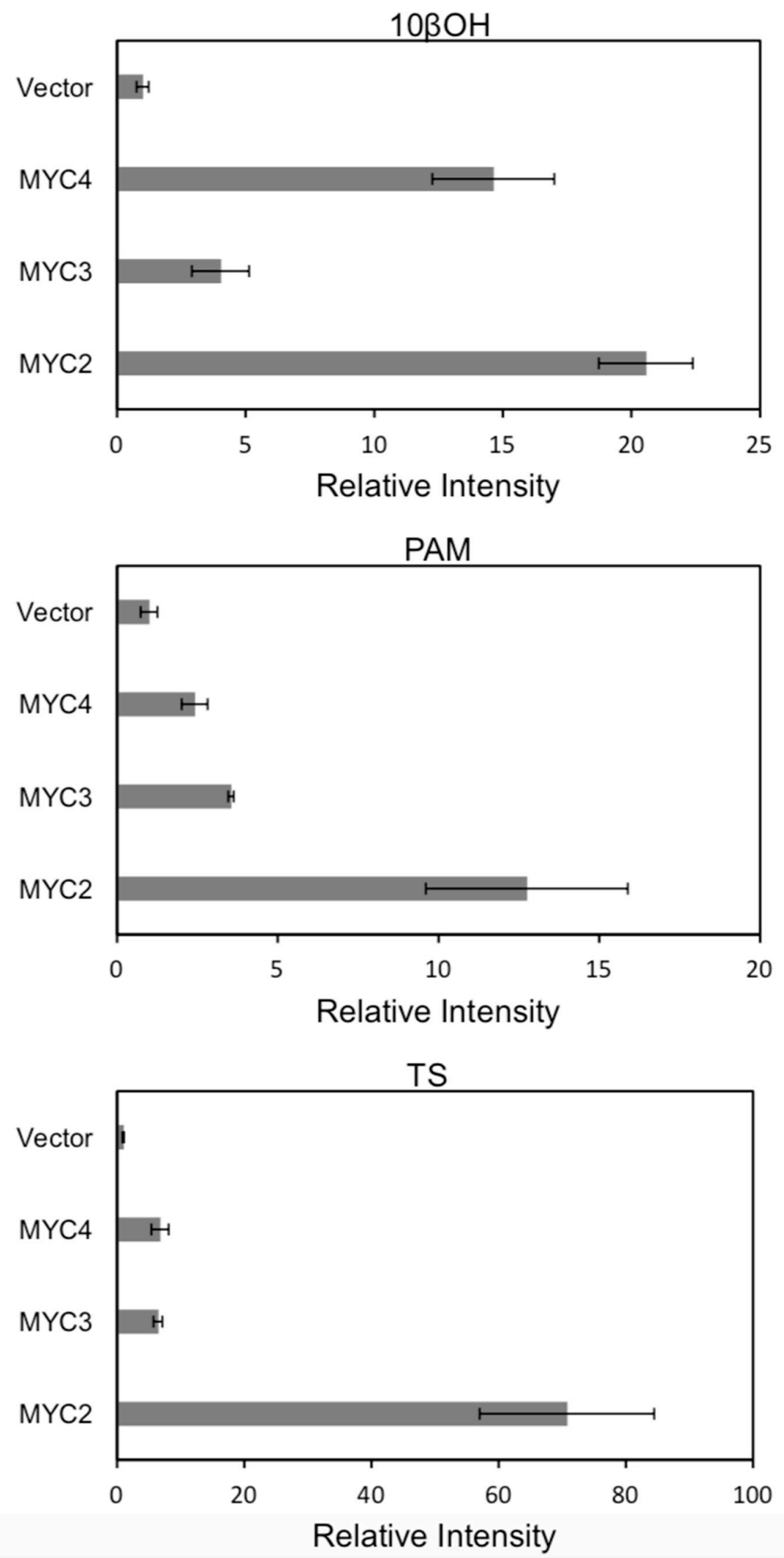

(c)

Figure 6. Cont. 

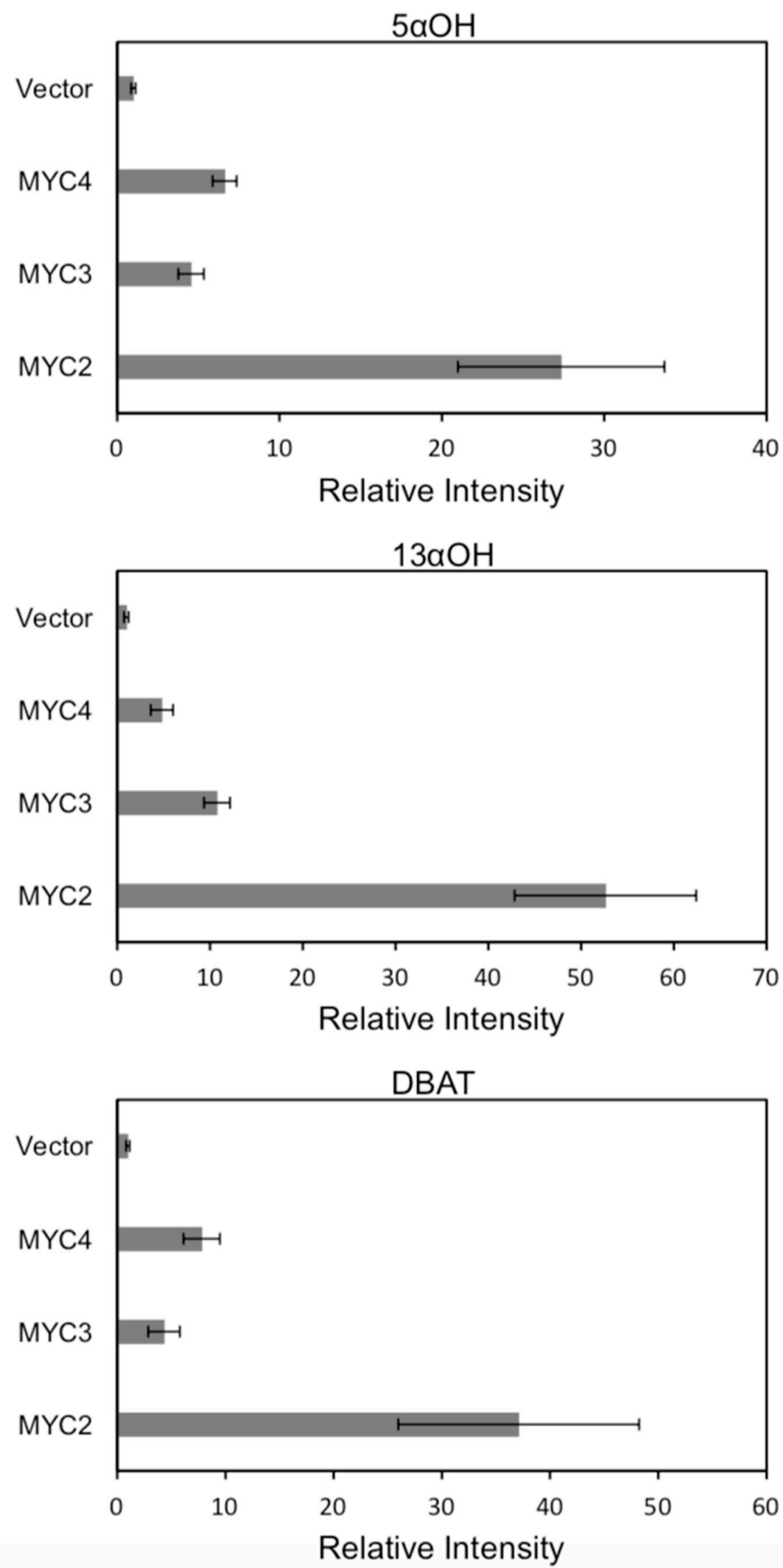

(d)

Figure 6. Cont. 

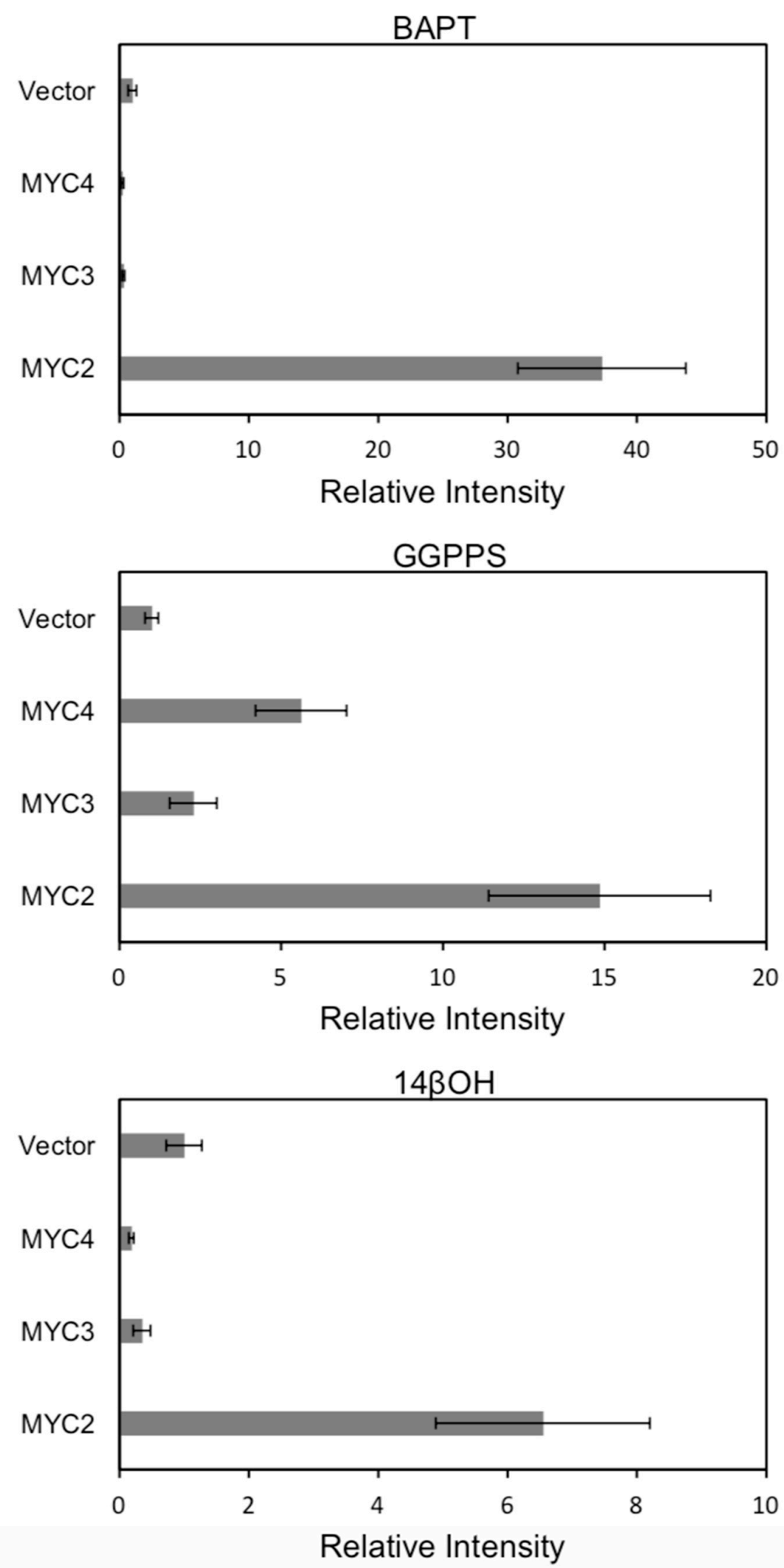

(e)

Figure 6. (a) transcriptional activity of MYC2, MYC3 and MYC4 on the paclitaxel synthesis pathway genes TAT and TBT promoters (Results are presented as a LUC/REN ratio. $n=3$ in all samples, error bars are SE(TBT group, ${ }^{*} p=3.26 \times 10^{-6}$; TAT group, $\left.{ }^{*} p=7.26 \times 10^{-6}\right) ;(\mathbf{b})$ transcriptional activity of MYC2, 
MYC3 and MYC4 on the paclitaxel synthesis pathway genes DBTNBT, $2 \alpha \mathrm{OH}$ and $7 \beta \mathrm{OH}$ promoters (Results are presented as a LUC/REN ratio. $n=3$ in all samples, error bars are SE(DBTNBT group, ${ }^{*} p=9.19 \times 10^{-8} ; 2 \alpha \mathrm{OH}$ group, ${ }^{*} p=3.02 \times 10^{-7} ; 7 \beta \mathrm{OH}$ group, $\left.{ }^{*} p=4.95 \times 10^{-8}\right)$; (c) transcriptional activities of MYC2, MYC3 and MYC4 on the paclitaxel synthesis pathway genes $10 \beta \mathrm{OH}$, PAM and TS promoters (Results are presented as a LUC/REN ratio; $n=3$ in all samples, error bars are SE $(10 \beta \mathrm{OH}$ group, ${ }^{*} p=3.86 \times 10^{-4}$; PAM group, ${ }^{*} p=0.0264$; TS group, $\left.{ }^{*} p=0.00528\right) ;(d)$ transcriptional activities of MYC2, MYC3 and MYC4 on the paclitaxel synthesis pathway genes $5 \alpha \mathrm{OH}, 13 \alpha \mathrm{OH}$ and DBAT promoters (Results are presented as a LUC/REN ratio. $n=3$ in all samples, error bars are $\mathrm{SE}(5 \alpha \mathrm{OH}$ group, ${ }^{*} p=3.21 \times 10^{-6} ; 13 \alpha \mathrm{OH}$ group, ${ }^{*} p=4.67 \times 10^{-11}$; DBAT group, $\left.{ }^{*} p=0.00359\right)$; (e) transcriptional activity of MYC2, MYC3 and MYC4 on paclitaxel synthesis pathway genes BAPT, GGPPS and $14 \beta \mathrm{OH}$ promoters (Results are presented as a LUC/REN ratio. $n=3$ in all samples, error bars are SE(BAPT group, ${ }^{*} p=1.31 \times 10^{-6} ;$ GGPPS group, ${ }^{*} p=3.37 \times 10^{-5} ; 14 \beta \mathrm{OH}$ group, ${ }^{*} p=3.72 \times 10^{-7}$ ).

The activation of the same transcription factor for the promoters of different genes is shown in Figure 7a-c:

\section{MYC2}

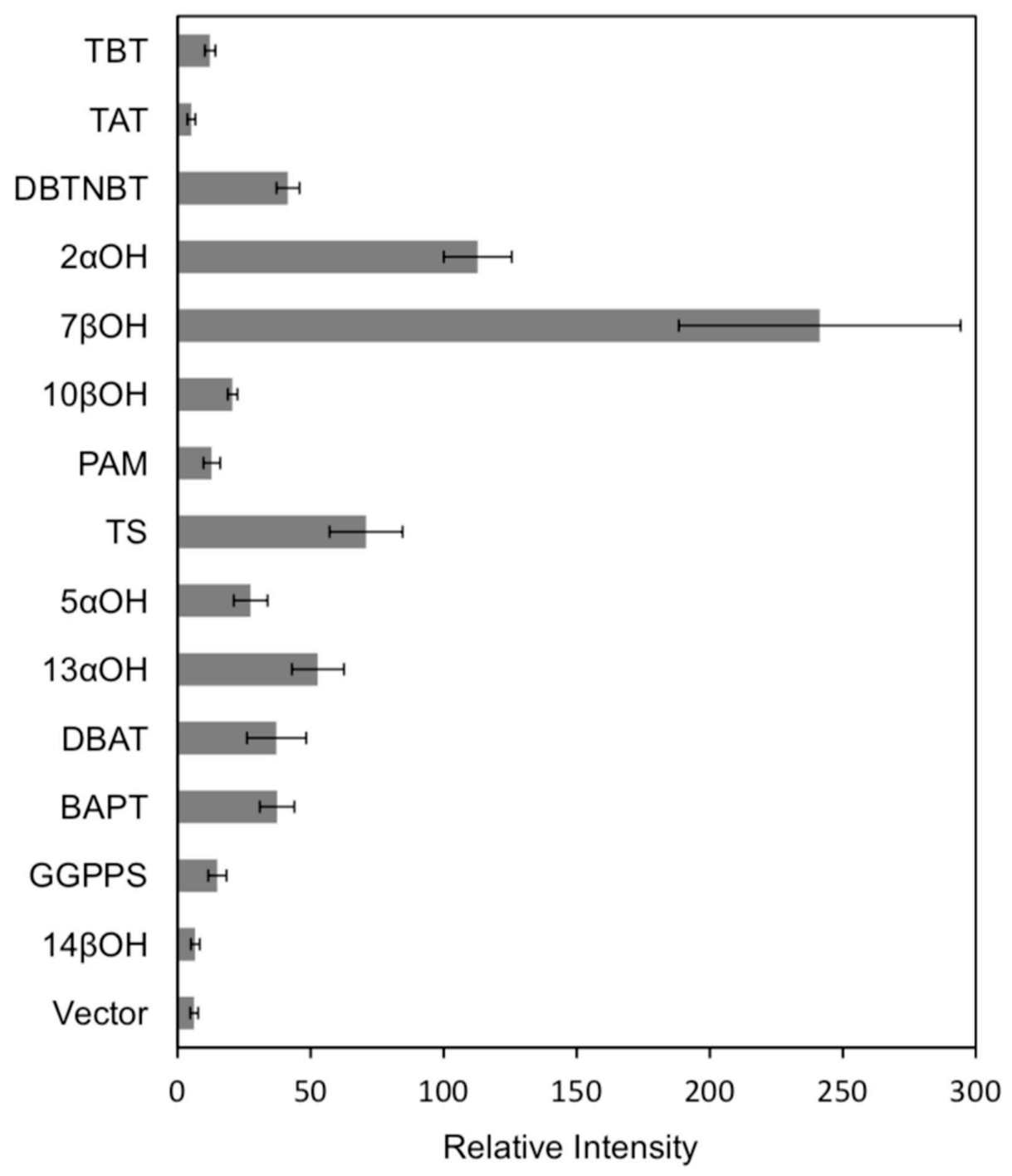

(a)

Figure 7. Cont. 


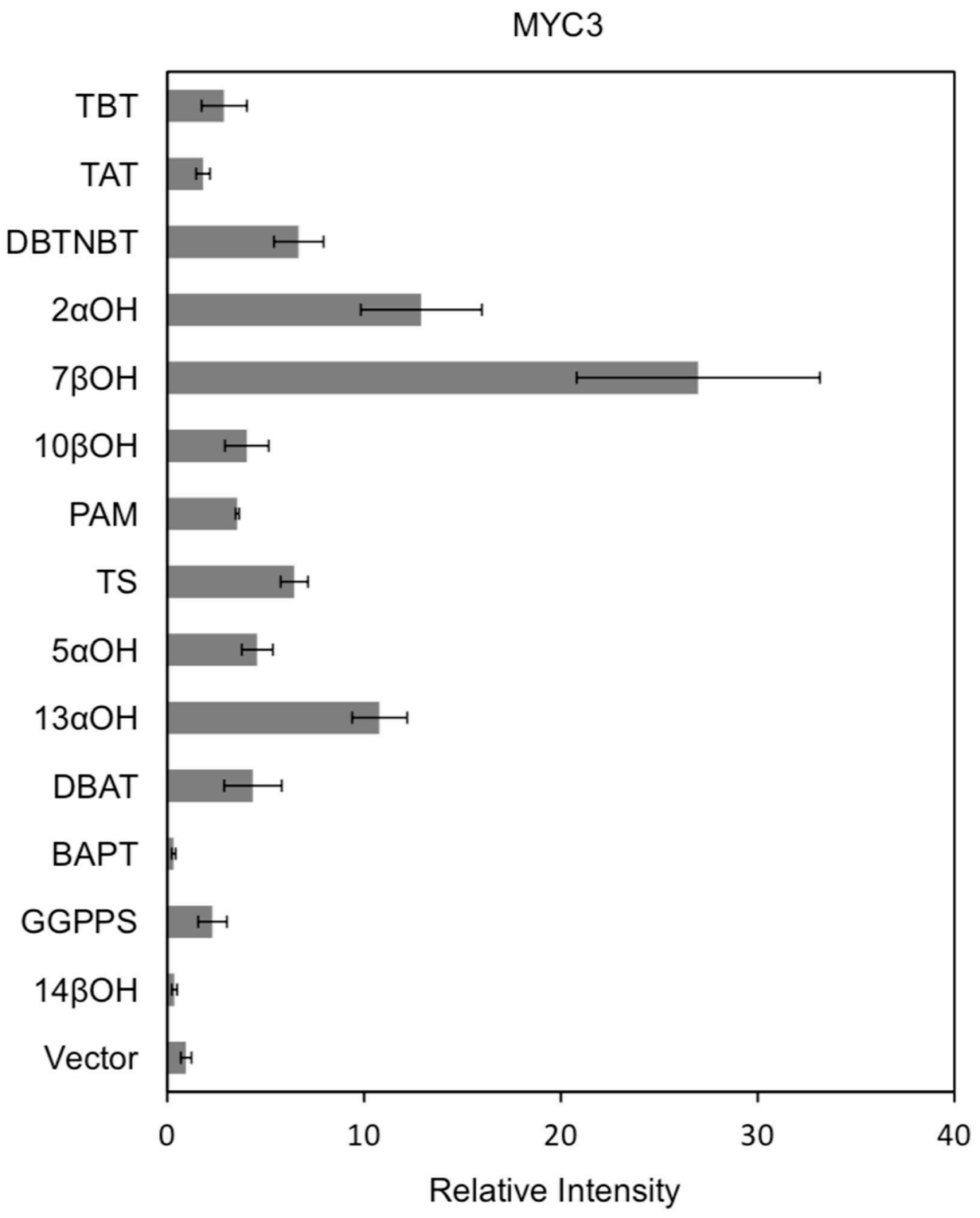

(b)

Figure 7. Cont. 
MYC4

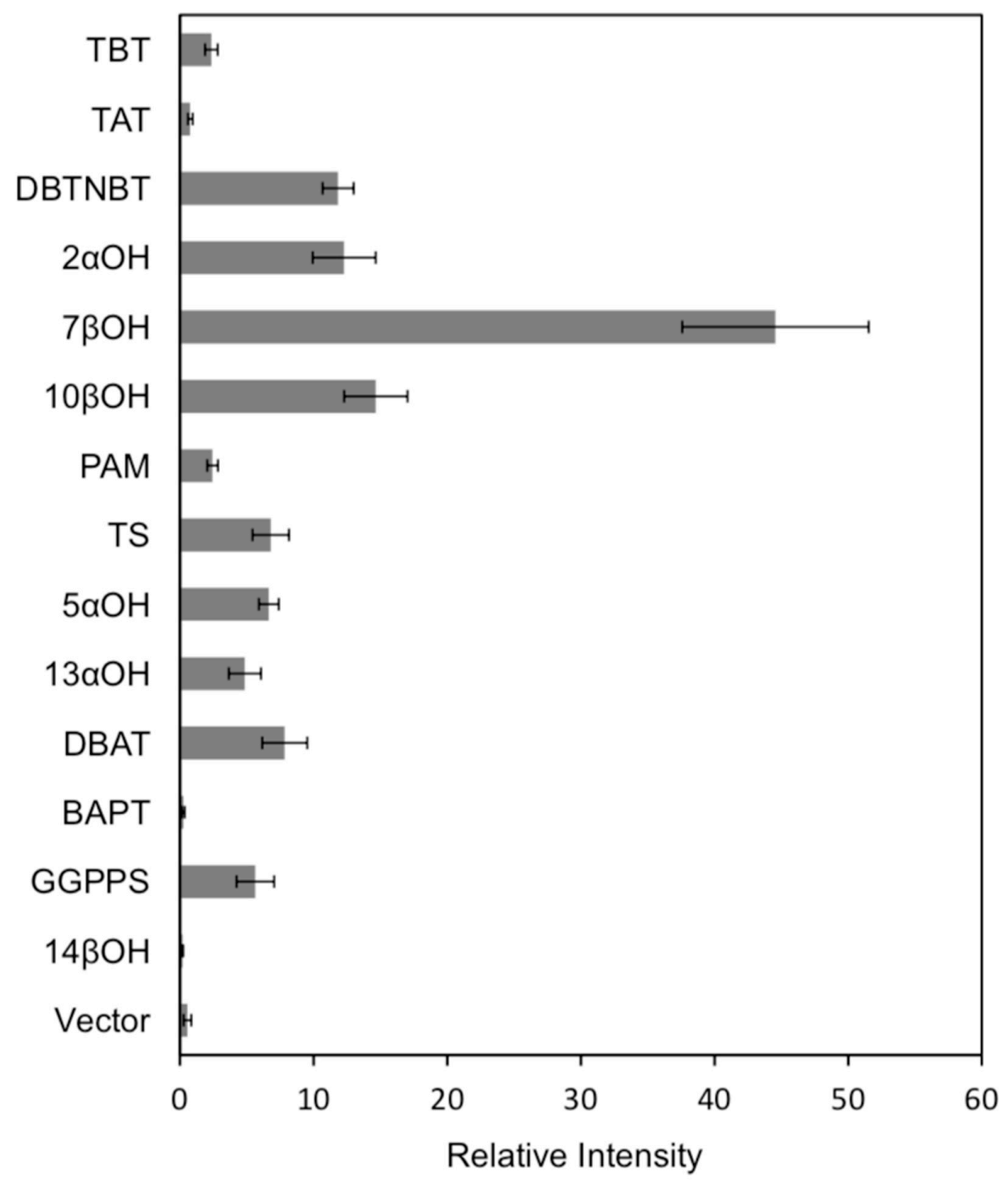

(c)

Figure 7. Cont. 


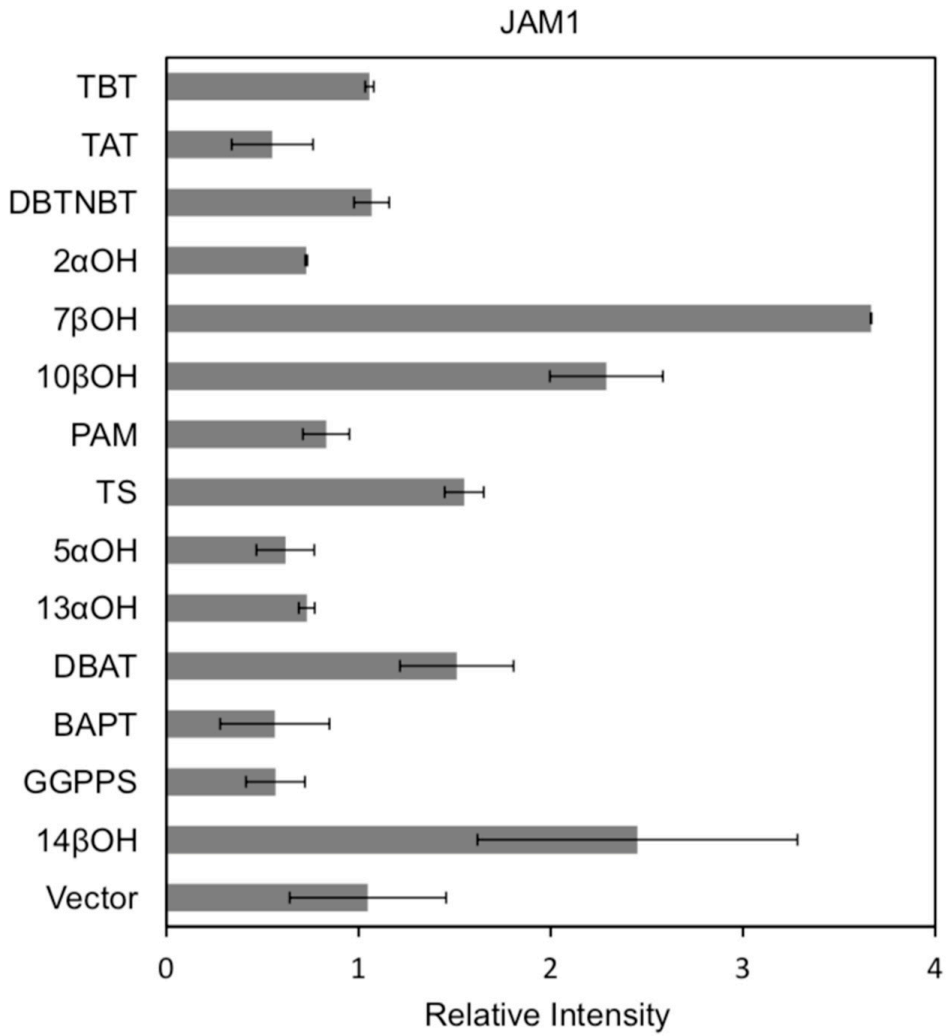

(d)

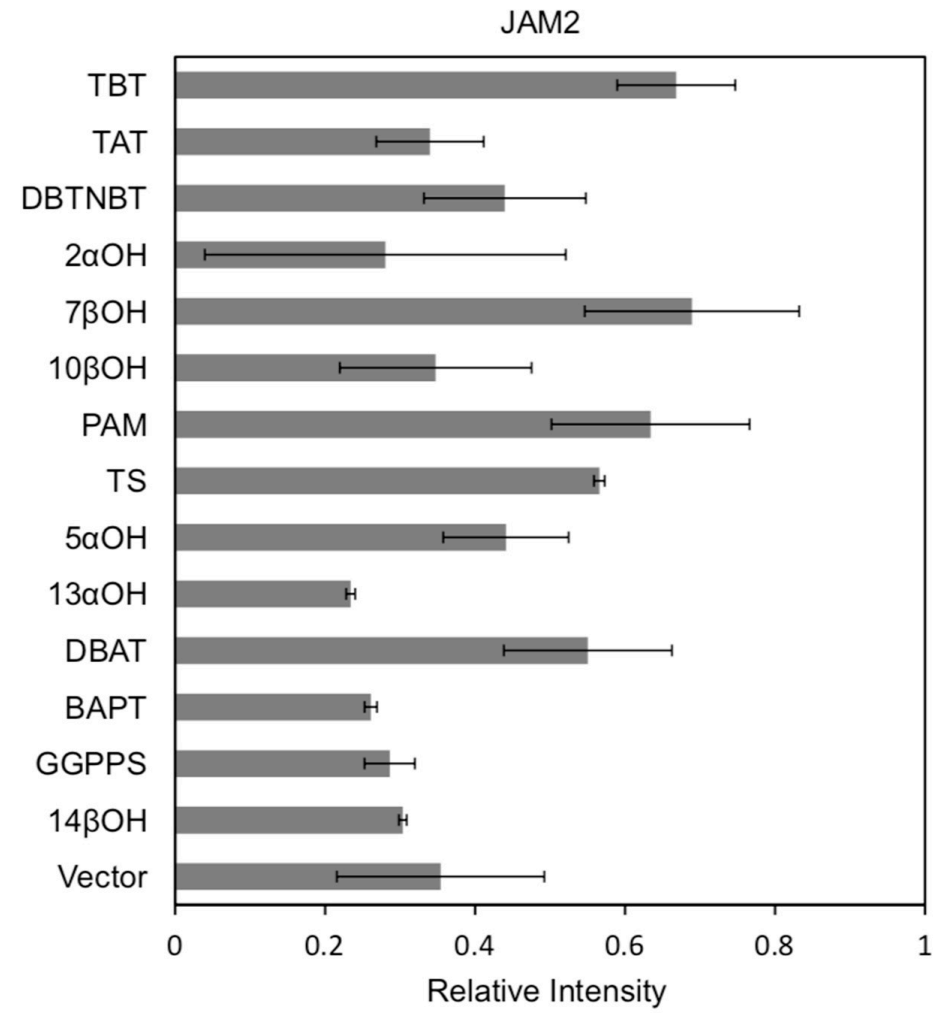

(e)

Figure 7. (a) transcriptional activity of MYC2 on the promoters of 14 genes known for the paclitaxel synthesis pathway (Results are presented as a LUC/REN ratio. $n=3$ in all samples, error bars are SE $*^{*} p$ $\left.=2 \times 10^{-5}\right)$; (b) transcriptional activity of MYC 3 on the promoters of 14 genes with known paclitaxel 
synthesis pathways (Results are presented as a LUC/REN ratio. $n=3$ in all samples, error bars are SE $\left({ }^{*} p=1.15 \times 10^{-11}\right)$; (c) MYC4 pairs Transcriptional activity of promoters of 14 genes known for the paclitaxel synthesis pathway (Results are presented as a LUC/REN ratio. $n=3$ in all samples, error bars are $\mathrm{SE}\left({ }^{*} p=2.43 \times 10^{-6}\right)$; (d) transcriptional activity of the promoter of the paclitaxel synthesis pathway partial gene (Results are presented as a LUC/REN ratio. $n=3$ in all samples, error bars are SE $\left.{ }^{*} p=1.7 \times 10^{-7}\right)$; (e) transcriptional activity of the promoter of the paclitaxel synthesis pathway partial gene (Results are presented as a LUC/REN ratio. $n=3$ in all samples, error bars are SE $\left({ }^{*} p=1.71 \times 10^{-7}\right)$.

In comparison, among the three activating transcription factors of the jasmonic acid signalling pathway, MYC2, MYC3, and MYC4, although they have different effects on each promoter, MYC2 can activate the expression of all promoter-induced genes. MYC3 and MYC4 had no induction effect on the promoter of BAPT and $14 \beta \mathrm{OH}$, and both had weaker activation effects on the promoters of TAT and TBT.

The promoters of the genes for the paclitaxel synthesis pathway are substantially similar in relative strength induced by the above transcription factors. The classification is as follows: the promoter of DBTNBT, $2 \alpha \mathrm{OH}, 7 \beta \mathrm{OH}$, TS is the strongest; followed by $5 \alpha \mathrm{OH}, 13 \alpha \mathrm{OH}$, DBAT; followed by PAM, $10 \beta \mathrm{OH}$, GGPPS; while the promoters of TAT and TBT and BAPT, $14 \beta \mathrm{OH}$ are the weakest. For the promoters of the latter two genes, two of them have an effect of inhibiting transcription.

\subsubsection{Inhibitory MYC Transcription Factor Inhibits Activation MYC Transcription Factor}

The model plant Arabidopsis has four inhibitory MYC transcription factors that bind to the promoter by competitively binding to the activated MYC transcription factor, thereby inhibiting the action of the activated MYC transcription factor [16,17]. For JAM1 and JAM2 isolated from Taxus, it was found that there was no corresponding transcriptional activation in protoplasts. The result is shown in Figure $7 \mathrm{~d}$,e. In the future, JAM1 and JAM2 overexpression plasmid and reporter genes regulated by all gene promoters should be transferred into protoplasts, and further investigated for it can activate the transcription of the promoter of paclitaxel synthesis pathway gene.

JAM1 and JAM2 had no significant transcriptional activation of all of the tested promoters. Yeast two-hybrid results showed that the inhibitory MYC transcription factor could not bind to the activated MYC transcription factor, similar to that in the model plant. Whether or not it is similar to the model plant, it inhibits the action of the activated transcription factor by competitively binding the promoter sequence to the activated MYC-like transcription factor.

Subsequent studies will co-transfect plasmids expressing inhibitory and activating transcription factors in protoplasts, investigate the strength of transcriptional activity, and analyse whether they have similar inhibitory effects and principles.

\subsubsection{Mutant MYC Transcription Factor Activity is not Affected by JAZ}

JAZ is an important protein in the signal reception process of jasmonic acid, and it is also a protein that inhibits the activity of transcription factors, and it is also induced by jasmonic acid signal, which plays a feedback inhibition effect in the jasmonic acid signalling pathway. A mutant MYC-like transcription factor in a model plant that does not interact with JAZ but activates transcription of a downstream pathway gene, providing an idea for using biotechnology to increase the signalling pathway of jasmonic acid.

In Taxus, JAZ also inhibits the expression of downstream pathway genes by binding to activated transcription factors. This feedback inhibition is an important means by attenuating the effects of JAZ to attenuate the jasmonic acid signaling pathway. However, the number of JAZ in Taxus is high, and the way of mutating JAZ to regulate downstream signalling pathways is complicated, and mutations and their interacting MYC transcription factors are feasible methods.

By analysing the complex structure of AtJAZ and AtMYC3 in model plants, the MYC2 and MYC3 variants were designed by MYC2YL, MYC2D, MYC2WDY and MYC3YL, which are the conserved 
amino acids that play an important role in the binding of JAZ-like transcription factors to JAZ. MYC3D and MYC3WDY (for detailed mutation sites, refer to the sequence of each transcription factor variant in the Appendices A and B).

First, the binding of the above six MYC transcription factor variants to MED25 was analysed by yeast two-hybrid. The result is shown in Figure 5b. From comparison of results from the unmutated MYC experimental group, it can be seen that, apart from MYC2YL which does not bind to MED25, other mutations do not affect the binding of MYC transcription factors to MED25, indicating that the transcriptional activities of other mutant MYC transcription factors are likely to be unaffected.

The interaction between $12 \mathrm{JAZ}$ and mutant MYC was further studied by yeast two-hybrid assay to analyse whether the mutant MYC could bind to JAZ. The result is shown in Figure 8.

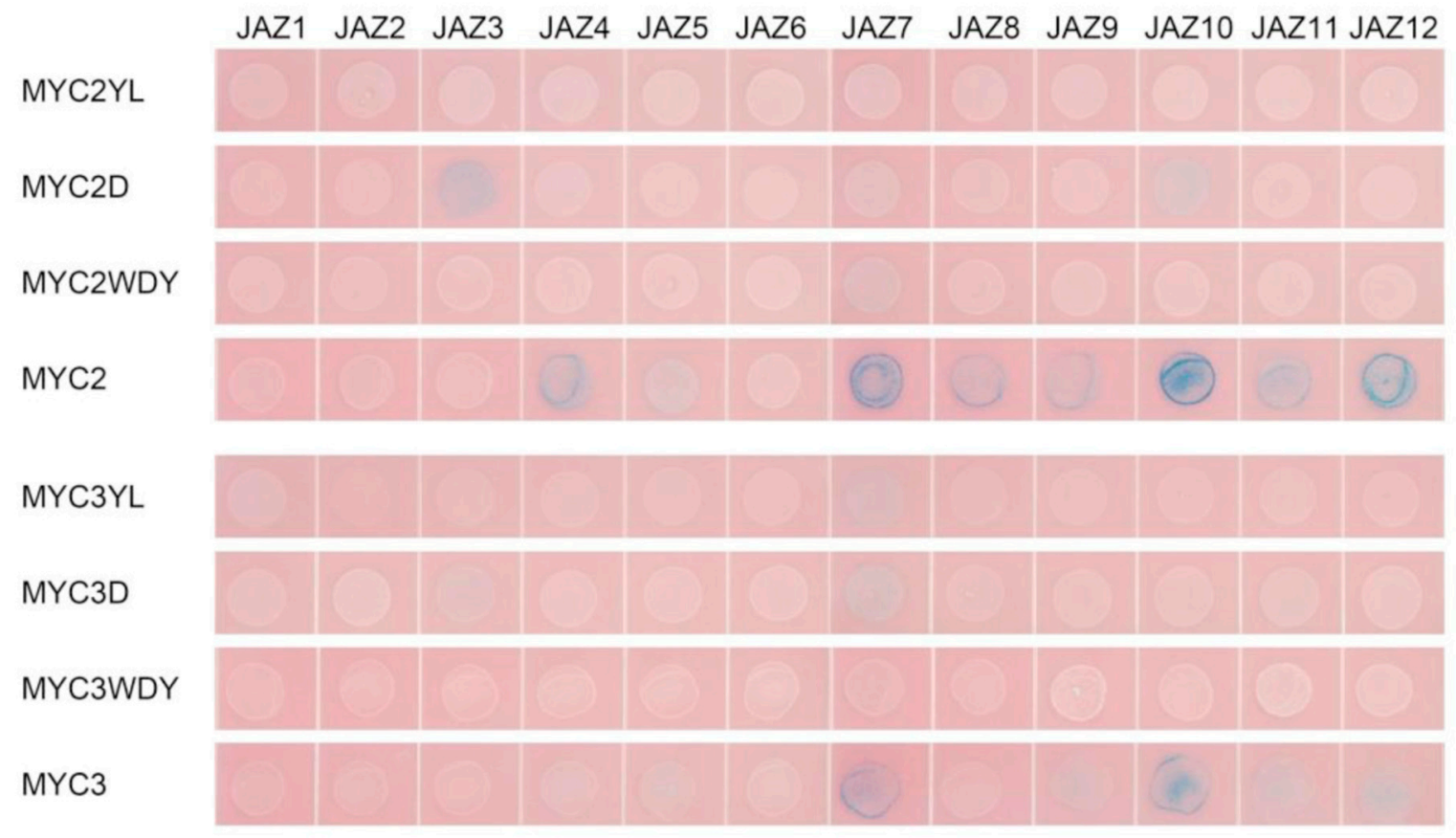

Figure 8. Results of the interaction between the MYC-type transcription factor and JAZ in a yeast two-hybrid assay.

Three forms of mutations all affect the protein interaction between JAZ and MYC transcription factors. Mutation of a single D amino acid site affects the binding of MYC2 and MYC3 to most JAZ, while the mutation of three amino acids of WDY almost prevents MYC2 and MYC3 from binding to all JAZ proteins. The mutation of two amino acids of YL has a stronger effect in MYC2, which makes MYC2 unable to bind to all JAZ, but also cannot bind to MED25; the YL mutation of MYC3 makes it have no interaction with any JAZ except JAZ7.

In summary, the WDY mutation of MYC2 and the YL mutation of MYC3 may be novel transcription factors that are not inhibited by JAZ but have strong transcriptional activity and can be used to improve gene expression of paclitaxel biosynthesis pathway.

The regulation of the MYC-like transcription factor of the mutant on the paclitaxel biosynthetic pathway promoter will be further verified in protoplasts.

\section{Discussion}

\subsection{Taxus Has a Similar Mechanism of Signal Accepting and Conduction of Jasmonic Acid}

As an important phytohormone, jasmonic acid is an important component of plant stress response. It has been proven to be ubiquitous in plants, not only in the model plant Arabidopsis thaliana [14,18-21]. 
Similar acceptance and transmission pathways for jasmonic acid signals should also be present in the Taxus. Prior to this study, a small number of genes for the jasmonic acid signalling pathway have been isolated by high-throughput sequencing [10], but have not conducted in-depth studies.

The jasmonic acid signalling pathway in Taxus was confirmed by high-throughput sequencing and identification of related genes and their function. Twenty-one isolated genes of the jasmonic acid signalling pathways were similar to those found in the model plants, considering characteristic protein domains and protein-protein interactions. It is shown that a similar jasmonic acid signalling pathway may exist in Taxus and play an important role.

Firstly, 12 JAZ interacted with two COI1 in the presence of coronatine, and the interaction intensity of some JAZ and COI1 increased with the increase of coronin concentration, indicating that these are probably active molecules of jasmonic acid signalling in Taxus. Jasmonyl isoleucine also mediates the interaction between the JAZ and COI1. However, JAZ8 and JAZ9 also have strong interaction with COI1 in the absence of coronatine, which is different from that in model plants. This indicates that the signalling pathways in Taxus may be different. The relevant biological significance requires in-depth research.

There is also an interaction between the protein of MYC transcription factor and the JAZ protein in Taxus, which is an important way for JAZ to inhibit the transcriptional activities of MYC2, MYC3 and MYC4. JAM1, lacking the JID domain, does not bind to most JAZ, indicating that the mutual binding is based on the JID domain in the MYC-like transcription factor. JAZ8, which contains two EAR domains and has a long length and JAS domain, is not combined with MYC3 and MYC4, but can be combined with MYC2 and the JAM1 without the JID domain. It may play a special role in the jasmonic acid signalling pathway of Taxus. Subsequent structural analysis of the complexes formed by AtMYC 3 and AtJAZ1 of A. thaliana resulted in mutations in MYC2 and MYC3, and mutations in the JID domain resulted in their inability to bind to JAZ. This also suggests that the jasmonic acid signalling pathway in Taxus is similar to that in Arabidopsis [16,22-24]. Interactions exist between JAZ proteins, especially between any combinations of any two of the six JAZs from JAZ7 to JAZ12, but there is less interaction between any combinations of any two of the six JAZs from JAZ1 to JAZ6, indicating that JAZ7-JAZ12 plays an important role in the formation of inhibitory protein complexes [22].

There are also interactions between MYC transcription factors, MYC2 and MYC3, and JAM2 interacts with itself. This differs from the broad interaction between inhibitory MYC transcription factors between activated MYC-like transcription factors in model plants.

The final MYC-type transcription factor recruits RNA polymerase by binding to MED25 to activate transcription of downstream genes. MYC2, MYC3 and MYC4 in Taxus have the same function. JAM1 without activation activity could not bind to MED25. However, JAM2, which has no apparent transcriptional activity, binds to MED25, indicating that it may bind to MED25 competitively, thereby inhibiting jasmonic acid signalling.

\subsection{Jasmonic Acid Signalling Pathway Transcription Factor Regulates Paclitaxel Biosynthesis Pathway Gene Expression}

The transcription factors MYC2, MYC3 and MYC4 of the jasmonic acid signalling pathway play an important role in the paclitaxel biosynthesis pathway. In the promoter region of most paclitaxel synthesis pathway genes, there are G-boxes and similar cis-elements that bind to MYC-type transcription factors, including the promoters of TAT, TBT, DBTNBT, $2 \alpha \mathrm{OH}, 7 \beta \mathrm{OH}, 5 \alpha \mathrm{OH}, 13 \alpha \mathrm{OH}$, DBAT, $10 \beta \mathrm{OH}$ and PAM, TS. The promoter of BAPT, GGPPS has fewer cis-elements, while the promoter of $14 \beta \mathrm{OH}$ has no corresponding cis-elements.

The activity of the three transcription factors also showed transcriptional activation activity against the promoters of DBTNBT, $2 \alpha \mathrm{OH}, 7 \beta \mathrm{OH}, 5 \alpha \mathrm{OH}, 13 \alpha \mathrm{OH}$, DBAT, $10 \beta \mathrm{OH}, \mathrm{PAM}$, GGPPS and TS, which contain cis-elements. However, there was no strong transcriptional activation activity for BAPT and $14 \beta \mathrm{OH}$. It is shown that the G-box cis-element is important for the activity of the above three transcription factors. However, the promoters of TAT and TBT, which contain a large number of cis-like 
elements similar to G-box, suggest that MYC transcription factors should bind to the transcription factors of the two genes. However, in the Arabidopsis protoplasts, they are not strongly activated by MYC transcription factors; in particular, MYC3 and MYC4 have a weak regulation effect on them. Whether the excessive cis-elements in the promoters of TAT and TBT lead to inhibition requires further analysis.

JAM2 has a significant inhibitory effect on all paclitaxel synthesis pathway genes, and its expression is induced by jasmonic acid signalling. It is likely to be a feedback inhibition signal of the jasmonic acid signalling pathway in Taxus. The inhibitory effect of JAM1 is weaker than JAM2. JAM2 can be combined with MED25. JAM1 and MYC4 almost have the same sequence. Besides the above functions, whether they are different from model plants, these two transcription factors have other unknown functions and need further research.

In summary, the jasmonic acid signalling pathway in Taxus is summarized in Figure 9. JAR1 catalyzes the binding of JA and isoleucine to form a biologically active jasmonoyl isoleucine (JA-Ile). It can mediate the Coronatine Insensitive 1-E3 ubiquitin ligase complex (SCFCOI1) binding to the jasmonic acid-inducing protein (JAZ) containing the ZIM domain. SCFCOI1 then labels ubiquitin on the JAZ protein. Furthermore, it promotes the degradation of JAZ protein bound to MYC by protease, stops the inhibion of MYC's activity caused by JAZ's binding. Eventually, activate the activity of transcription factors such as MYC2, cooperate with different transcription factors, recruit transcriptional complexes including Mediator25 (MED25), and activate genes expression in the downstream pathway. MYC transcription factors play a central role in the T. media signaling pathway, and include three MYC factors. The three MYC factors have an upregulation effect on the regulation of genes in each step of the paclitaxel synthesis pathway. In addition to MYC3 and MYC4 having an inhibitory effect of BAPT and $14 \beta \mathrm{OH}$, JAM1 and JAM2 are MYC transcription factors in T. media. They inhibit every step's gene expression in the downstream pathway.

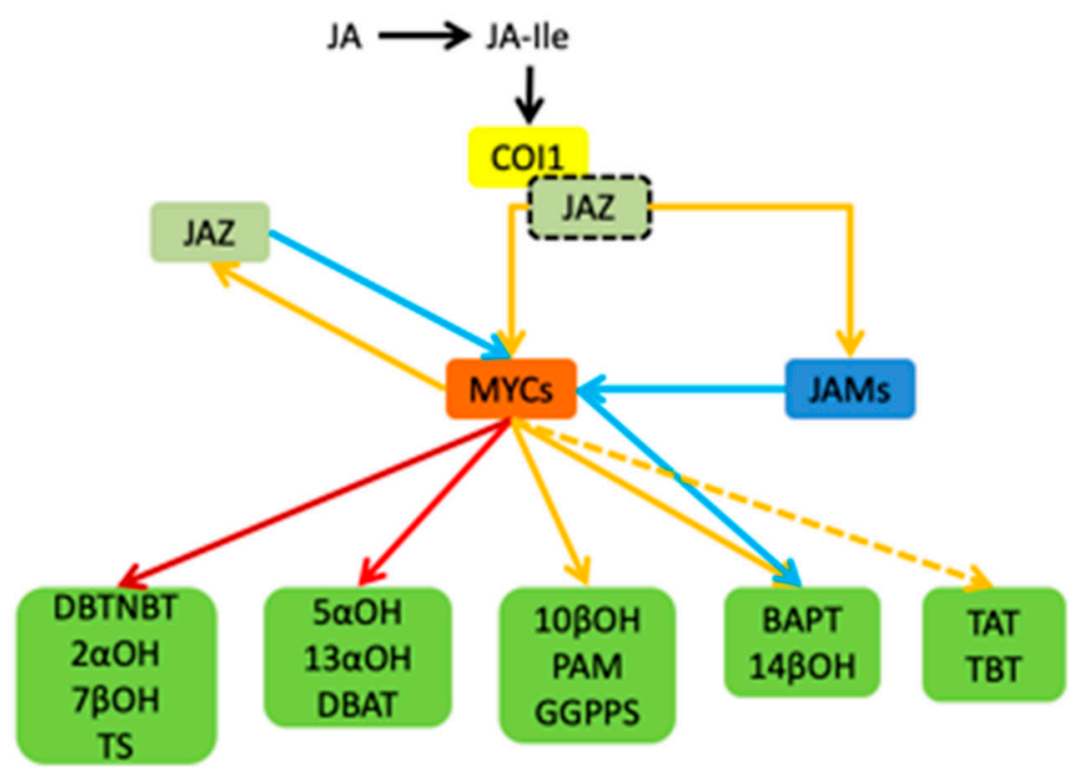

Figure 9. Schematic diagram of the jasmonic acid signalling pathway of T. media and its regulation of the paclitaxel biosynthesis pathway gene by the MYC transcription factor. The yellow solid line indicates that the upregulation or regulation is activated, the red solid line indicates a large upregulation, and the dark red solid line indicates a very large upregulation. The yellow dotted line indicates that there may be regulation, and the blue solid line indicates inhibition.

To confirm the function of the above jasmonic acid signalling pathway-related genes, the construction of related variants is the most important evidence, and the construction of the transgenic method of Taxus cell line is a worldwide problem, which is also related to this study. The study will 
validate the function of related genes and realize the construction of high-yield Taxus cell lines in the future.

\section{Materials and Methods}

\subsection{Cell Culture, Jasmonate Treatment, and RNA Isolation}

The T. media cell line Zike used in this study was induced from sapling leaf purchased in Beijing. The cells were subcultured using modified SSS medium. For jasmonate treatment, $25 \mu \mathrm{L} 100 \mathrm{mM}$ methyl jasmonate (MeJA) dissolved in ethanol was added to $2 \mathrm{~g}$ cells that were suspension cultured in $50 \mathrm{~mL}$ of medium 14 days after subculture. Samples were collected after 0, 0.5, 3, and $24 \mathrm{~h} \mathrm{MeJA}$ treatment using a Büchner funnel. Three biologically independent experiments were performed and a total of 12 samples were obtained. Total RNA was isolated from each of the samples as described before [25]. Generally, the cells were frozen in liquid nitrogen and ground to powder. Every $2 \mathrm{~g}$ of cell powder was lysed in $10 \mathrm{~mL}$ GTC buffer $(62.5 \mathrm{mM}$ Tris- $\mathrm{HCl}, 12.5 \mathrm{mM}$ EDTA, and $5 \mathrm{M}$ guanidinium isothiocyanate, $\mathrm{pH}$ 8.0), then incubated on ice for $15 \mathrm{~min}$ and centrifuged at $15,000 \times \mathrm{g}$ for $15 \mathrm{~min}$. The supernatant was added to an equal volume of isopropanol to precipitate the total RNA. The total RNA was purified using an RNeasy Mini kit (Qiagen, City, Germany) according to the manufacturer's instructions.

\subsection{RACE PCR}

The full length sequence of the $5^{\prime}$ and $3^{\prime}$ ends of the gene was obtained using the GeneRacer Kit with SuperScript III RT kit based on Invitrogen's RACE PCR technology (CA, USA) using high quality RNA extraction. First, the extracted RNA is dephosphorylated, and then the enzyme is used to remove the $5^{\prime}$ end cap of the RNA, and then the known RNA linker sequence is ligated. Using this as a template, the first strand of the cDNA is synthesized by the oligo(T) used as a locking primer to bind the the $3^{\prime}$ end ploy(A) tail of mRNA. Design specific primers for nested PCR at the $5^{\prime}$ and $3^{\prime}$ ends, respectively, clone the obtained DNA fragments, analyse the sequence of the coding region after sequencing, and after splicing, both stop codons and reach the longest coding region to determine the full length sequence at both ends.

\subsection{Yeast Two-Hybrid}

Based on Clontech's MATCHMAKER LexA Two-Hybrid System, JAZ and other related genes are ligated into a pLexA vector containing a LexA promoter binding domain (BD), and another gene for detecting interaction is linked to transcriptional activation domain(AD) of the pB42AD vector. The above two plasmids were then transferred to the lacZ-expressing active EGY48 yeast strain containing LexA control using the Frozen-EZ Yeast Transformation Kit of Zymo Research. After overnight culture in SD/-His/-Trp/-Ura liquid medium, transfer 5-20 $\mu \mathrm{L}$ of yeast culture solution to SD/Gal/Raf/-His/-Trp/-Ura/ for activity detection. In X-gal + BU salts solid medium, the appearance of blue spots was observed after four days of culture to evaluate the interaction between lac $Z$ activity and protein.

\subsection{Bimolecular Fluorescence Complementation}

The sequences of the two genes detecting the interaction were ligated into p35SnYFP and p35ScYFP, respectively, and the fusion protein was formed with the $\mathrm{C}$-terminus and the $\mathrm{N}$-terminus of the yellow fluorescent protein YFP, respectively. The assembled plasmids were then transferred to GV3101 Agrobaterium tumefaciens by electroporation. After induction with acetosyringone for two days, the two plasmids were simultaneously transferred into the tobacco leaves of the 4-week culture by injection, and then the fluorescence signal of the injection site on the leaves was observed using a laser confocal microscope. 


\subsection{Genome Walker PCR Separation Promoter Sequence}

To obtain the promoter of the paclitaxel biosynthetic pathway gene, the genome of T. media was extracted using the Plant Genomic DNA Purification Kit (Tiangen, Beijing, China). Based on Clontech's Universal Genome Walker Kit (Clontech, Takara, Japan), genomic DNA was digested with four different blunt-end restriction enzymes DraI, EcoRV, PvuII, and StuI, followed by ligation to the linker. Primers were designed for two rounds of nested PCR by known coding region sequences and linker sequences, and the obtained DNA bands were cloned, sequenced, spliced and analysed to obtain the promoter sequences of the corresponding genes.

\subsection{Detection of Transcription Factor Activity in Protoplasts}

To analyse the transcriptional activity, the transcription factor was transferred to a plasmid 35SGAL4DB containing a GAL4DB domain in a plant cell to form a plasmid expressing a fusion of the GAL4DB domain and a transcription factor. Based on the mature Arabidopsis protoplast transformation method [26], the Arabidopsis protoplasts were transferred by a PEG transformation method, and a plasmid containing a GAL4 promoter-controlled transcription factor was simultaneously transformed. The plasmid also contained the reporter gene for the LUC fluorescent protein in the plasmid and the plasmid for the continuous expression of the REN fluorescent protein internal reference. The fluorescence intensity changes of the two fluorescent proteins were detected using the Promega Dual Luciferase Reporter Assay system kit (Cat. E1910) (Madison, WI, USA), and the activity of the transcription factors in the plant cells was analysed by comparing the changes in the fluorescence intensity ratio.

\subsection{Detection of Transcription Factor Regulation in Protoplasts}

The promoter of the paclitaxel biosynthetic pathway gene was inserted into the coding region of the LUC fluorescent protein in the 0800 plasmid to construct a plasmid, which contained the reporter gene with promoter-controlled LUC fluorescent protein and the continuous expression of REN fluorescent protein as internal reference. At the same time, the transcription factor to be detected is inserted into another plasmid 62sk, and the transcription factor can be continuously expressed in the plant by $35 \mathrm{~S}$ regulation. The two plasmids were co-transformed into protoplasts by PEG transformation. The fluorescence intensity of the two fluorescent proteins was simultaneously detected using the Promega Dual Luciferase Reporter Assay system (Cat. E1910) kit, and the regulation of the transcription factor on the paclitaxel biosynthesis pathway promoter was analyzed.

\section{Conclusions}

In this study, based on the jasmonic acid signalling pathway of the Arabidopsis model, the jasmonic acid signalling pathway of T. media was isolated and identified. The interaction between related proteins was analysed, and the molecular mechanism of the jasmonic acid signalling pathway in Taxus was constructed. Further analysis of the activation or inhibition of the promoter of paclitaxel biosynthesis pathway gene by transcription factors such as MYC2 confirmed the regulation of MYC2 and other transcription factors on the biosynthesis pathway of paclitaxel. Based on this design, the transcriptional activity of MYC2 and MYC3 was not affected by the JAZ inhibition function, which laid a foundation for the construction of a high-yield paclitaxel variant Taxus cell line.

Taxus has a corresponding jasmonic acid signalling pathway, and it directly regulates the expression of paclitaxel biosynthesis pathway genes through MYC transcription factors, and JAZ and non-transcriptionally active MYC transcription factors comprehensively regulate the expression of related genes. Based on the signalling pathway, a variant MYC transcription factor was constructed to activate transcription without inhibition by JAZ protein, and was the basis for constructing a taxol-producing Taxus cell line. 
Author Contributions: Conceptualization, J.C. and Z.G.; methodology, Y.C., J.C. and Z.G.; software, R.M.; validation, Y.C. and R.M.; validation, Y.C. and R.M.; formal analysis, R.M.; investigation, Y.C.; resources, Y.C.; data curation, Y.C. and R.M.; writing-original draft preparation, Y.C.; writing-review and editing, Y.C. and Z.G.; visualization, Y.C. and Z.G.; supervision, J.C. and Z.G.; project administration, J.C. and Z.G.; funding acquisition, Z.G.

Funding: This research was funded by China Postdoctoral Science Foundation.

Acknowledgments: The authors would like to thank Daojun Xie and Jiaojiao Wang from the Department of Biology, Tsinghua University, and Susheng Song from the School of Life Sciences of Capital Normal University, for their technical support.

Conflicts of Interest: The authors declare no conflict of interest. The founding sponsors had no role in the design of the study; in the collection, analyses, or interpretation of data; in the writing of the manuscript, and in the decision to publish the results.

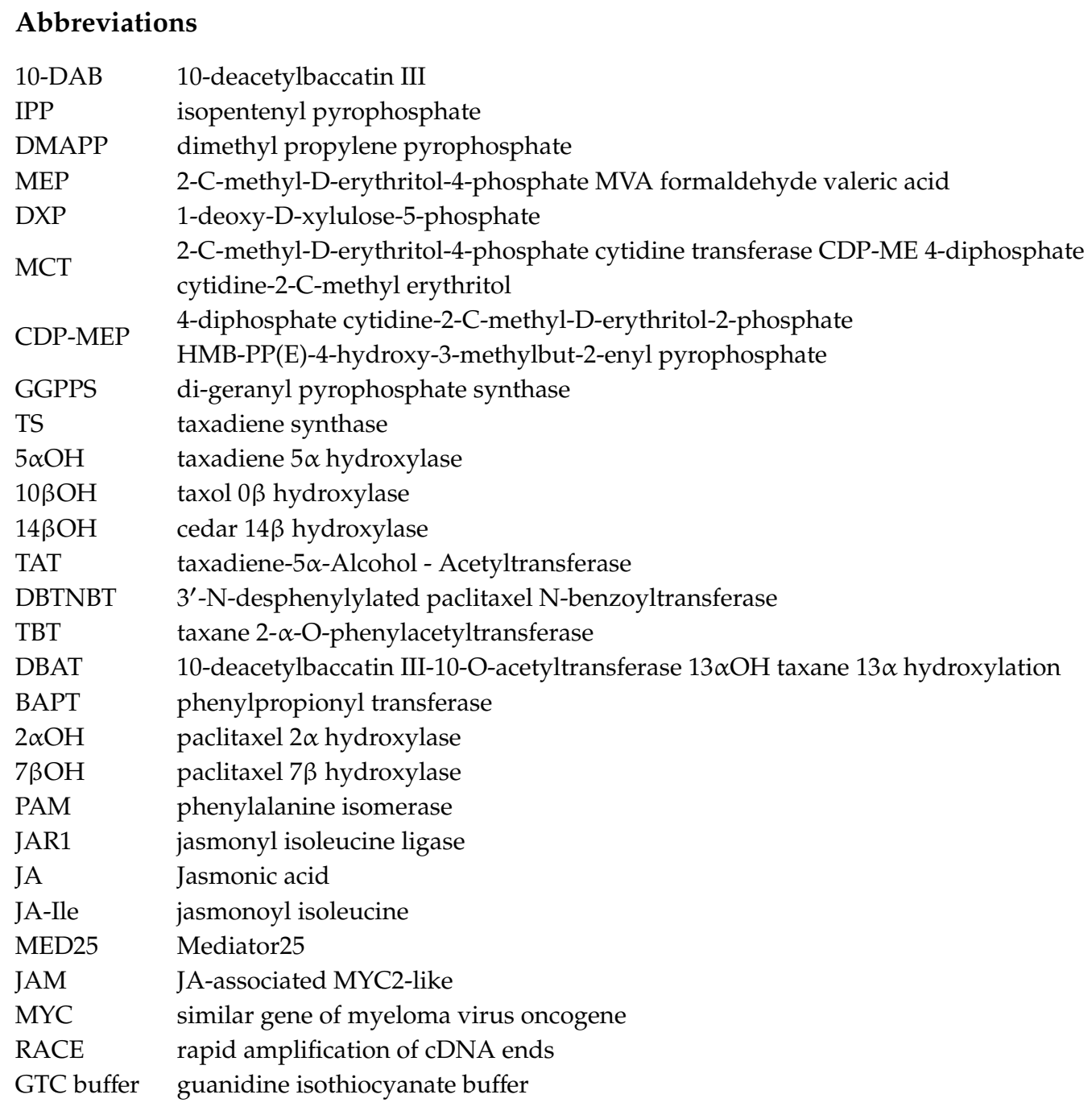




\title{
Appendix A. Gene Sequence
}

\author{
$J A Z$ sequence
}

$>$ JAZ1.1

ATGTATCACGCAGAAGCAGAGGCGGAGCGTGAGCCTCGGCTGTTAACAATTGATTTTATGGGAATCGGATCTGACAAGCTCG AAACTAGTAACAGTTGTTGCCACCAACAACTTACAACCAGGCGATCAATGCAGTGGTACCTGAGAAAGATAAAACCCCATAT GCTGCAGCAAGTGTTTTTCCCCAATCCTGAGGTTGAGATTAGTCAGCCTATAATCAAGACCTTGGAATTGTTTCCGCTACACAC TCCACGGGTTGGATACAATTGTAAAGAGGGCGACATTGTGGACCGCCAATCGCCTTCTATCACTGCTCCGATTAATTTTTTTGA AGCTGCCCCTTTCTACAATGACGCACTGAACGTTTCTTCTGAAAAAGTGGGTAATGGAAACGGAAATGATCTGCAACATATTT ATCCGGCATCTGAGAAAGGCGTATCTTCCACCAACAGAGGGGCAGGGGCCGGATTCACAGCCACGCAGAGTGTTGAGGCAC CACGATCCCAACTTCCGATAGCGAAGAAATTATCTATTCAACGTTTTCTCCAAAAGCGAAAGGACAGACTTAACAAATTACTT CCCTACACGACTTATTCAAGGGAAGGCTGCATAAGTAATTGTTTATTTCTAAAATTGGAGAGCGGCGCGGAAGAAAGGATTTG GAGACACATCGATTAG

$>$ JAZ1.2

>JAZ1.2 AAACTAGTAACAGTTGTTGCCACCAACAACTTACAACCAGGCGATCAATGCAGTGGTACCTGAGAAAGATAAAACCCCATAT GCTGCAGCAAGTGTTTTTCCCCAATCCTGAGGTTGAGATTAGTCAGCCTATAATCAAGACCTTGGAATTGTTTCCGCTACACAC TCCACGGGTTGGATACAATTGTAAAGAGGGCGACATTGTGGACCGCCAATCGCCTTCTATCACTGCTCCGATTAATTTTTTTGA AGCTGCCCCTTTCTACAATGACGCACTGAACGTTTCTTCTGAAAAAGTGGGTAATGGAAACGGAAATGATCTGCAACATATTT ATCCGGCATCTGAGAAAGGCGTATCTTCCACCAACAGAGGGGCAGGGGCCGGATTCACAGCCACGCAGAGTGTTGAGGCAG CACGATCCCAACTTCCGATAGCGAATGCCAAGAGGATAAGATGA

$>$ JAZ2.1

ATGGGAATCGGATCTGACAAGCTCGAAACTAGTAACAGTAGCAGTAGCAGTTGTTGCCACCAACAACTTACAACCAGGCGAT CATTGCAGTCGTGCTTGAGAAAGATAAAACCCCATATGCTGCAGCAACTGCTTTTCCCCAATCCTGAGGTTGAGATTTCTGAG CCTATAATCAAGACCTTGGAATTGTTTCCGCTACACACTTCCAATTGTAAAGAGGGCGACATTGTGGACCGCCAATTGCATTC CATCACAGCTCGGATTAAATTTTCTGAATTAGCTGCCCCTTTCTACAATGACGCACTGAACGTTTCTTCTGAAAAAGAGGGTA ATGGAAACGGAAATGGTCTGCAACAGATTTATGCGGCTTCTGAGAAGGGCGTCTCTTCCACCAGCAGAGGGTCAGGGGCAG GGGCAGGGGAAAGGGCAGGATTCACGGCCACGCAGTGTGTTGAGACACCACGTTCCCAACTTCCAATAGCGAAGAAGTTAT CTCTTCAACGTTTTCTGCAGAAGCGGAAGGACAGAGTTAACAAATTACTTCCCTACACGACGTAA

$>$ JAZ2.2

ATGTACCAGGCAGAGCCAGAACCAGAGCTAGAGCGTGAGCCTCGGCTGTTAACAATTGATTTTATGGGAATCGGATCTGACA AGCTCGAAACTAGTACCACTACTGGTTTTCAACTTACAGCCAGGCGATCATTGCAGTCGTGCTTGAGAAAGATAAAACCCCAT ATGCTGCAGCAACTGCTTTTCCCCAATCCTGAGGTTGAGATTTCTGAGCCTATAATCAAGACCTTGGAATTGTTTCCGCTACAC ACTTCCAATTGTAAAGAGGGCGACATTGTGGACCGCCAATTGCATTCCATCACAGCTCGGATTAAATTTTCTGAATTAGCTGC CCCTTTCTACAATGACGCACTGAACGTTTCTTCTGAAAAAGAGGGTAATGGAAACGGAAATGGTCTGCAACAGATTTATGCG GCTTCTGAGAAGGGCGTCTCTTCCACCAGCAGAGGGTCAGGGGCAGGGGCAGGGGAAAGGGCAGGATTCACGGCCACGCA GTGTGTTGAGACACCACGTTCCCAACTTCCAATAGCGAAGAAGTTATCTCTTCAACGTTTTCTGCAGAAGCGGAAGGACAGA GTTAACAAATTACTTCCCTACACGACGTAA

GTAZ2.3

ATGAATATTGATGTGGCCAGGGGTTTATTGTACACAGCATTGCAGTCGTGCTTGAGAAAGATAAAACCCCATATGCTGCAGCA ACTGCTTTTCCCCAATCCTGAGGTTGAGATTTGTCAGCCTATAATCAAGACCTTGGAATTGTTTCCGCTACACACTTCCAATTGT AAAGAGGGCGACATTGTGGACCGCCAATTGCATTCCATCACAGCTCGGATTAAATTTTCTGAATTAGCTGCCCCTTTCTACAAT GACGCACTGAACGTTTCTTCTGAAAAAGAGGGTAATGGAAACGGAAATGGTCTGCAACAGATTTATGCGGCTTCTGAGAAG GGCGTCTCTTCCACCAGCAGAGGGTCAGGGGCAGGGGCAGGGGAAAGGGCAGGATTCACGGCCACGCAGTGTGTTGAGAC ACCACGTTCCCAACTTCCAATAGCGAAGAAGTTATCTCTTCAACGTTTTCTGCAGAAGCGGAAGGACAGAGTTAACAAATTA CTTCCCTACACGACGTAA

$>$ JAZ3

ATGGAAAGGGAGTCGGTTGTTGCGAGGGACTTTTTGGGTCTGAATCATGCCAGAAATAATCTCAGTGGGGACTCACAAACTC AGACAGAATCCCAAAGAGACGGGCCTATTAAGGGCTATTTCAAAGATAACGTATCCCTGGCAAATATGTCTTTGCAGTGGCCT TATTCAAATAAAGCAGCTGCTCTGCAGCAGTTCATTTCTTTTAAAAATCTGCAGGAGGGATCGAAGAAGAGTTCATTTGACCA GCTTTCAAATTCTGGGGTTCATCCTATATCCACTGCAGATGTTTTTGATGTAAACAACAGAAATACAACTGGTTCTCAGAAAGC CTTCAATCTTGATCCTGCTGCGAGGAATGCCACGGAGTCTGGAAACTATCATTTCGGGCAAAATGGGTCAGTGAGCCGACCTT TCAGTGTCTCTCGTATCATGTATTCAGCTAAGACAGTTGATGATTGGGCTTCTCAAGGCTATAACAATCAAGAGGGTCGAGTGT TTCCCAGTTCAA ATTATTCTCACCCGGTTTCTAATTCAAATTCAAGCATTCCTGCATACTTCAATGGACATCAAGCAACGGCTA CATCTTCACTCACGACACAGCAATTTCAAGGAATTCCCTTAACAGGACAACATTCTTTCTTGCCTATTCTTGGAGCCTCTGCCA GACACTCAATACTAGGGTGTGGTCCTAGTGTTCCTGAAAAGCCAGCAGGTGCACAACTGACAATATTCTATGCTGGGTCAGTA AATGTGTATGACGATATTCCTGCAGACAAGGCTCAAGCAATTATGTTTATGGCTGGCAATGGAAATCCCTCTTCTGGAAAGAT AGCGACTCCTCCGCCGCAAAGCTCAACAATTTCACTGTCAGCTATTGGGCATGTTAAACAAGCTACATCTGTGGGGCCAAGC CCATTAAATGCATTGCCTGCAACAAATCCACAAGTCGATTCCCAGAAGTCAGAGACAACTGTCCAAAGTCAAACTAGCCAAT CTACTGCAATATTTCCTAGCACTGGAGATCCAGATGCAAACCAAAGTAATGCTACTTTGAGTAATCCACAGGAAACTCCCTTG AATGCTCCAGGGTCAACGCCAACATCTATTATGTCTCGAGCGGTACCACAAGCTCGTAAAGCATCACTTGCTCGATTTCTTGA AAAGCGCAAAGAAAGGGTCATTACAAAAGCGCCTTATCCAACAAAGAAGTCACCAGAGGATTCCTCTCTTTCAGACAAATC TCTTAGTCCTAAGTGTTCATCTACACCACCCATGGATGGATGCAAGTCACAACATGAGTCTATGACAAATTCAGAAGAAAAG TCTTAGTCCTAAGTGTTCATCTACA

$>$ GGCTCA

ATGGACCGAGAGAAAATTGTCGAGAGGGATTTTATGGGGTTAATTAACAGCGATGAATTTGCAGACAAAAAAGAGGACGAC TCTGTAACTAAAGATTCATGCACTGATGCTCCTGTGGACGATGGGAGCCACAACTCTCTTCCAAATAATGATGTACCCTGTCA ACATTTTGGAACGTCTTCAATGGCATATCCGGCTGGATCAACGGAGGGATTGAGCTCTTCAGTCCATCCTGTTGTTGAATATGG AGTCTTTCCGAGCTCAAATATTTCACTTCCAATGCAAAATTATGGCCAGCATTTAAGTGCACTTGCTATTTTGAAAGGGCAGCA AGCAGTATCTAGTGCCTGTGGTGCATCTTCTATGGCTAATCCTTTCCCCATCCAGCATTCTTTTTTTCCAGATTGTGCTGGTGTAT ATCCTACCACAAGAGCAACAAGCAAGTCTACTACCATTCCAGGAAATCCTGCACAGCTCACAATTTTCTATGCCGGGACAGT GAATGTGTATGATGATGTCCCTGCAGACAAGGCTCAAGGTCTCATGCTTTTGGCCGCAAGTGCAAGGCTTTCAAAAACAATC AATCTGCCTCCACGAAGTTCATTAATGTCAATGTCTGTGGCAACTGGTTGTCATACTCCATCAATTGCATCACCATTTACCTCA AGTTTGCCAAATCAAGCATGTAATACGACTCCACAAGCTCCTTTACAAAAGCCACAGGAAAACAATCAGCCTAATTCATCAA CAGCAGTATCATTGAATGCTGGAGACTCTCAGAAAAATCAATGCATTGTGACTTCGATTGGCCAACAGGAAGCTTCCATCTCA AGTGCCATGGTGATAACCCCGGTTCCTGTTGTACCAAGAGCAGTACCACAGGCACGCAAAGCATCCCTAGCTCGCTTTCTTGA AAAACGAAAAGAAAGGATCAGTATAAAAGCTCCATATCCTACTAAAAAGTCACCTGATGCTTCACCTCAAAGAGAGCAATC TCCTAGTTCTAAACATGCTTCTTCTCCTCTAGATGAATGCCTAACAAAAAACCATCAGGTGGCCAGTACTGGACTGGAGGAGA AGATTTCGTTTGATTCAGAAAGCATAGGATCTTTTCAAAAGGATCATTCGTGTTTACAAAAAGCTCCAAAGATTGAGGGAGAG AGATTTCGTTTGATTCAGAAAGC GACT
$>$ JAZ5

ATGGCCCGAGGGGAGCTGGTGACCATAGACTTCATGGGACTCGACAAGGGAAAGGATGCGGTTAAGGAAAAATGCGTTCAT GATTTGTCGTGGATAAAGCCGAAGATGATGCAGCAGGTTCTTTCCTTGAACAAGCAAATCGATACCGTGCAGGCATTGAAAG GATTGTCTTCTCATTCTGTTCCAAATTCCAGATCCGTTTCTCCAAATTTATCAGGAAAGCAATCCGGTACTACTGGTACTGCGC AGCTGACCATTTTTTACAACGGGGAAATAAACGTCTATGATGTTTCTGCAGAGAAGGCTAAATCTATCTTCATGCTGGCCAGC GGTGAAAATAATTCCAATCAATTATCACAGACTGAAGAGCCGCAGAATTTTAGTGCCCTAGCTGAGCAGGGACAGATTGCAA AATTACACGGCGATCTTCCGATCGCAAGGAAACAATCTCTGCAACGTTTTCTGCAGAAGCGGAAGGACAGGATGATCATGGC GGCTCCTTACTCTCAAAAGGAACAAGGCAAGTGCGACATCATTGTAAAGCAATAG 
$>$ JAZ6

ATGGAAGCGTTGAGCATTTACAGAGGAGGGAAACTGCGAGTTCCGGAGCCTAGAGCCGGCATGCAAGGTCTGTCGTGGATA AAACCGGAAATGATGCAGCAAGTGCTTTCCTCTATTGTCCGTCCTCAAGAACAAATCAAGGTACACAGTCAAGCGGAATTAA TGGATTTATTTCGTTCAGAGCGCGAGCACGACGCAACCCTCCCCCTATCAGATCGCAGATCTGTGTCTGAAACCCAGGATAAG CAATCCACCACTCAGCTCACCATTTTCTACAATGGCGCCGTGAATGTGTATAATGTTTCCGCTGAGAAGGGCGAAGCAATTCT GGAACTTGCTAGCAACAATAGCTTAGGGAAAACGGCAAGCCATATAGCTTCTGGCGAAATCAAAGAGGAAATACTGAAACC CTCTGCCAGGCTTCCAATGGCGAGGAAATTATCTCTGCAGCGCTTCCTCCAGAAGCGCAAAGACAGATTGAGCACAGCTGCA CCATACAATGTCACGTCCACGTCAAATACACCTTTGAATGTTTCAACATCAACTACAGCAAAAGATAAGAATTCAGAGGAGC ATATTAGGCTTTCTTTGTCTTTTCCGTCACAAAGATCTTGTTAA

$>$ JAZ7

ATGTTGCAGGAGGAGAGCGATGGAGAAATGAGCGGCGGAAGCGAAAGTTCATCGCTTCTTAATTTGGATCTCTCACTTTCGC CGTTCAAAACAGGCGGCGGCCATGATCAGAAGCAGAAGCAGAAGCAGAAAGAGGTTGCCCAACTCACCATTTTCTACAGG GGCACAGTCAATGTTTTCAACGTTTCGCCCCAAATGGTAAAAACAATAATGATGGTAGCAAACACGGAAAGTCCTCCGCCTA CGCCTTCGTTACAGATGTCTTCTTCTTTTTCAGCAGGCCGCACCCTCACCTGCCCTATTCTCACGCCAAAAATGGTAAATCTTC CGATCACTCGGAAAAAATCACTGCAGCGTTTTCTGGAGAAGAGGAGCGACAGGGTTCACGGGAGGGCTCCCTATGCATCTCT TCCTCACGCACACACTTAA

$>$ JAZ8

ATGGATTTTATGGGATTGAGTGGCAATACCAGTAGAACCCAAATGGAGAGACAGGGGAATAGGCAACACCCAGATGATGAA AGTGATAAAGAACTGAGTCTGAGTCTTTCCCATGAGGCTCACAAGACCGGTCCGGGGAGTGGGCTGCAGCTTTCTTTATCTG GCAAGAGTAAGGGTTGCAGTGTTGAAGGTGGTGATCAAGTAGCTCTGAATCTGGGTAGTAGCAGTCCATTAAGGGGGACTGT TGTCAAGGTTGGGAATGTAACCGGATCTGCTAGCTTTTCTGCATACAATCCTGAGAAGCAGAGGTCTTTGTTAAGGTTTCATGC TCTTCCTAAGGGAAAAATTCTCTCTGGAATTGAGAACCGCCACCTAAATGACAAGCCAAAAGCTGTAACTGTGGTAGCAACA GGCAAGCTGCCTGATAACCTTGCAGAATCGGGTTTTCAACACCAAAAACCTTATTCTAAAGATAAGGCACCTGTGCCAAACT TGAGTATGCAGTGGCAACAGCTCATGTCTATAAAGAATGCACAGGAAGATAGGCCTGCCAAGAGGAAGTTCGACTGGCTGG CAAAGCCTGTTCCAACTGCAGATGCTTTCAAACCAAACAAGAGAAGTACAGTGGCTTCACAGAAATCCTACAATTTCAGTCC CACATCAAATGATACTCCAGAGTCTAGAAACTTTTACTCTGTAGCAAATGGTAATCTAAATCAACATTTTGGAGCATTTCCTGC AACATTTTCACCTCGAACATTTGATGATTGGAGCTCTTCAGATTACTGTTATGATCAAGCTCAAAGTTTTCTTACAGTAAAATCT TCGCACTTGCCTTCAAATGTCAATGAGTGTTTAGTCTCTCCTTTGAAGGAACAGCCAGCAGCTAATTCGTCTCAACCACAC $>$ JAZ9.1

ATGGAAGAACATGCACAGTGGAGCGGTGCACCAGCCACATCCTACGATGCAGATGATTCTTCTGATGGTGAAGCCAGCGAA AATGAAGCACGTTTTTGCATTAAGGAATTCTTAACCCTAAAAGAGAAAGCTTCTGTGGAAATGGAGAAGAATATTGAATCAG ACAAAGATAGAGGTAAGAGCGTAATAGAAAACCAGGCGCCTAATTTGGATCTTTCCCTCTTGCCTGCTTGGAAGGCGGTGGA GCCACAGAAAAGCCCACTTGGTGTCTCCCCTTCTATAAATGCTAATGCTTTCAGGTCTACTTTCACACAGAGCAAGAACCAAC CGGGACATAACCAACTCACCATTTTCTATAGAGGAACAATGAATGTTTACAATGTGACACCTGATTTGGCAAAAGCTATAATG TTGGCAGCAAACAATAATAACAGTGCATTGTGTCCCACTCCCTCGACATGCCACACCAGTTACATCCAACCTGCTCTCACGCC CAAAACTGATTCAAAGATTCCAATTGCTCGACGAAATTCTCTAAAGCGTTTTATGGAAAAAAGGAAGGACAGGGTTCTTTCT AGGCCTCCATATTCCACTTGTCACCATCATTAA

$>$ JAZ9.2

ATGGAAGAACATGCACAGTGGAGCGGTGCACCAGCCACATCCTACGATGCAGATGATTCTTCTGATGGTGAAGCCAGCGAA AATGAAGCACGTTTTTGCATTAAGGAATTCTTAACCCTAAAAGAGAAAGCTTCTGTGGAAATGGAGAAGAATATTGAATCAG ACAAAGATAGAGGTAAGAGCGTAATAGAAAACCAGGCGCCTAATTTGGATCTTTCCCTCTTGCCTGCTTGGAAGGCGGTGGA GCCACAGAAAAGCCCACTTGGTGTCTCCCCTTCTATAAATGCTAATGCTTTCAGGTCTACTTTCACACAGAGCAAGAACCAAC CGGGACATAACCAACTCACCATTTTCTATAGAGGAACAATGAATGTTTACAATGTGACACCTGATTTGGTAAACTATGATTGA $>$ JAZ10

ATGACCCAGGAAAGATCTGATATTATGCAAATGTTTGTACAGCAGCAAGCCATGGATCAGGATTCCACCCAAAAATCCGGTTT TGGTTCACCTGTGGCTGCTACAGCAGACGTCCAACACAGAAACCTCCATCTGCACCCACTGTTCAGGTCGGCTGGGTGTGCC GTTGCAGAGAAGCAATCCGGGAGTGCCCAGTTGACGATTTTTTATGGGGGAATGGTGAACGTTTATGATGATATTTCTGCAGA TAAGGCACAGGCCCTAATGTTAATTGCCAGTACTGGCAAACCCATGCAAAAGAACAGCAGTTATGCATCAGAGCCTCAACCC ACAAAATCAGTCAAACTGTCCACCGTTAACTCAACTGAGTTGGAATTGACCGTAGATCTTCCGATTGCGAGGAAGAATTCTCT GCAACGCTTTCTGGAAAAGCGAAATCACAGGCTGAATGCAAAGGCTCCCTATACCATTTCACAAGAAAATCCTAAACCAAGT CCATGTCTCTGA

$>$ JAZ11.1

ATGACCCACATGAACCAGGAAAAATCTGGTATTATGCAAATGTTTGGACAGCAGCAAGCTATGGATCAGGATTCCAGCCAAA AATCTGGTTTTGGTTCAGTACCCCTTCCTGTGGCTGCAGGATTCCAACACAGAAAACCCCATCTGCAAGGCCTGTTCCCAATG TTCAGGCCAGCCGGAAAAGAAGCCCGGGGTGCCCAGTTGACGATTTTCTATGGGGGAATGGTGAACGTTTATGATGATATTTC TGCAGATAAGGCACAGGCCCTAATGTTAATTGCCAGTACTGACAAACCCAAGCAAAAGAACAGCAGTTATGCATCAGAACC TCAACCCACAAAATCAGTCAAACTGTCCGCCGTTAACTCAACTGAGTTGGAGTTGACCGTAGATCTTCCGATCGCGAGGAAG AATTCTCTGCAACGCTTCCTCGAGAAGCGAAATCACAGGGTGAATGCAAAGGCTCCATATACCATTACACAAGAAAAACCTA AACCAAGTCACATCTCTGGTTAA

$>$ JAZ11.2

ATGACCCACATGAACCAGGAAAAATCTGGTATTATGCAAATGTTTGGACAGCAGCAAGCTATGGATCAGGATTCCAGCCAAA AATCTGGTTTTGGTTCAGTACCCCTTCCTGTGGCTGCAGGATTCCAACACAGAAAACCCCATCTGCAAGGCCTGTTCCCAATG TTCAGGCCAGCCGGAAAAGAAGCCCGGGGTGCCCAGTTGACGATTTTCTATGGGGGAATGGTGAACGTTTATGATGATATTTC TGCAGATAAGGCACAGGCCCTAATGTTAATTGCCAGTACTGGCAAACCCATGCAAAAGAACAGCAGTTATGCATCAGAGCCT CAACCCACAAAATCAGATCTTCCGATTGCGAGGAAGAATTCTCTGCAACGCTTCCTCGAGAAGCGAAATCACAGGGTGAAT GCAAAGGCTCCATATACCATTACACAAGAAAAACGTAAACCAAGTCACATCTCTGGTTAA $>$ JAZ12.1

ATGGATTCCTCACTAATTCATAAGGGAAAGCGAAAATTAATATATTCGGACGAAGGAAACAGCGAGGCAGCCGAAGCCAAC TCTGCGGAGGAGGGCGGACCCAGCGAAAACAAGCGTTGTTCTATCACATTCACGCAACTCGAGGAAATGGAATCAGAAGAC GAAATGAGCCGCGATTCCGTTTCAACTCCGATTATGAGTTGCAGGAGAGGAATGCTTAATCTGGATCTTTCCCTCTTGCCATCC ACACATTCGTTTCAGGCCTTTTCTCCTCTAAATTGGGGAGGACCTTCTCCCTCTTTCAGGCAAGTGAGATCTTTTAACAAGCAG CAGGAGCAGGAGCACGGGCAACTCACAATTTTCTACGGGGGAGCGGTGAATGTTTACAATGTTTCGGCCGACAAGGCAAAG ACTATAATGATGATGGCCAGCAAAAGTGCTTCTACTCCAACGCCAACGCCGCTTAGCGATATGCAAATGTTTTACAAAAACAC GCCGTTGAAAGCAGGTAAAAGCAGAGGTGCCTAA

$>$ JAZ12.2

ATGGATTCCTCACTAATTCATAAGGGAAAGCGAAAATTAATATATTCGGACGAAGGAAACAGCGAGGCAGCCGAAGCCAAC TCTGCGGAGGAGGGCGGACCCAGCGAAAACAAGCGTTGTTCTATCACATTCACGCAACTCGAGGAAATGGAATCAGAAGAG GAAATGAGCCGCGATTCCGTTTCAACTCCGATTATGAGTTGCAGGAGAGGAATGCTTAATCTGGATCTTTCCCTCTTGCCATCC ACACATTCGTTTCAGGCCTTTTCTCCTCTAAATTGGGGAGGACCTTCTCCCTCTTTCAGGCAAGTGAGATCTTTTAACAAGCAG CAGGAGCAGGAGCACGGGCAACTCACAATTTTCTACGGGGGAGCGGTGAATGTTTACAATGTTTCGGCCGACAAGGCAAAG ACTATAATGATGATGGCCAGCAAAAGTGCTTCTACTCCAACGCCAACGCCGCTTAGCGATATGCAAATGTTTTACAAAAACAC GCCGTTGAAAGCAGCAAATCTTCCCATCAGTCGAAAGAATTCTCTGCAACGGTTTCTGGAGAAGAGAAACCACAGGCTGTAT GCGAGGGATCCCCATGCCCGTGTTGA 


\section{MYC sequence}

\section{$>$ MYC2}

ATGATGGGGAACGAGGGGACAGCGTCAATGCGTTTGTGGAACGAGGACAACAATGCCCTGATTGAGGCGTTCATGGGGCAC ATGGACTACTCTTTCCCCTGGAATGGCATTGACGCCAATCCCTCGCCTGCACCAGCACCAGCACCAGCACCAGCCCCTATTTT GACAGCCCCTCCGCCGCAATCGTCATTCAACCAAGACACACTGCAGCAGCGCTTGCAGGCTTTGGTGGAAGGCGCTTCAGC AAGCTGGACATACGCCATTTTCTGGCAGATTTCCTCCGACCCAGAGAACGCAATGGTCTTAGGCTGGGGCGACGGCTACTAC AAGGGTCCTCGAGATTTGACGGACGAGGAAAGTGCGTCAAAGAGGGTTTCTGCTTCTTCCTCCGCTTTTGAAGCGACGGCTT CGGATCAGGAGCTCCGCAAGAAAGTGCTTCGAGATCTCCACACGCTCATCAACCCCGACATTGAAATGACTGATATTTCATC GACAGTTGACGGAGAAGTAACGGACGAGGAGTGGTTTTATCTGGTGTCAATGATGCAGTCGTTTGTCAATGGATGCGGCGTG CCTGGGCAGGCGTTTTTTACTGCAACGCCCATTTGGATTACAGGCCCCGAGACACTGCAGGCTTATAACTGTGACAGGGCCCG TCAGGCCCAGCAATTTGGAATTCGGACTTTGGTATGCATTCCGTCGCCCAACGGAGTTGTGGAGTTGGGTTCCACGGATTTGA TTACACAGAACTGGAATTTGATGCAGCAAGCAAGGAATTCATTCACTTTTAACGACAACCCAAATCCTCTATGGGAAGAAGG GAACCCTAGCTATAACAACAACAATAGCGTCGACGCTGATCCGTCGCTTTGGTTGACGGAGCCGCCTCACGTTGAAGAGCAG CCGCAGGCAGTAGATGCGGAGAAGCCCAAACAACATACAGAAACCGAGGGCGTTAACGGGTTTTTTGAACTGGGGTTTTCG GACCTAGGGTTTGGGGTTGATCGTCAATTGACGGGAGCATTTGAAGACCGTCAATCGACTGGAATTTTACAACCAGGTACAC AGATGGTGTACCCGTCCTACAAATCAGAGGCCGTAGAGAGCACTCAGGCTTTGCAGAATGGAAATAGACAGCAAGTGGCTTT GGATGGGGATGGCGATGGGGATGGGGATGTAATCACTCTGAGCTTTGCCGAAGCTCGAAACGATGACCCTGCACAGAGCTTA GTGGCTTCAGCTACGGCTGCTGCTGTTTTGCCGATGAAGTCTAGTGTTGAATCAGAGCACTCTGACATTGAGGCCTCCATTAA GGAGCCGGAGTGCAGCCAGGCTACTTTTGTGGAGAGGAAGCCCAGAAAAAGAGGGAGAAAGCCTGCAAATGGCAGGGAG GAGCCACTGAATCATGTGGAGGCTGAGAGGCAGAGGAGGGAGAAGTTAAACCAGAGGTTCTATGCACTCAGGGCTGTGGTT CCCAATGTGTCCAAGATGGATAAGGCATCTTTGTTGGGCGATGCTATTGCTTATATTAATGAATTACGAAGCAAGGTGGTGGAT GCAGAGACTCATAAGAAGGAGCTGCAGGTCCAAGTTGAGGCATTGAAGAAGGAATTGGTGGTGGTTAGAGAATCTGGTGCT TCTGGGCCTAATTTTGGCTTGATCAAGGATCACTACCCCACAGCTGATTCATCTGATGTCAAAGGCCATGGCTTGAATAACAGT AAATGTCATGGTATAGAGCTCGAGGTTCGATTACTTGGCAGAGAGGCAATGATCAGAGTTCAGAGCCCAAAACAGAACCAC CCTGTTGCAAGGTTGATGGGGGCATTGAAAGAGCTCGATCTTGAGGTCCACCATGCCAGTGTATCAACGGTGAAGGAGCTGA TGATTCAAACAGTTATTGTGAAGATGACAGGAGGTATTGTATATAGTCAAGAACAACTAAATGCAGCACTAAGCAAGTCAGTT GCAGATTCTGGCCTTAGGTAA

\section{$>$ MYC3}

ATGGCTCTGGCGGGAATGAAGTTGTGGAGTGACGATGCCATGGTGGAGGCGTTCATGGCGTCCTCCTCTCCGCAGGTGGGAG TTGGGTGGAGCAATGGCGGCGCCACTGCGACTGTGTCTCCGTGTAACGACAATTCCTTGTTTAATCAGGAGACGCTACAGCA GAGGCTTCAGATGTTAATTGAGGGCGCCCAAGAGAGTTGGACATACGCCATATTCTGGCAGTGGTCGTACGACCCAAGCGGC GCCATCGTTTTCTGCTGGGCCGACGGCTACTTCAAAGGCGGCGGCGCCGCCGAGGAGGAGAAGCAAAGGAGCGCAGTTGA GCAGGAGGTGAGGAAAAAGGTGTTGCGGGAGCTGCACGCGCTCATTAACGGCGGCGGCACCGAAGCAGATCCCCCGGCTG AGGCGGAAGCGGAAGCGGATGAGGAGGTGACTGATGCGGAGTGGTTTTATTTGGTGTCGATGACGCACTCGTTTGGCGGCGG GCAGGGTGTGGCGGGGCAGGCGTATCTCAGCGGTTTGCCGGCTTGGCTGTCTGGGTCGCAGAAAATGGAGGGGTGTCATTGC GAGAGAGCTAAACAGGCGGCTCAGTTCGGCATTCAAACCATGGTATGTATTCCTACTCCCAATGGCGTTGTGGAATTGGGGTC TGTGGTTTTGCTTGCTCACAATTGGGAGCTGGTTCAACAGGCTAAGACCTCTTTTACTTTTTCGTCCTTTGGCTTGTGGGAAGA TCATGGAAATAATAACAACACTACATCTTCCGACAACAATACCAATCTCATCATCAACAACAACAACGACAACAACTTTTGT AACAATAACCAGTCTCTGTGGGTTCCGGGTTCCCCTTTTTTCGGTGGCCATGCCCAGGAATCTCCCCTGGTGGATTTGGGTTTC TTCGGCGAAGAAACCAGGCACCCAAATCCCACCTTGACGCCAACCCCTTTGCCGGTTCAGAAATCCTTACCCGTCCTTGAAA ATGCCAACCCCTCTACTTTCCCTCCCAATAGGCAAATGCAAACGCCGCCGGAGGACGATCGGAGCCCTGTCCCATTTCCTGTC CAGAAGCCTGTAGTAGCCCACGAAGAGCTCCCCTCCTTTCCACTTGCGCAGAACACTGCCCTCTTAGAAGGATCCTTATCATT TGTGCATAAAACTGGGGTTTTCGATGATAAGTCTAGTGCCTTGCCGTATTCTGCAGCTCAGAAGCCTTCCCAAAATCACAATTC TTTGTTGTACCCTGCTGCTACTGCTCAGAAACCGTCCCAAAATCATAATCCTCTGTTGTACCCTACTGCTGCTGCTCAGAAGCT TCCCCAAAATCACAATCCTTTGTTGTATCCTGCTGCTGCGTCTCAAGAGCCTGCTCAAAATCATACTTCTTTGCTGTACCCTGAT GCTGCTAAGAAGCCTGCCCAAAATCACAATTCTTTGTTGTATTCTGCTATTCAGAAGCCTGCTGCTGTCTGTGACGACAATCAC AATTATATCCCATATTTGACTTCTCTGAAACCCATTGTCGATAGTGGGTCTTCGCAGTTTCCAAATCACACTACTGTGCAAGTTC TAGAACAGAACGGCAATGCATCACCATTCGCTGTTCAGAAGCCTATGGAGAACAAAGGGGTTTTTATACCAAAGGAGGAAG TGGACAATTCAAAACCCATGTTTCACTTTACAAATGGCAGTCTCCAGCGGGAAACTCTTGCAAACAGAGAATCTCAGAACCA AATGGAAGAGAAGATGAAAAAGCAAAAGACCGTTGATGAGAAGCATGGTTCCTTGCCTTCAATGTCTTCTGGTGCTTTCTTT GGTGGTGTTCGATCTGGTGCTGAGTCAGAGCATTCTGATGTTGAAGCTGCATCCTTCAAGGAAATGGAAGAAGCTGTTGTCGA GAAGAAGCCTCGGAAAAGAGGAAGGAAGCCTGCAAATGGCCGAGAAGAACCCTTGAATCATGTTGAAGCTGAACGCCAGC GGCGTGAGAAGCTGAATCAGAGGTTCTATGCACTTCGAGCAGTAGTGCCAAATGTGTCAAAGATGGATAAAGCTTCATTGCT TGGTGATGCTGTGTCGTATATTAATGAACTTAGAAGCAAAGTTCAAGAACTTGAGACTGAAAGGAAGGAGCTTGAATCCCAT GTTGAAGCTACCAAAAAGGAATTGCTATCGTCTCACTCTGGATTCTCTGGTGCCAACTTTGGGTTCGCTAAGGATCTGTCCGGT TCTTCTCAGGTGCCTGATAGCAAAGGATTTGGCACAAAGCAGTGCCCTGGATTAGATTTGGATGTCCGAGTTCTTGGTGCAGA AGCCATGGTCAGAATCCAGTCTGGTAAAAAGAATCATCCTGCGGCCAGATTGATGACAGCTTTTCAAGAGCTTGAACTTGAG GTTCATCATGCTAGTGTCTCAACTGTCAATGAGTTAATGCTTCAAAATGTTATTGTCAGGTTGCCAAAAAGTCTGTACACAGAA GAACAACTCAGCGCTGCCCTTTTTAAAAAGCTATCAAATCCAGGATTTTGTTAA

$>$ MYC4

ATGGCGCGGCAAAGTATCTGGATCAGCGATAATTTGGAAAGCAATTCATTAGTCGAGGCGTTCATGGCCTCCTCCTCTTCGTCT TCCTCTGCCCCCAATTTAAGGATGGATCCGAATTTGACCCTGGAGACATTGCAGCAGCGCTTGCAAATTCTGGTCGAGACTGC CTCAATTGTGTGGACATATGCAATATTTTGGCAGGTTTCATGTGATGTTAATGGTGCCCCTCAGCTGTGCTGGGGGGATGGGTA CTATAAGGGGCCCAAGAATGCAGAGGAAGATGAACAACTCAGAATGCGCAGCCGTATGACCATAACCCCAGCCGACCAGG AGCTCAGGAAGAAGGTTTTGAGAGATTTACATTCCATGATCAGTGGAGCTGATGAGACAAATCAACAGGACGAGGACGTTA CAGATGCCGAATGGTTTTATTTGGTTTCTATGATGCAGACCTTTTTGTTAGGGTTCGGAGTCCCAGGATTTGCATTTTCTAGAGG AGCTCATGCTTGGTTAGTCGGAGCGGAGAGACTGCAGACATCGAATTGTGACAGGGCAAAACAAGCTCAACAACTTGGGAT TCAAACCCTGGTTTGTATTCCCATTCAGGGCGGGGTGGTGGAATTTGGATCCACGGACCTCATTCCTGAGAACTGGTTATTTCT GCAGCAGGTTAACCGCTCATTTAACCTTAACTTCAACCAGACTTATGCGAATCCGGTTCAAAACCAGTCATTATGGTTGGCTG AACCAGCGAGTTTGACACATGGCAATTCCTTTCAGTCCGCACAATATATTGAGTCAGCCAAAACAGTTGTTAGTAATGCTGAA GTCCAGTCTTTGGACTCTGCACTTCCTCAGGAATTGGAATTTACAAACTTTGGTTCAGTCACAGACAGACATAAATTATCTCTA TTTGACGACCAGGGATCTTTATCTGTTAGAGGCGATCAATCATTGATGCATCATCAAGTTCAGAAGACCGAGGTCTTTGAAGC ACCGACAGTCCACATTATGGAAACAATGAATGGTGTGGAACAAAATGGTAGTGCTGTGGGCTTGCTTGCATTAAAACCAACC AGGTCTGTAGATACCACTAGTTTGCCAAAACAAAATGGGAGTGCTGGGGGCGTCAATGGTGCTGAAAAGAATGCATCAAAA AGAATGAGAACTGAAGATACCCTTCATTTGCCATCTAATCCTTCTGGAATAGCTGTAGGTAGTTTCCGTTCCAGTATTGAATCA GAGCTTTCTGATGTAGAGCCCTCTGCATCAATTAAAGAATCAGAGTCTGTTGTAGTGGAAAAAAAGCCACGGAAACGTGGGA GGAAACCTGCAAATGGCCGTGAAGAGCCTCTGAATCATGTGGAGGCTGAACGCCAAAGGCGAGAGAAACTAAACCAGAAA TTCTACGAGCTTCGTGCTGTGGTTCCAAATGTCTCAAAGATGGACAAGGCTTCTTTGCTTGCAGATGCTGTTACTTATATCAAC GATCTTAGCTCCAGGCAACAAAGTTTGGAGCTTGAGAGGGATGAATTACGAACTCAAATTGGTGCTGCAAAAAAGGAATTGT TGGTACTTCCTTCCAAGTTTGGAAATAAAGAGCCATCAGGTCATACAAATATGGACCTAAAGGGTTCTAGTGGGGGGAAATTC CCTGGCTTGGATTCAGAAGTTCGCATTCTTGGCCAAGAGGCAATGATAAAAATTCAGTGTGCTAAACACAACCATCCTGTCGC TAGATTGATGACTGCACTGCAAGAACTTGAAATGGAAGTCCTCCATGCAAGTATTTCTACCATAAAAGACGCTCTGATGATTC AGACAGTGATTGCTAAAATGACCAGAGTTTTGTATACAGAACAACAACTTCATGCCCTGCTTTGCAAGAAAGTAGCTAATCTG TAG

$>$ JAM1

ATGGCGCGGCAAAGTATCTGGATCAGCGATAATTTGGAAAGCAATTCATTAGTCGAGGCGTTCATGGCCTCCTCCTCTTCGTCT TCCTCTGCCCCCAATTTAAGGATGGATCCGAATTTGACCCAGGAGCTCAGGAAGAAGGTTTTGAGAGATTTACATTCCATGAT 
CAGTGGAGCTGATGAGACAAATCAACAGGACGAGGACGTTACAGATGCCGAATGGTTTTATTTGGTTTCTATGATGCAGACCT TTTTGTTAGGGTTCGGAGTCCCAGGATTTGCATTTTCTAGAGGAGCTCATGCTTGGTTAGTCGGAGCGGAGAGACTGCAGACA TCGAATTGTGACAGGGCAAAACAAGCTCAACAACTTGGGATTCAAACCCTGGTTTGTATTCCCATTCAGGGCGGGGTGGTGG AATTTGGATCCACGGACCTCATTCCTGAGAACTGGTTATTTCTGCAGCAGGTTAACCGCTCATTTAACCTTAACTTCAACCAGA CTTATGCGAATCCGGTTCAAAACCAGTCATTATGGTTGGCTGAACCAGCGAGTTTGACACATGGCAATTCCTTTCAGTCCGCA CAATATATTGAGTCAGCCAAAACAGTTGTTAGTAATGCTGAAGTCCAGTCTTTGGACTCTGCACTTCCTCAGGAATTGGAATTT ACAAACTTTGGTTCAGTCACAGACAGACATAAATTATCTCTATTTGACGACCAGGGATCTTTATCTGTTAGAGGCGATCAATCA TTGATGCATCATCAAGTTCAGAAGACCGAGGTCTTTGAAGCACCGACAGTCCACATTATGGAAACAATGAATGGTGTGGAAC AAAATGGTAGTGCTGTGGGCTTGCTTGCATTAAAACCAACCAGGTCTGTAGATACCACTAGTTTGCCAAAACAAAATGGGAG TGCTGGGGGCGTCAATGGTGCTGAAAAGAATGCATCAAAAAGAATGAGAACTGAAGATACCCTTCATTTGCCATCTAATCCT TCTGGAATAGCTGTAGGTAGTTTCCGTTCCAGTATTGAATCAGAGCTTTCTGATGTAGAGCCCTCTGCATCAATTAAAGAATCA GAGTCTGTTGTAGTGGAAAAAAAGCCACGGAAACGTGGGAGGAAACCTGCAAATGGCCGTGAAGAGCCTCTGAATCATGTG GAGGCTGAACGCCAAAGGCGAGAGAAACTAAACCAGAAATTCTACGAGCTTCGTGCTGTGGTTCCAAATGTCTCAAAGATG GACAAGGCTTCTTTGCTTGCAGATGCTGTTACTTATATCAACGATCTTAGCTCCAGGCAACAAAGTTTGGAGCTTGAGAGGGA TGAATTACGAACTCAAATTGGTGCTGCAAAAAAGGAATTGTTGGTACTTCCTTCCAAGTTTGGAAATAAAGAGCCATCAGGT CATACAAATATGGACCTAAAGGGTTCTAGTGGGGGGAAATTCCCTGGCTTGGATTCAGAAGTTCGCATTCTTGGCCAAGAGGC AATGATAAAAATTCAGTGTGCTAAACACAACCATCCTGTCGCTAGATTGATGACTGCACTGCAAGAACTTGAAATGGAAGTC CTCCATGCAAGTATTTCTACCATAAAAGACGCTCTGATGATTCAGACAGTGATTGCTAAAATGACCAGAGTTTTGTATACAGA ACAACAACTTCATGCCCTGCTTTGCAAGAAAGTAGCTAATCTGTAG

$>$ JAM2

ATGGCGAGCGAGGCTGGTGCCATACTGAGGTTCTGGAACGAGGAAGATAAGGCTTTGGTGAAATCACTTATAGGTCCCAGTG CCTTTGATCACTTGATGTTGAGCTATGTTTCTTCTGAAGGGCTCGTGTCTGGGATCAGTGATTGTGCATTGCAGCAGAAGCTTC AAAACTTAGTTGAATCTTCGTCAATAAACTGGACTTATGCTATTTTCTGGCAGCTTTCAAGATCGAAAACTGGAGAGATGGTT CTTGGATGGGGTGATGGCCACTTCAAGGGGCCTCGGGAGGGTCAGGAAGCTGACCAGGCAAGAGGTTTTGATCAAAGGTTT ATGGAAACGGATCAGCTCAAGAAAAAGGTATTGCAGAAACTCCAGACCTTCTTTGGGGGTGGGTGTGAAGAGGATAATAATT TTATTGCAGGATTAGAGAATGTTTCTGATACAGAGATGTTTTATTTGACGTCTATGTACTATTCTTTTCCTCGAGGAATTGGTATT CCTGGCCAGACCCTTGCCAGTGGAAAATGTATCTGGATGAATGAACTTAGCAAATTACCTACAAATTTTTGCACCAGATCATA TCTTGCCAAAACAGGAGGCATTCAGACCCTTGTCTGTGTGCCAATGGAAAATGGCATTCTGGAAGTTGGGTCTGTGGAGACG ATCAGAGAAAGCAAAGATGCTGTAGATAAGATTAAATCTAGCTTCAAGGAAAATGTCAGTGATGAAATTCGACCTCGACAAA CCGTGAAGGTTTCTCCATTTTTGCCCTTTTCACCAAATCCAATCAGGGCGAATCCTATTAATGCCAAAATTCCTCCAGCTGTTA AGCCCTCTGTAGATTGGAAGATTTTTGGCCATGAACTAATCAAATCTTCAGAATCTGTGGCCACCAAAGTGGAAGACAGAGA TCGCCATTACCGCACAGCCTTTGGGTCGCCATACCCTCATGCACCTTATATCACAAATGAGCAAAGGATATCTTATGCAACTAC TAATCAGAATGGCTTGCATTCAGCAAATTGGAATCATGCCAGTAATGGTGAGGCTGGAGAGATTTATAATACCCATGATATAAT TAAACCGTCTGGTAGACTTTCATCTCTTAGTGTAGCAGGGTCTTCTCTAAGTGTTGTGTCTGCTCGACCAAGTATGATGGAGTC TGAAGACCATTCGGATGTTGAGGCTTCCTGTAGAGAGGATCGTAGGCCTGTGGTGGTTGAAGAGCGAAGACCAAGGAAAAG GGGAAGGAAACCAGCTAATGGAAGAGAAGAGCCACTCAATCATGTTGAAGCAGAACGTCAACGGCGCGAGAAACTTAACC AACGGTTTTATGCATTGCGAGCTGTGGTGCCCAACATCTCAAAAATGGATAAAGCTTCACTTCTAGGTGATGCGATTTCCTACA TTCAAGAACTGCAGAGTAAGGTGAAGGATATGGAAATGGAAAAGGAGCAGCAGCAGCAGCAGTTGAAGCCCAGTGTGCAA GAAGGCAGAAGTTTAGATACCATTTCTAACATAGATGTTCAGATGATGAGTGGAGAAGCTACTGTGAGGGTTAGCTGTCCAA AGGAAAGTCATCCAGTTGGTAGGGTAATGGTTGCGTTGCAAAGAATGCAATTGGACGTAAATCATGCAAACATTTCTGCAGCT AATGAAAATATCCTTCATACGTTTGTGATTAAGCTTGGGGCATCCCAGGCATTGACTAGAGAACAGTTACTGGAGGCAATTTC TGAATGGACCCCCAAACAAGGACAATGA

$>$ MYC2YL

ATGATGGGGAACGAGGGGACAGCGTCAATGCGTTTGTGGAACGAGGACAACAATGCCCTGATTGAGGCGTTCATGGGGCAC ATGGACTACTCTTTCCCCTGGAATGGCATTGACGCCAATCCCTCGCCTGCACCAGCACCAGCACCAGCACCAGCCCCTATTTT GACAGCCCCTCCGCCGCAATCGTCATTCAACCAAGACACACTGCAGCAGCGCTTGCAGGCTTTGGTGGAAGGCGCTTCAGC AAGCTGGACATACGCCATTTTCTGGCAGATTTCCTCCGACCCAGAGAACGCAATGGTCTTAGGCgctGGCgctGGCTACgctAAG GGTCCTCGAGATTTGACGGACGAGGAAAGTGCGTCAAAGAGGGTTTCTGCTTCTTCCTCCGCTTTTGAAGCGACGGCTTCGG ATCAGGAGCTCCGCAAGAAAGTGCTTCGAGATCTCCACACGCTCATCAACCCCGACATTGAAATGACTGATATTTCATCGAC AGTTGACGGAGAAGTAACGGACGAGGAGTGGTTTTATCTGGTGTCAATGATGCAGTCGTTTGTCAATGGATGCGGCGTGCCT GGGCAGGCGTTTTTTACTGCAACGCCCATTTGGATTACAGGCCCCGAGACACTGCAGGCTTATAACTGTGACAGGGCCCGTC AGGCCCAGCAATTTGGAATTCGGACTTTGGTATGCATTCCGTCGCCCAACGGAGTTGTGGAGTTGGGTTCCACGGATTTGATT ACACAGAACTGGAATTTGATGCAGCAAGCAAGGAATTCATTCACTTTTAACGACAACCCAAATCCTCTATGGGAAGAAGGG AACCCTAGCTATAACAACAACAATAGCGTCGACGCTGATCCGTCGCTTTGGTTGACGGAGCCGCCTCACGTTGAAGAGCAGC CGCAGGCAGTAGATGCGGAGAAGCCCAAACAACATACAGAAACCGAGGGCGTTAACGGGTTTTTTGAACTGGGGTTTTCGG ACCTAGGGTTTGGGGTTGATCGTCAATTGACGGGAGCATTTGAAGACCGTCAATCGACTGGAATTTTACAACCAGGTACACA GATGGTGTACCCGTCCTACAAATCAGAGGCCGTAGAGAGCACTCAGGCTTTGCAGAATGGAAATAGACAGCAAGTGGCTTTG GATGGGGATGGCGATGGGGATGGGGATGTAATCACTCTGAGCTTTGCCGAAGCTCGAAACGATGACCCTGCACAGAGCTTAG TGGCTTCAGCTACGGCTGCTGCTGTTTTGCCGATGAAGTCTAGTGTTGAATCAGAGCACTCTGACATTGAGGCCTCCATTAAG GAGCCGGAGTGCAGCCAGGCTACTTTTGTGGAGAGGAAGCCCAGAAAAAGAGGGAGAAAGCCTGCAAATGGCAGGGAGG AGCCACTGAATCATGTGGAGGCTGAGAGGCAGAGGAGGGAGAAGTTAAACCAGAGGTTCTATGCACTCAGGGCTGTGGTTC CCAATGTGTCCAAGATGGATAAGGCATCTTTGTTGGGCGATGCTATTGCTTATATTAATGAATTACGAAGCAAGGTGGTGGATG CAGAGACTCATAAGAAGGAGCTGCAGGTCCAAGTTGAGGCATTGAAGAAGGAATTGGTGGTGGTTAGAGAATCTGGTGCTT CTGGGCCTAATTTTGGCTTGATCAAGGATCACTACCCCACAGCTGATTCATCTGATGTCAAAGGCCATGGCTTGAATAACAGT AAATGTCATGGTATAGAGCTCGAGGTTCGATTACTTGGCAGAGAGGCAATGATCAGAGTTCAGAGCCCAAAACAGAACCAC CCTGTTGCAAGGTTGATGGGGGCATTGAAAGAGCTCGATCTTGAGGTCCACCATGCCAGTGTATCAACGGTGAAGGAGCTGA TGATTCAAACAGTTATTGTGAAGATGACAGGAGGTATTGTATATAGTCAAGAACAACTAAATGCAGCACTAAGCAAGTCAGTT GCAGATTCTGGCCTTAGGTAA

$>$ MYC2D

ATGATGGGGAACGAGGGGACAGCGTCAATGCGTTTGTGGAACGAGGACAACAATGCCCTGATTGAGGCGTTCATGGGGCAC ATGGACTACTCTTTCCCCTGGAATGGCATTGACGCCAATCCCTCGCCTGCACCAGCACCAGCACCAGCACCAGCCCCTATTTT GACAGCCCCTCCGCCGCAATCGTCATTCAACCAAGACACACTGCAGCAGCGCTTGCAGGCTTTGGTGGAAGGCGCTTCAGC AAGCTGGACATACGCCATTTTCTGGCAGATTTCCTCCGACCCAGAGAACGCAATGGTCTTAGGCTGGGGCaacGGCTACTACA AGGGTCCTCGAGATTTGACGGACGAGGAAAGTGCGTCAAAGAGGGTTTCTGCTTCTTCCTCCGCTTTTGAAGCGACGGCTTC GGATCAGGAGCTCCGCAAGAAAGTGCTTCGAGATCTCCACACGCTCATCAACCCCGACATTGAAATGACTGATATTTCATCG ACAGTTGACGGAGAAGTAACGGACGAGGAGTGGTTTTATCTGGTGTCAATGATGCAGTCGTTTGTCAATGGATGCGGCGTGC CTGGGCAGGCGTTTTTTACTGCAACGCCCATTTGGATTACAGGCCCCGAGACACTGCAGGCTTATAACTGTGACAGGGCCCGT CAGGCCCAGCAATTTGGAATTCGGACTTTGGTATGCATTCCGTCGCCCAACGGAGTTGTGGAGTTGGGTTCCACGGATTTGAT TACACAGAACTGGAATTTGATGCAGCAAGCAAGGAATTCATTCACTTTTAACGACAACCCAAATCCTCTATGGGAAGAAGGG AACCCTAGCTATAACAACAACAATAGCGTCGACGCTGATCCGTCGCTTTGGTTGACGGAGCCGCCTCACGTTGAAGAGCAGC CGCAGGCAGTAGATGCGGAGAAGCCCAAACAACATACAGAAACCGAGGGCGTTAACGGGTTTTTTGAACTGGGGTTTTCGG ACCTAGGGTTTGGGGTTGATCGTCAATTGACGGGAGCATTTGAAGACCGTCAATCGACTGGAATTTTACAACCAGGTACACA GATGGTGTACCCGTCCTACAAATCAGAGGCCGTAGAGAGCACTCAGGCTTTGCAGAATGGAAATAGACAGCAAGTGGCTTTG GATGGGGATGGCGATGGGGATGGGGATGTAATCACTCTGAGCTTTGCCGAAGCTCGAAACGATGACCCTGCACAGAGCTTAG TGGCTTCAGCTACGGCTGCTGCTGTTTTGCCGATGAAGTCTAGTGTTGAATCAGAGCACTCTGACATTGAGGCCTCCATTAAG GAGCCGGAGTGCAGCCAGGCTACTTTTGTGGAGAGGAAGCCCAGAAAAAGAGGGAGAAAGCCTGCAAATGGCAGGGAGG AGCCACTGAATCATGTGGAGGCTGAGAGGCAGAGGAGGGAGAAGTTAAACCAGAGGTTCTATGCACTCAGGGCTGTGGTTC CCAATGTGTCCAAGATGGATAAGGCATCTTTGTTGGGCGATGCTATTGCTTATATTAATGAATTACGAAGCAAGGTGGTGGATG CAGAGACTCATAAGAAGGAGCTGCAGGTCCAAGTTGAGGCATTGAAGAAGGAATTGGTGGTGGTTAGAGAATCTGGTGCTT 
CTGGGCCTAATTTTGGCTTGATCAAGGATCACTACCCCACAGCTGATTCATCTGATGTCAAAGGCCATGGCTTGAATAACAGT AAATGTCATGGTATAGAGCTCGAGGTTCGATTACTTGGCAGAGAGGCAATGATCAGAGTTCAGAGCCCAAAACAGAACCAC CCTGTTGCAAGGTTGATGGGGGCATTGAAAGAGCTCGATCTTGAGGTCCACCATGCCAGTGTATCAACGGTGAAGGAGCTGA TGATTCAAACAGTTATTGTGAAGATGACAGGAGGTATTGTATATAGTCAAGAACAACTAAATGCAGCACTAAGCAAGTCAGTT GCAGATTCTGGCCTTAGGTAA $>$ MYC2WDY

ATGATGGGGAACGAGGGGACAGCGTCAATGCGTTTGTGGAACGAGGACAACAATGCCCTGATTGAGGCGTTCATGGGGCAC ATGGACTACTCTTTCCCCTGGAATGGCATTGACGCCAATCCCTCGCCTGCACCAGCACCAGCACCAGCACCAGCCCCTATTTT GACAGCCCCTCCGCCGCAATCGTCATTCAACCAAGACACACTGCAGCAGCGCTTGCAGGCTTTGGTGGAAGGCGCTTCAGC AAGCTGGACATACGCCATTTTCTGGCAGATTTCCTCCGACCCAGAGAACGCAATGGTCTTAGGCTGGGGCGACGGCTACTAC AAGGGTCCTCGAGATTTGACGGACGAGGAAAGTGCGTCAAAGAGGGTTTCTGCTTCTTCCTCCGCTTTTGAAGCGACGGCTT CGGATCAGGAGCTCCGCAAGAAAGTGCTTCGAGATCTCCACACGCTCATCAACCCCGACATTGAAATGACTGATATTTCATC GACAGTTGACGGAGAAGTAACGGACGAGGAGTGGTTTgctgctGTGTCAATGATGCAGTCGTTTGTCAATGGATGCGGCGTGCC TGGGCAGGCGTTTTTTACTGCAACGCCCATTTGGATTACAGGCCCCGAGACACTGCAGGCTTATAACTGTGACAGGGCCCGTC AGGCCCAGCAATTTGGAATTCGGACTTTGGTATGCATTCCGTCGCCCAACGGAGTTGTGGAGTTGGGTTCCACGGATTTGATT ACACAGAACTGGAATTTGATGCAGCAAGCAAGGAATTCATTCACTTTTAACGACAACCCAAATCCTCTATGGGAAGAAGGG AACCCTAGCTATAACAACAACAATAGCGTCGACGCTGATCCGTCGCTTTGGTTGACGGAGCCGCCTCACGTTGAAGAGCAGC CGCAGGCAGTAGATGCGGAGAAGCCCAAACAACATACAGAAACCGAGGGCGTTAACGGGTTTTTTGAACTGGGGTTTTCGG ACCTAGGGTTTGGGGTTGATCGTCAATTGACGGGAGCATTTGAAGACCGTCAATCGACTGGAATTTTACAACCAGGTACACA GATGGTGTACCCGTCCTACAAATCAGAGGCCGTAGAGAGCACTCAGGCTTTGCAGAATGGAAATAGACAGCAAGTGGCTTTG GATGGGGATGGCGATGGGGATGGGGATGTAATCACTCTGAGCTTTGCCGAAGCTCGAAACGATGACCCTGCACAGAGCTTAG TGGCTTCAGCTACGGCTGCTGCTGTTTTGCCGATGAAGTCTAGTGTTGAATCAGAGCACTCTGACATTGAGGCCTCCATTAAG GAGCCGGAGTGCAGCCAGGCTACTTTTGTGGAGAGGAAGCCCAGAAAAAGAGGGAGAAAGCCTGCAAATGGCAGGGAGG AGCCACTGAATCATGTGGAGGCTGAGAGGCAGAGGAGGGAGAAGTTAAACCAGAGGTTCTATGCACTCAGGGCTGTGGTTC CCAATGTGTCCAAGATGGATAAGGCATCTTTGTTGGGCGATGCTATTGCTTATATTAATGAATTACGAAGCAAGGTGGTGGATG CAGAGACTCATAAGAAGGAGCTGCAGGTCCAAGTTGAGGCATTGAAGAAGGAATTGGTGGTGGTTAGAGAATCTGGTGCTT CTGGGCCTAATTTTGGCTTGATCAAGGATCACTACCCCACAGCTGATTCATCTGATGTCAAAGGCCATGGCTTGAATAACAGT AAATGTCATGGTATAGAGCTCGAGGTTCGATTACTTGGCAGAGAGGCAATGATCAGAGTTCAGAGCCCAAAACAGAACCAC CCTGTTGCAAGGTTGATGGGGGCATTGAAAGAGCTCGATCTTGAGGTCCACCATGCCAGTGTATCAACGGTGAAGGAGCTGA TGATTCAAACAGTTATTGTGAAGATGACAGGAGGTATTGTATATAGTCAAGAACAACTAAATGCAGCACTAAGCAAGTCAGTT GCAGATTCTGGCCTTAGGTAA

$>$ MYC3YL

ATGGCTCTGGCGGGAATGAAGTTGTGGAGTGACGATGCCATGGTGGAGGCGTTCATGGCGTCCTCCTCTCCGCAGGTGGGAG TTGGGTGGAGCAATGGCGGCGCCACTGCGACTGTGTCTCCGTGTAACGACAATTCCTTGTTTAATCAGGAGACGCTACAGCA GAGGCTTCAGATGTTAATTGAGGGCGCCCAAGAGAGTTGGACATACGCCATATTCTGGCAGTGGTCGTACGACCCAAGCGGC GCCATCGTTTTCTGCgcgGCCgcgGGCTACgcgAAAGGCGGCGGCGCCGCCGAGGAGGAGAAGCAAAGGAGCGCAGTTGAGCA GGAGGTGAGGAAAAAGGTGTTGCGGGAGCTGCACGCGCTCATTAACGGCGGCGGCACCGAAGCAGATCCCCCGGCTGAGG CGGAAGCGGAAGCGGATGAGGAGGTGACTGATGCGGAGTGGTTTTATTTGGTGTCGATGACGCACTCGTTTGGCGGCGGGCA GGGTGTGGCGGGGCAGGCGTATCTCAGCGGTTTGCCGGCTTGGCTGTCTGGGTCGCAGAAAATGGAGGGGTGTCATTGCGAG AGAGCTAAACAGGCGGCTCAGTTCGGCATTCAAACCATGGTATGTATTCCTACTCCCAATGGCGTTGTGGAATTGGGGTCTGT GGTTTTGCTTGCTCACAATTGGGAGCTGGTTCAACAGGCTAAGACCTCTTTTACTTTTTCGTCCTTTGGCTTGTGGGAAGATCA TGGAAATAATAACAACACTACATCTTCCGACAACAATACCAATCTCATCATCAACAACAACAACGACAACAACTTTTGTAAC AATAACCAGTCTCTGTGGGTTCCGGGTTCCCCTTTTTTCGGTGGCCATGCCCAGGAATCTCCCCTGGTGGATTTGGGTTTCTTC GGCGAAGAAACCAGGCACCCAAATCCCACCTTGACGCCAACCCCTTTGCCGGTTCAGAAATCCTTACCCGTCCTTGAAAATG CCAACCCCTCTACTTTCCCTCCCAATAGGCAAATGCAAACGCCGCCGGAGGACGATCGGAGCCCTGTCCCATTTCCTGTCCA GAAGCCTGTAGTAGCCCACGAAGAGCTCCCCTCCTTTCCACTTGCGCAGAACACTGCCCTCTTAGAAGGATCCTTATCATTTG TGCATAAAACTGGGGTTTTCGATGATAAGTCTAGTGCCTTGCCGTATTCTGCAGCTCAGAAGCCTTCCCAAAATCACAATTCTT TGTTGTACCCTGCTGCTACTGCTCAGAAACCGTCCCAAAATCATAATCCTCTGTTGTACCCTACTGCTGCTGCTCAGAAGCTTC CCCAAAATCACAATCCTTTGTTGTATCCTGCTGCTGCGTCTCAAGAGCCTGCTCAAAATCATACTTCTTTGCTGTACCCTGATG CTGCTAAGAAGCCTGCCCAAAATCACAATTCTTTGTTGTATTCTGCTATTCAGAAGCCTGCTGCTGTCTGTGACGACAATCACA ATTATATCCCATATTTGACTTCTCTGAAACCCATTGTCGATAGTGGGTCTTCGCAGTTTCCAAATCACACTACTGTGCAAGTTCT AGAACAGAACGGCAATGCATCACCATTCGCTGTTCAGAAGCCTATGGAGAACAAAGGGGTTTTTATACCAAAGGAGGAAGT GGACAATTCAAAACCCATGTTTCACTTTACAAATGGCAGTCTCCAGCGGGAAACTCTTGCAAACAGAGAATCTCAGAACCA AATGGAAGAGAAGATGAAAAAGCAAAAGACCGTTGATGAGAAGCATGGTTCCTTGCCTTCAATGTCTTCTGGTGCTTTCTTT GGTGGTGTTCGATCTGGTGCTGAGTCAGAGCATTCTGATGTTGAAGCTGCATCCTTCAAGGAAATGGAAGAAGCTGTTGTCGA GAAGAAGCCTCGGAAAAGAGGAAGGAAGCCTGCAAATGGCCGAGAAGAACCCTTGAATCATGTTGAAGCTGAACGCCAGC GGCGTGAGAAGCTGAATCAGAGGTTCTATGCACTTCGAGCAGTAGTGCCAAATGTGTCAAAGATGGATAAAGCTTCATTGCT TGGTGATGCTGTGTCGTATATTAATGAACTTAGAAGCAAAGTTCAAGAACTTGAGACTGAAAGGAAGGAGCTTGAATCCCAT GTTGAAGCTACCAAAAAGGAATTGCTATCGTCTCACTCTGGATTCTCTGGTGCCAACTTTGGGTTCGCTAAGGATCTGTCCGGT TCTTCTCAGGTGCCTGATAGCAAAGGATTTGGCACAAAGCAGTGCCCTGGATTAGATTTGGATGTCCGAGTTCTTGGTGCAGA AGCCATGGTCAGAATCCAGTCTGGTAAAAAGAATCATCCTGCGGCCAGATTGATGACAGCTTTTCAAGAGCTTGAACTTGAG GTTCATCATGCTAGTGTCTCAACTGTCAATGAGTTAATGCTTCAAAATGTTATTGTCAGGTTGCCAAAAAGTCTGTACACAGAA GAACAACTCAGCGCTGCCCTTTTTAAAAAGCTATCAAATCCAGGATTTTGTTAA $>$ MYC3D

ATGGCTCTGGCGGGAATGAAGTTGTGGAGTGACGATGCCATGGTGGAGGCGTTCATGGCGTCCTCCTCTCCGCAGGTGGGAG TTGGGTGGAGCAATGGCGGCGCCACTGCGACTGTGTCTCCGTGTAACGACAATTCCTTGTTTAATCAGGAGACGCTACAGCA GAGGCTTCAGATGTTAATTGAGGGCGCCCAAGAGAGTTGGACATACGCCATATTCTGGCAGTGGTCGTACGACCCAAGCGGC GCCATCGTTTTCTGCTGGGCCaacGGCTACTTCAAAGGCGGCGGCGCCGCCGAGGAGGAGAAGCAAAGGAGCGCAGTTGAGC AGGAGGTGAGGAAAAAGGTGTTGCGGGAGCTGCACGCGCTCATTAACGGCGGCGGCACCGAAGCAGATCCCCCGGCTGAG GCGGAAGCGGAAGCGGATGAGGAGGTGACTGATGCGGAGTGGTTTTATTTGGTGTCGATGACGCACTCGTTTGGCGGCGGGC AGGGTGTGGCGGGGCAGGCGTATCTCAGCGGTTTGCCGGCTTGGCTGTCTGGGTCGCAGAAAATGGAGGGGTGTCATTGCGA GAGAGCTAAACAGGCGGCTCAGTTCGGCATTCAAACCATGGTATGTATTCCTACTCCCAATGGCGTTGTGGAATTGGGGTCTG TGGTTTTGCTTGCTCACAATTGGGAGCTGGTTCAACAGGCTAAGACCTCTTTTACTTTTTCGTCCTTTGGCTTGTGGGAAGATC ATGGAAATAATAACAACACTACATCTTCCGACAACAATACCAATCTCATCATCAACAACAACAACGACAACAACTTTTGTAA CAATAACCAGTCTCTGTGGGTTCCGGGTTCCCCTTTTTTCGGTGGCCATGCCCAGGAATCTCCCCTGGTGGATTTGGGTTTCTT CGGCGAAGAAACCAGGCACCCAAATCCCACCTTGACGCCAACCCCTTTGCCGGTTCAGAAATCCTTACCCGTCCTTGAAAAT GCCAACCСCTCTACTTTCCCTCCCAATAGGCAAATGCAAACGCCGCCGGAGGACGATCGGAGCCCTGTCCCATTTCCTGTCC AGAAGCCTGTAGTAGCCCACGAAGAGCTCCCCTCCTTTCCACTTGCGCAGAACACTGCCCTCTTAGAAGGATCCTTATCATTT GTGCATAAAACTGGGGTTTTCGATGATAAGTCTAGTGCCTTGCCGTATTCTGCAGCTCAGAAGCCTTCCCAAAATCACAATTCT TTGTTGTACCCTGCTGCTACTGCTCAGAAACCGTCCCAAAATCATAATCCTCTGTTGTACCCTACTGCTGCTGCTCAGAAGCTT CCCCAAAATCACAATCCTTTGTTGTATCCTGCTGCTGCGTCTCAAGAGCCTGCTCAAAATCATACTTCTTTGCTGTACCCTGAT GCTGCTAAGAAGCCTGCCCAAAATCACAATTCTTTGTTGTATTCTGCTATTCAGAAGCCTGCTGCTGTCTGTGACGACAATCAC AATTATATCCCATATTTGACTTCTCTGAAACCCATTGTCGATAGTGGGTCTTCGCAGTTTCCAAATCACACTACTGTGCAAGTTC TAGAACAGAACGGCAATGCATCACCATTCGCTGTTCAGAAGCCTATGGAGAACAAAGGGGTTTTTATACCAAAGGAGGAAG TGGACAATTCAAAACCCATGTTTCACTTTACAAATGGCAGTCTCCAGCGGGAAACTCTTGCAAACAGAGAATCTCAGAACCA AATGGAAGAGAAGATGAAAAAGCAAAAGACCGTTGATGAGAAGCATGGTTCCTTGCCTTCAATGTCTTCTGGTGCTTTCTTT GGTGGTGTTCGATCTGGTGCTGAGTCAGAGCATTCTGATGTTGAAGCTGCATCCTTCAAGGAAATGGAAGAAGCTGTTGTCGA GAAGAAGCCTCGGAAAAGAGGAAGGAAGCCTGCAAATGGCCGAGAAGAACCCTTGAATCATGTTGAAGCTGAACGCCAGC GGCGTGAGAAGCTGAATCAGAGGTTCTATGCACTTCGAGCAGTAGTGCCAAATGTGTCAAAGATGGATAAAGCTTCATTGCT 
TGGTGATGCTGTGTCGTATATTAATGAACTTAGAAGCAAAGTTCAAGAACTTGAGACTGAAAGGAAGGAGCTTGAATCCCAT GTTGAAGCTACCAAAAAGGAATTGCTATCGTCTCACTCTGGATTCTCTGGTGCCAACTTTGGGTTCGCTAAGGATCTGTCCGGT TCTTCTCAGGTGCCTGATAGCAAAGGATTTGGCACAAAGCAGTGCCCTGGATTAGATTTGGATGTCCGAGTTCTTGGTGCAGA AGCCATGGTCAGAATCCAGTCTGGTAAAAAGAATCATCCTGCGGCCAGATTGATGACAGCTTTTCAAGAGCTTGAACTTGAG GTTCATCATGCTAGTGTCTCAACTGTCAATGAGTTAATGCTTCAAAATGTTATTGTCAGGTTGCCAAAAAGTCTGTACACAGAA GAACAACTCAGCGCTGCCCTTTTTAAAAAGCTATCAAATCCAGGATTTTGTTAA $>$ MYC3WDY

ATGGCTCTGGCGGGAATGAAGTTGTGGAGTGACGATGCCATGGTGGAGGCGTTCATGGCGTCCTCCTCTCCGCAGGTGGGAG TTGGGTGGAGCAATGGCGGCGCCACTGCGACTGTGTCTCCGTGTAACGACAATTCCTTGTTTAATCAGGAGACGCTACAGCA GAGGCTTCAGATGTTAATTGAGGGCGCCCAAGAGAGTTGGACATACGCCATATTCTGGCAGTGGTCGTACGACCCAAGCGGC GCCATCGTTTTCTGCTGGGCCGACGGCTACTTCAAAGGCGGCGGCGCCGCCGAGGAGGAGAAGCAAAGGAGCGCAGTTGA GCAGGAGGTGAGGAAAAAGGTGTTGCGGGAGCTGCACGCGCTCATTAACGGCGGCGGCACCGAAGCAGATCCCCCGGCTG AGGCGGAAGCGGAAGCGGATGAGGAGGTGACTGATGCGGAGTGGTTTgcggcgGTGTCGATGACGCACTCGTTTGGCGGCGGG CAGGGTGTGGCGGGGCAGGCGTATCTCAGCGGTTTGCCGGCTTGGCTGTCTGGGTCGCAGAAAATGGAGGGGTGTCATTGCG AGAGAGCTAAACAGGCGGCTCAGTTCGGCATTCAAACCATGGTATGTATTCCTACTCCCAATGGCGTTGTGGAATTGGGGTCT GTGGTTTTGCTTGCTCACAATTGGGAGCTGGTTCAACAGGCTAAGACCTCTTTTACTTTTTCGTCCTTTGGCTTGTGGGAAGAT CATGGAAATAATAACAACACTACATCTTCCGACAACAATACCAATCTCATCATCAACAACAACAACGACAACAACTTTTGTA ACAATAACCAGTCTCTGTGGGTTCCGGGTTCCCCTTTTTTCGGTGGCCATGCCCAGGAATCTCCCCTGGTGGATTTGGGTTTCT TCGGCGAAGAAACCAGGCACCCAAATCCCACCTTGACGCCAACCCCTTTGCCGGTTCAGAAATCCTTACCCGTCCTTGAAAA TGCCAACCCCTCTACTTTCCCTCCCAATAGGCAAATGCAAACGCCGCCGGAGGACGATCGGAGCCCTGTCCCATTTCCTGTCC AGAAGCCTGTAGTAGCCCACGAAGAGCTCCCCTCCTTTCCACTTGCGCAGAACACTGCCCTCTTAGAAGGATCCTTATCATTT GTGCATAAAACTGGGGTTTTCGATGATAAGTCTAGTGCCTTGCCGTATTCTGCAGCTCAGAAGCCTTCCCAAAATCACAATTCT TTGTTGTACCCTGCTGCTACTGCTCAGAAACCGTCCCAAAATCATAATCCTCTGTTGTACCCTACTGCTGCTGCTCAGAAGCTT CCCCAAAATCACAATCCTTTGTTGTATCCTGCTGCTGCGTCTCAAGAGCCTGCTCAAAATCATACTTCTTTGCTGTACCCTGAT GCTGCTAAGAAGCCTGCCCAAAATCACAATTCTTTGTTGTATTCTGCTATTCAGAAGCCTGCTGCTGTCTGTGACGACAATCAC AATTATATCCCATATTTGACTTCTCTGAAACCCATTGTCGATAGTGGGTCTTCGCAGTTTCCAAATCACACTACTGTGCAAGTTC TAGAACAGAACGGCAATGCATCACCATTCGCTGTTCAGAAGCCTATGGAGAACAAAGGGGTTTTTATACCAAAGGAGGAAG TGGACAATTCAAAACCCATGTTTCACTTTACAAATGGCAGTCTCCAGCGGGAAACTCTTGCAAACAGAGAATCTCAGAACCA AATGGAAGAGAAGATGAAAAAGCAAAAGACCGTTGATGAGAAGCATGGTTCCTTGCCTTCAATGTCTTCTGGTGCTTTCTTT GGTGGTGTTCGATCTGGTGCTGAGTCAGAGCATTCTGATGTTGAAGCTGCATCCTTCAAGGAAATGGAAGAAGCTGTTGTCGA GAAGAAGCCTCGGAAAAGAGGAAGGAAGCCTGCAAATGGCCGAGAAGAACCCTTGAATCATGTTGAAGCTGAACGCCAGC GGCGTGAGAAGCTGAATCAGAGGTTCTATGCACTTCGAGCAGTAGTGCCAAATGTGTCAAAGATGGATAAAGCTTCATTGCT TGGTGATGCTGTGTCGTATATTAATGAACTTAGAAGCAAAGTTCAAGAACTTGAGACTGAAAGGAAGGAGCTTGAATCCCAT GTTGAAGCTACCAAAAAGGAATTGCTATCGTCTCACTCTGGATTCTCTGGTGCCAACTTTGGGTTCGCTAAGGATCTGTCCGGT TCTTCTCAGGTGCCTGATAGCAAAGGATTTGGCACAAAGCAGTGCCCTGGATTAGATTTGGATGTCCGAGTTCTTGGTGCAGA AGCCATGGTCAGAATCCAGTCTGGTAAAAAGAATCATCCTGCGGCCAGATTGATGACAGCTTTTCAAGAGCTTGAACTTGAG GTTCATCATGCTAGTGTCTCAACTGTCAATGAGTTAATGCTTCAAAATGTTATTGTCAGGTTGCCAAAAAGTCTGTACACAGAA GAACAACTCAGCGCTGCCCTTTTTAAAAAGCTATCAAATCCAGGATTTTGTTAA $>$ EGL3

ATGGCTCCACCTGGAAAACAGAATGCACCGGCTGCTGTTGCAGATTTCACAGAAGCAGGTATAAGAATGAGGTTGAGACAG CAGATACAGGCAGCTGTACAAAGCATCCAGTGGACATACTCAGTTTTCTGGCAATTCTCTCCCCAACCAGGATTGCTTGAATG GGCTGATGGGTTTTACAATGGAGCCATAAAGACAAGGAAAACAGTACAACCCATGGAGCTGAGCAACGAAGAGCTGTGTGC ACAGAGAAGCCTGCAACTGAGGGAGCTGTTTGAATCTCTGTCTGCTGGAGAAAGCAATCAACCGGCCAGAAGACCCTGCGC TGCACTGTCTCCTGAAGACCTTACTGAATCCGAATGGTTCTATTTAATGTGCATGTCCTTCACCTTCGCTCCAGGAGTCGGATT ACCTGGGCGAGCACTTGCTCGACGTCATCATGTCTGGCTTTCTGACGCTCACGAAGCAGATAGTAAATTTTTTTCTCGGGCCAT TCTTGCCAAGAGTGCTCGAATCCAGACTGTTGTATGCATTCCCTTCATGGACGGCATCCTAGAGATTGGCTCAACAGACCAGA TACGTGAAGATGTGGGACTGGTGCAGCATGTAAAAAGCCTCCTTGTAGATCACTCAAAACCAGTGTGTTCCGAGCAATCCAC TTCAAATCCTCAAACTTCAGGAAATGCCACTCGTGTCCACTCTGAGGATCAGCTCAGCGTGCAGCCTCCTACAGGAATAGTTT TTGAAGAGCAGAACGTTAAGGCTAAAGAGTTTTCAGAAAGCCCATATGAAAACCCACACATTTCTGTGCAAGTATCTGGACT ACAAAGTGAGAAAACAGTTACAGAATTTGGACCGCACGGGAGCGAAATAATGGAGTTAGATATGTCGCCGGAAGATTGTTC CAATGATAATGATATTGGCTCTGAAGCACAGCTTCTATGTGGAAATAATTCTATGCATCAGAAGAGTTCAGTTCAGGCTTGGTC AGACATGAGCCATGGGCTTCAGTCACCAGGGCCTTACCAAGAGGAAGCAACCGAAGAAGAGAGCACCCATTATTCCAGAAC TGTTTCAATTATACTCCAACAGCAGCAGCTGCCACAAAGCGAATGGACTGACCCGACAACATTTCACAGTGACTATATTCCTG AGCAATGTGCATTCTCACCATGGACACCCAATGCTGGGTTTCTGCTGCAACACAAGTCCACTCCCAATCCACAGTGCATGCTC AAATACATATTGTTCACAGTGACTAGCCTCCATAGCAAAGACAAGGATAATGAAGGCTCCCCTAAACTAATCAGAGAAGGAG ACAACAATAGCAGCAGGTCTCGCAAAAATGGGCAGGATGACCTTACTGTAAACCATGTACTTGCAGAGCGTAGGAGGAGGG AGAAACTCAATGAGAAATTCATTATTCTGAGGACTCTGGTCCCATTTGTCACCAAGATGGACAAGGCCTCTATCTTGGGGGAT GCCATAGAGTATGTAAAGCAGCTGAGAAGGAAGATACAAGAGTTAGAAGTTCGCTCAAAACAAATGGAAGCCCAGATCAA AGGAAAAAATATCAGTCCTACCACAGATATAGCTCGCAAACAGTTAAACAATAAATCACAGGAAAAACTAGCCAGGCAAAA CAGTAGTGTAGGCAATCATAATCAGGTTACAGATCCGGAGCTAGTTAGCAGCTGCAGAAATTTCAGTGACACAGACCGATCC AAAGCATCCAGGTTTGAAAAGAGAAAAATCCGAGTTCTTGAAGGATCTGAGCCCCTCAAGATTGTAGAAGGGTGTGGCACA GATGTGCAGGTCTCTATAATTGAGAGAGAAGCACTGGTTGAGTTACAGTGCCCTTGGAGGGACAGTTTATTGCTTGATTTGAT GCAGACACTCAATTCTCTGTTTTTGGAAGCCTACTCTGTACAGTCTTCTATGGTTGATGCAACTTTTGTAGCAAAAATTAGAGC TAAGGTTAAAGAAGCACCCCTTGGGGCGAAACCCACAATTACTGAAGTCAAGACTGCCATTCAGTCATGCATTCCCATTCCA AGTTGA

$>$ TT8

ATGTGTGTGGCAATGGTTATGGCTTCTTCTTCTGGAACAGATGCCCAGGAAAGAAATAAACTCATTACAATACAGTTGGAGGC ACTTGTACAGAGCATACAGTGGACATATTCCATCTTCTGGCAGCTCTCTCCTCAAGAAGGGGTGCTGGAATGGAGCGACGGAT ACTACAATGGAGACATAAAGACGAGAAAGACTGTGCAGCCCATGGAGCTGAGTCCCGAACAGCTTTGCCTGCAGAGAAGC GTGCAATTGAGAGAGCTTTACGAGTCTCTATCTGCTGCGGAATCCAATCAGCCTGCGAGGAGGCCCTGCGCTTCCTTGTCTCC CGAAGATCTCACCGACACTGAGTGGTACTACTTGGTCTGCATGTCTTATACTTTCTCCCCTGCCGTGGGATTACCTGGACGGGC ATTTTCCCAGGGGCGTTTGGTGTGGCTATGCCAAGCTAATGAAGCTGATAGCAAAGTCTTCCCACGCGCTCTTCTTGCTAAGA GTGCTTGTATTCAGACTGTTGTATGCATTCCTCTCACAGATGGTGTATTGGAACTAGGCGCGACAGAACTGGTAAGAGAAGAT CCTGGGCTTGTCCAACACATGATGAGTTTTTGTATGGACCATCAGAGATTGATATGTTCGGATCAATCAACTTCCAGCCCTCAA GTCTCAGACAGAGATGATAAAGATCAAGTGGGATTGATCCCAGCCTTGATACCGCCTGACACTGTTGTATGTGTAGCTCAAAA TAATGGTGGATCCTCTATTACAGATTGTGGCCAATTTCTACAAAATTCCGAACATCTGCAACTTCCTTTGCAGTCATTCGATCCA AAAGACACTGAGTATCTCCAACTCAGAAACGAATCAATGCAAGTGGAAATTTATGAAGACTTCAAAACTACACCTCTCGAG GACTGTTCTAATATTCCTGGTCCTGAATTTACCTCAACAAGCAGGAAGGATTCCTTGCAGGTCCTACACAAGCACCAAAATGG CGACAATGTAAATGTAAATCCAAATTCACTTGAAAGTTGGTCATATATGCAGGAGGATGTGAGTCATGGACTTCAAGCATCTG GTATTTCAAGTGAATGTGTATCTCAGTCCATTGTTAACCCTCAGCTATGTACCTATTCAGCGCCGGAGATGCATGCTCTGTTGGG CTTGGATCAAAATTCAACTGATTTTAATAACATTCTGGAATCAGCACCTCAGACATTGGATGACACTCACTATTCCCGGACTTT GTCCAGTATATTAGACCAAAAAGCCAGTGAATTGTCAAATTTGGGTGCAATTAATTTGAAACCAAGGAAAAGTGGGAACTTA AAACTTTTGCAACAGGGCAGTTTTACAGAGTGGAAAAAGAAAGGCAACTGTGTCTTTGATAGAAAAACAGTAATTATCCCCC AGAAATTACTGAAAAAGGTCTTGCTTGGCATTCGTTATCTCCATAGCAAATACAGAGACCAAATTTCACTTAAACTCATGGAA GAAGACTTGGATAGCAAACTAGTGGGTCGGAGAATTGGGCATGATGATCTGAGTGTTAGCCATGTACTAGCAGAGCGCAGGC GGAGAGAAAAATTGAATGAGAAGTTTATAGCTCTCCGGTCTTTAGTTCCATTTGTTACTAAGATGGACAAGGCATCAATCTTA GCAGATGCCATAGAGTATCTACGGCAACTTCAGAGGCGGGTAGAGGAGTTGGAAACTTCCAACAAACAAATGGAGACTGAA ATTGGTATCAGAAATCCAAATTCTCTAAAGAGGATGTGTACAGAAGAGAGGATGACAAGACTTGATGGAAACATAAACAATG TGCAGTGTTTAGATGGGGAACTCAGCTGGATATTAACAGCTACAAAGCCAACTTGTAAAAGTCAAAGAGTGGAGAAGAGGA 
AATTTTGTGACTTGCAAGGATACCAAATTAATGTCTGTGCAGCATCAGATGTGCAGGTTTCATTCATTGAACAGGATAACATTT TAATAGAGATACAATGTCCTTGGAGAGATGATGTACTCCTTAATTTAATGCAGATATTGAGTGCTCTCCACTTTGACACATACTC TATCCAATCCTCTGTGGTGGATGATATGTTTGTTGCAGCACTCAAAGCTAAGGTCCGGGAATCCCTGGGTGGTAAAAGAGCAA GCATTGCTGAAGTTAAAGAAGCAGTTGAGTGCATTGTCTATAGAAGTTGA

\section{COI1 sequence}

\section{$>$ COI1-1}

ATGGCCGAAGACATAATTTCTGAGGAAGCTTTGGAGTGCGTGATGAACTACGTCGACGACCCCCGGGATCGAAGCGTGGTCT CTCAGGTCTGCAAGAAATGGTACTCAATTGACGCCCTTACAAGAAAGCACGTGACGGTGGCCTTCTGCTACACGATCCGGCC GTGCGATTTGACGCGAAGATTTAAGCGTCTGGAGTCCTTGAAGCTGAAGGGGAAGCCGAGAGCTGCTATGTTTAATCTTATTG AAGAAGACTGGGGGGGCTTTGCAAGGCCGTGGATAGAAGAGATCTCGGCTTCTTGTGTCTGCCTGAAGCGGCTTCATTTAAG GCGAATGATTGTTAACGATGATGATCTTGCGCAGCTCGTGAAGAGCCACGGTCATATGCTGCAGGCGGTGAAGCTGGAGAAG TGCTCTGGATTTTCGACACGCGGACTTCTTGCGATTGCTCGCGCTTGCAGATCTCTCAAGGTGCTCCTTTTAGAGGAAAGTTCC ATAAAAGATGAAGGTGGGGAATGGCTACACGAGCTTGCTCTCTCGAACTCTTCATTGGAAGTATTGAATTTCTATCTCACAGG CTTAGAAAGTACTGACTTGAGCGATCTTGAGTTACTGGCGACAAATTGTCGATCCTTAACCTTCTTAAGGGTAAATGAATGCG ATATTTTGGATCTAAGAGGTGTTCTGGAGAAAGCTACAGAATTGGAAGAATTCGGTGGTGGTTCGTTTTGTAACAGTGATGAG AATCCTTTAGAGGCAAATAAATATGAGAGAGTTAGATTTCCTTCAAAGTTGATATCACTTCTGGGGCTCAATTACATGAGTGAG ACTGAGCTGCCTTGTATACTTCCGCGGGCTTGCAACCTTAAGAAATTGGATTTACAGTTTACATTCTTGAGTACAGAAAATCAT TGCGAGTTAATCCGCATGTGTACTAATCTTGAAATTCTAGAGGTAAGAAATGTCATTGGAGACAGGGGGTTAGAAGTCGTTGC AAATCACTGCAAAAAGTTGAGGCGACTCAGGGTAGAGCCTGACGAAGAAGAAGGCTTAGATGATGAACAAGGTATAGTCTC TCACAGAGGGATTATTAACGTAGCTCAAGGCTGCCCCAATCTGGAATTCATTGCTGTCTATGTTTCAGATATAAGCAACGCAG CACTTGAGACTGTCGGTGAATTTTGCAAGAATCTGAAGGATTTTCGGTTGGTTCTGTTGGATAAAAAGGAACATATCACTGAT CTACCGCTTGATAATGGAGTCATGGCTTTGCTGCGTGGATGTCACAACTTAAACAGATTTGCATTATATGTGAGGCCTGGAGGA TTGACAGATACAGGGCTTGAATATATTGGAAAGTACAGTACAAATGTGAGGTGGATGCTTTTGGGATTTGCTGGAGAAAGTGA CCGGGGTCTCCTTGAGCTTGCAAATGGGTGCTCAAAACTGGAGAGGCTTGAAATGAGAGGTTGTTGTTTTAGCGAGACTGCA ATAGCATCTGCAGTGATTCATTTGAGATCACTGAAGTATATATGGGTTCAAGGTTATAAAGGAACAGCGACAGGAGAGAGAC TTATGGGCATGTCACGACCATTTTGGAACATAGAGTTTACCCCACCTCTCAGAATCACATTGGAGCGACATACGGATGGAGAT GTGATTGAAGAGCAACCAGCCCAGATACTAGCTTACTATTCTCTTGCTGGGATGAGAACAGATAACCCAGAGTCTGTGAACC TTTTGTCATCTCGGAGATAA

$>$ COI1-2

ATGGCCGAAGACATAATTTCTGAAGAAGCTTTGGAGTGCGTGATGAACTACGTCGACGACCCCCGGGATCGAAGCGTGGTCT CTCAGGTCTGCAAGAAATGGTACGCAATTGACGCCCTTACAAGAAAGCACGTGACGGTGGCCTTCTGCTACACGATCCGGCC GTGCGATTTGACGCGAAGATTTAAGCGTCTGGAGTCCTTGAAGCTGAAGGGGAAGCCGAGAGCTGCTATGTTTAATCTTATTG AAGAAGACTGGGGGGGCTATGCAAGGCCGTGGATAGAAGAGATCTCGGCTTCTTGTGTCTGCCTGAAGCGGCTTCATTTAAG GCGAATGATTGTTAACGATGATGATCTTGCGCAGCTCGTGAAGAGCCACGGTCATATGCTGCAGGCGGTGAAGCTGGAGAAG TGCTCTGGATTTTCGACACTTGGACTTCTTGCGATTGCTCGCGCTTGCAGATCTCTCAAGGTGCTCCTTTTAGAGGAAAGTTCA ATAAAAGATGAAGGTGGGGAATGGCTAAACGAGCTTGCTGTCTCGAACTCTTCATTGGAAGTATTGAATTTCTATCTCACAGG CTTAGAAAGTACTGACTTGAGCGATCTTGAGTTACTAGCGACAAATTGTCGATCCTTAACCGTCTTACGGGTAAATGAATGTG ATATTTTGGATCTAAGAGGTGTTCTGGAGAAAGCTACAGAATTGGAAGAATTCGGTGGTGGTTCGTTTTGTAACAGTGATGAG AATCCTTTAGAGGCAAATAAATATGAGAGAGTTAGATTTCCTTCAAAGTTGATATCACTTCTGGGGCTCAATTACATGAGTGAG ACTGAGCTGCCTTGTATACTTCCGCGGGCTTGCAACCTTAAGAAATTGGATTTACAGTTTACATTCTTGAGTACAGAAAATCAT TGCGAGTTAATCCGCATGTGTACTAATCTTGAAATTCTAGAGGTAAGAAATGTCATTGGAGACAGGGGGTTAGAAGTCGTTGC AAATCACTGCAAAAAGTTGAGGCGACTCAGGGTAGAGCCTGACGAAGAAGAAGGCTTAGATGATGAACAAGGTATAGTCTC TCACAGAGGGCTTATTAACGTAGCTCAAGGCTGCCCCAATCTGGAATTCATTGCTGTCTATGTTTCAGATATAAGCAACGCAG CACTTGAGACTGTCGGTGAATTTTGCAAGTATCTGAAGGATTTTCGGTTGGTTCTGTTGGATAAAAAGGAACAGATCACTGAT CTACCGCTTGATAATGGAGTCATGGCTTTGCTGCGTGGATGTCACAACTTAAACAGATTTGCATTATATGTGAGGCCTGGAGGA TTGACAGATACAGGGCTTGAATATATTGGAAAGTACAGTACAAATGTGAGGTGGATGCTTTTGGGATTTGCTGGAGAAAGTGA CCGGGGTCTCCTTGAGCTTGCAAATGGGTGCTCAAAACTGGAGAGGCTTGAAATGAGAGGTTGTTGTTTTAGCGAGACTGCA ATAGCATCTGCAGTGATTCATTTGATATCACTGAAGTATATATGGGTTCAAGGGTATAAAGGAACAGCGACAGGAGAGAGACT TATGGGCATGTCACGACCATTTTGGAACATAGAGTTTACCCCACCTCTCAGAATCACATTGGAGCGACATACGGATGGAGATG TGATTGAAGAGCAACCAGCCCAGATACTAGCTTACTATTCTCTTGCTGGGATGAGAACAGATAACCCAGAGTCTGTGAACCTT TTGTCATCTCGGAGATAA

\section{MED25 sequence}

\section{$>$ MED25}

ATGAATACGCAGGGAAGCTCAAGCGCTCACCAATTAATACTTGTGATCGAAGGAACTGCAGCCCTCGGCCCCGACTGGTCTC TGTTACGTTCTGAGTACATTGACAAAATTATCAGGTCTTTTTCTGGAAATGAGTTGGGTAGTCAGAGTTCTGGACCAATCATTG AAATGGCACTTATTGTTTTCTATAGTCATGGACCTCATAGTGTCTGTTTGGTGCAACGGAGTCCATGGACAAAAAAATTCGATC TTTTCCAAAAGTGGCTTTCAGATATCAATTTCTGTGGTGGCCTTGGGGAAGTTGCTATAGCAGAAGGCCTTGCTGAAGCATTA ACGATGTTTGCTCCTAGCCAGACTGTGACTCAGATGGCACAGGGGATGGACAGACAAAAGCACTGTGTGTTAGTTGCGGCAT CTAATCCACACCCTCTTGCAACTCCTGTTCCTCGACCATCCAGCCATACTTCAAGTCAGCAAAATGGAAATTCAGAATTTCAA ACACCGCAATGGCTTGCTGATGCAGAGACTGTTGCCAAGTCATATGCTCAGTCCTTTGTTTCTCTCTCAGTGATTGCGCCCAAA CAACTTCCCAAGCTTAGATCAGTTTACAATGCGGGCAAACGCAATCCATTGGCAACAGATGCAGTAATGGAAAATATAAAAC ATTCACAACACTTGGTTCTTCTTTCAGATGGTTTTATGGATGGTTGTTCTGCTCTAAGTTTCTCTGGAGCAACATCAGTAAATGT GAGTTCAAATTTGATAAAGACTGATTTAACATCTGCAGCTATGCACTCTGTTCCCCTTCAACCACAGAACACTGCTGTCAGAC CTTCATCTGGCCAAGCAGTGAATGGAGTTATGACCGGATATCCACAGGCACCAAGCAGAGCACTTCCTGTTGGGACTGTAAA GATGGAGGTTCCTTCAAGTGTATCTCTTACATCAGCATCTGGTTTGCCTCATATGACTTCTGGTTCACTTTTACATGGTACTTCA CAAGGAGGTCCTGGCCAATATTCTTCATCTTCAGGTCTTTCCGCTCAGGAGACAAAATCAAACATAGATGCAGCAGATGGAC AGGATTTTAAATCAGTTGTTGGTAACACATCACAACAACTTCGTACTGCAACAGCTTCCACCACAAATGTAAGCTTGTTGAAT AATCTATCACACTCGCGCTTGGGTTCAGCTGCTTTATCTGGAGCAAGTAATTTAGGGCTGCAAACAGGCAATGCTCCAGGGGG CATGCAAGCATCAAATATACTTGCTAGTGGAATGAGCTCAGCACCAATATCAACTCTTCAGAATGTTCAAGTGACAGGACAGT CAGGATTGACATCTGCTGGCACCTCTGTTGGCATGGTGGGGATTGGGCAGGTAGCTCCAAATGTTTCACAAAATTCATTTGGT GCCGGTGCCTCAAGTCTAGGTGCTGGACAACCAGTATCTTCTGGCAATACTGGTATTAATGGTGCACAGGGAGCAGTTGGTTT GGGACAATCCGTTCCAGGGATGGTAAATGGAGGAATGGCCACGGGAGCACAACTAGGACAGAGTGGAGTAGGAATAAGCC AAAATGTACTGGCTGGAATGGGGCCTGTTGGAGCAAGCTCTGGTGCAGGAACAATGGTACCCAAACCTAGTTTAAACCAGTC ACAACTAGGCAATTTACAAGGACTTGGAGTGGGTAACAGTTCTGCAGGTAATCTTCAGCTGCCATCAACTGCCACTACTTCTC AGCAGCCAGTGACAGCCAATGCAAAGTACACAAAAGTTTGGGAGGGTTTGCTTGGAGGTCAGAGGCAAGGGAAGCCAGTT CCTATTTGCAGGCTTGAGGCTTACAGGAATATAACTTCACCAGAAACACTTGCTGCAGACTGGCCAACATCAATGCAAATAAT CAGGCTTATTTCCCAGGAGCATGTAACCAACAAACACTACCAAGGAAAAGCAGAGTACCTGATCTTTAGACCAATGTCTCAA CATGGCTTCTTGGTGCAGCTTTCTGAAAAGAAGCTTTCTGCGGTGATACAACTGCCAAACCAGACACTGTTACTTTCCAGCTC CGACAGACCAGGCCGAATGATTGGCATGCTTTTCCCTGGGCAACCGCATATGCAACCGCAGCCTCATATGCAACAGCAGCCA CATCTGCAACAGCAACTTCAGCAGCAACAGCAGCTGCAGCAGCTCCAGCAGCAGCAGCAGCAGCTGCAGCAGCAACAACA GCTGCAGCAGCTGCAGCAGCAACAGCAACAGCCACAAGGAATGCCTTCGACAATGGGACAAGCATTTGCCCAAAGTCAAC TTCCCAATCAAGCCAGGCCACAATTTATATCACAAGGCCAATTCCAAGGCCACGGTCCTTCAAGTATGCCGGGATCTGGTTAC TTAAGTTAA 


\section{Appendix B. Promoter Sequence}

$>5$ alpha

CTAGATCACTGGGGAAGCGCCCCTTGCGTTGTCAGTCGTGTTCCGCCTGACTCTCCTCATCGCCGCTCCCGTTCCCGTGGGCA ACACAATTTTGATCGAGGTCCGGTTCATGGGCAGCGGAGCGCCTCCCCTCCTCGGCAGGGTCCTTCTCCGCCTCTTCTTGGGC TCCTCCCGCACCAGGGGTGTTTGGCCGGCTGGGTAGTGGCGGTTCTGGGTGGCAGGTGCCTGATCAATCCAAGTTCCCTCGGC TCTCCTTAGCCATCTCGCCCAACCAGCGGGGCACTGAGGAGGAGAAGCCGCAGACTGACGGCTGGACGCCGATTTCTGACA CCCCCGCCAACCCTCTTGGTGGGTGGGGTGACTGTCCGAAGAGCCCCCCGCAATAGCCACCTACCTCCATGTTCGCCACTGC CGCCTAGCTCCATGCCTGGCTTGGTTTCTGCAAATTTTGCTCCAACTTTTTTTGATTGATAAGTTTATCTTAATATGATTATGTAAT ATGTACCACTCGTAGCCTTTTTATGGCTACTTTTGCTCTGCATGTGGGGTGTGATCTTTTCCACCCGATTTTGTATGATTAGCATT GTACATTTCATCATTATTATATGGATATGCCTTTCAATCAAAAAAAAAAAAGTTCCTCGACATGTCTCTTTCAATGTATCCATTG AATTACCCACGTGCAATTGCCAGTTGAAAAATTGTTCAAATATAAAGCTACTTTAAATGATTTATCACATATAAGGAAGATAGC CTTATTAACTGATAATATGGAATCTAACGTTTGAAATGACCTTTGAAAACAGTCCAAGAAAACAATGACTTTTCACGTGGTAC CTAGAAACCGTATAAATCCATTATCCTGTGATCCTATATTTTTCGCGTGCTACGAACAAAGGCATATAAGTCCAACGACCCTAC ATTTTTGGCGTGGTGCAAACATCTCGGAGTAGAAAGTGTTATATTAACTCTTACAATCCATCCATCCATCCATCATATCACCAC GGATTTTCTCTGTTCTCTGTTCTCTTTCTTTTGGTTTTCTTCCAAAATGGATGCCCTGTATAAGAGTACTGTTGCAAAATTTAATG AAGTCACACAGCTGGACTGTTCCACCGAATTTTTTTCCATTGCTCTCTCATCTATTGCTGGT

$>7$ beta

ССТTTTTTTTTTTTAAAATAATTTTTTATTTTTTAAGTTTAAAGAAAAAATATTTTTGGGAAAGGTAAAAGTCGTATCCTTCATTGT AAGTTGCATTTATGTTTTAATATACATTATACTGAAATCCAATGCTAAATAAATAAATGATGAAATATTTAGTTTGAAGAGAGCA TAAGAGTGATCCATACCTTTTACGCTAATCCCATGATCCCTGCATTAGACGAGACCACATTAGCTTTAGGCATTCAGATAATAG TTACGGGATCATTCATGCTTACTCTTCCAACTTCGTTTTCCACATTTTGGACACAGATTGGTACGATGCTGGTAGAGGTAAATA ACTTATTTGGACACTATCTCATTGCATATAATTAGTTCTCATCCCAACATCCATGTCTTTATACACTGCCTCATTGAAGGGAATGT CGCAACTGTTATATATGATAGTGAACATTAAAATAAATCATACGTTTGGAGAAGACATATAAATTATAGAATCATGGGTGGAGG TTGGCATGGGTGGCATCAACCTGCATGGAGACCATTAGTTTGGCTTGAGAAAGAAGCATGTGTGGAAAAGGATGACTCTTTA ATTTCTGAGTGACTTGCACAAAGAATAAGAGACCAATGGCCAATGCAGCACTGCCTCTCTCACCATCAATCTTCCCCGAATAA TGTGCCTCTGAATCCTCCAACTTATACTTCTTCTTTTGCATTCATCAACAAATAGCAGCATATAGGCAGTTAATCATTCATCCCA AAAGTGTTGGACCCACTTCAGACATAGATCTAGACCCAGATCGGTATATTTTGAATATAGAAAAGTGAGGAGATAGTAGGATT GCAGTATATTTCTATGTGCGAGGTTGATTCAACCCATAATGGACTCTTTAGGACAGCTGAACAATCCTCATTCACAGAACTCCC TGCAATGTTCTATTTTTTCCATAGAAATAGGAGTGTATATCCCTCAAAGAATAATTGGAAACTACTCATGACGAATACAAGTCC AAAAAAATCGATTTTGATTTACCTGAGTGACTGAATTTGGATGGCCATGACAGCTCAATATACCGTTCCCTGTAATTATGTTCAT AAAATTGTATTTCAACGACCAGTTCCAAAAAAAAATCAATTTGATTTACCCAGAAGTGGGACTGATTTGATGGCATTGAAAG AGCTCCAATTATCACCGGTCCCTGTTAAGATATGTCATAAAATTGATTTCAACCGGAAATCATTGGCCCTACTTTCTAAAGTAA GAAGACTAAACTGATATTGTAATGAATTATGACATACTTTCTAAATTATCATGTGAAGCCCTACTTTCTAAATTATCATCTGAAG СССTTTTCTTAATATCCTCAAAAGGACAAGGCTCTACAGAATCTGAGTTGTCAAGAGGGATTTTGACATGTAGATGATGATGG AGAGTATCCTTCCACCGAGATATTGCCTTATCGATCTTTCATCTAGAACAACCACCATTTAAAGCAAGATGTCTCATTTCTGTTT AGAGTATCCTTCCACCGAGATATTGCCTTATCGATCTTTCATCTAGAACAACCACCATTTAAAGCAAGATGTCTCATTTCTGTTT
GGAAAAATTCCACAAAGAAATTTTAAATTTCCTATGGGTGAAGGCAATCTAAAAGATGAATAAAATTAGAACCTTTTAATGAA AAGATACAATCTATAGATCTCCTTCTACATAAGTGTATTTTGATTCATATAAAAAATCTTTTATAACTACAATCTTTGATGGAAA ACATGCTAACATTAAAATGACATCCCTTGGGCATACTTTGCAACTTATATTTATATCTCTATTGACATGTCCATAGCACAACGAC AATTACTAGCATTAATAATGTGTAGATGACAATAAGATTTGCAAAATCGACTACTATATTTGGAATCTAACGTTTGAAATGCTTT GAAACAGTATTGCAAAACACTTAAACAAGACGTTAATTTGTCGCGTGGGAAGTGATACCCACAATGTAAACAGGTTAGGTAA ACGACGATTTTTCACATGTTTCACTGCTCAGCATTATTCACATGCTCCCCTCAGTCCCCGTTTATCGTGCCGATTACACATATAA CACCACAAGGGAGATAGATCTATATAATCTTCTGCATCATCTCTTCTCCATTTCATCTCTGGTTTCTGCTCTGCCTTTTCTTACAA GAGTTTTCATAATGGATGCCCTTTCTCTTGTAAACAGCACAGTTGCAAAATTTAATGAGGTAACGCAGCTACAGGCTTCCCCT GCTATTCTGTCCAC

$>10$ beta

AAAGCTTAGAATATATTATTATAAACCTTATGAAATATACAATGTCTAACCCTCTATGGGCAATCAAAGATTGGTAGAGTTACA AGGGTGATGGGTTCGAAATTTTGGATACTTACTCTCAGGCCTCCAAAAGTCTCTCCGCAAAAATCTCCCTAAGTATCTAATTGG AAAGAGATTTATGGTTTTTTTCTACTCAATAGAAAATTTGAATTTCCTTCTAGAACACTCTCTCATGATTCTGCTCAAACTCAAA TTTGAATTGTTGTGCATTAATGGTGGCCAACTACCCTTGGTTTGGTAGTTGAGATAAGTCTATCTAAAACTCCATGTCATTGCTG AGTTGGCATCCTTTTGTGTACCTACCACCCTATCGTTTTTTGTCCTCAAGTAATTGCTCCACGCGCACTCTATTTTCCTTGTTGAA AGTTCCACGGACCCTTGTTTCATTCGATGCTTCAAAACCTTGGGTTAAGATATTCGCGCGCTAATGCGTAACTTGTCGTGTTCTT CCTTTTTTGTGCACTATCGTAAACTATTTTTCGCACCCTGACTACTTTGCTGACCTGCTCATTTTCCTCCGTACACCGCCTTAGCC AAAACTTCAAAATTCATGTAGTTCTGTAATTGTCCCGCAATGCTCCTACACCAAGAACACTCATGTGCAATTGCTAGTAATAAT AATAATTAAATAAATGAACGAGGAATTGTTTTTAAAATAACATTTAGTATCTCCGAATAATACATGTGTACGCACATCTCTGCA
AGTACCTAGCCTCCTAAATTTATGTAGCGTGTGAAATACTTGCACCTCGAATAATTTTGAGGTTCGTGTGGGAAATACATTTTG AATAATTTGAAAGTTTTTAATTATTAAAACATAATTCCAAACAGGAAATAGAACCGCTGGCGCACGTAGATTAAAAACGTGCA AAAGCACGGGAAAATTTTGAACGGGTCAAATACTTTAACTCATTGAAATTGAAAGCCCAGGGGTAGTTTTTCACATGCCAAC AAACCAAAAGGGCATATGGTATTTCAGCAGAGTCGCTAAGTAAAGTAGGCTAAGCACTCTGGAGCGCTTCATCTTGCAGCAT ATATAGTCTCTGCCATTCATCTTGCAGCATATGTACTACCATTCCTCTTTCCTATTCACTCCCTCCTCTCTCAAACCCACCTGCTC CAAATGGATAGCTTCATTTTTCTGAGAAGCATAGGAACAAAATTTGGGCAGCTGGAGTCTTCCCCTGCTATTCTTTCCCTTACC CTCGCACCTATTCTCGCCATTAT

$>13$ alpha

CCGTATAACTCCAAAAATATATCGCCCGGTAGGTGAGTCTTATTTCATTAAGGTGAAAGTTCGAACCTCTACGCGTGCCGATCA GTCAAAGCTCGGTCGAGGCTTTGGACTTCTGCTATGGACAGGAATCAGCTATGACATCGTCAGTATTGACTGCAGGCGGCCGC GATATCGAATTCGCGTGTCGGCCTTACTATAGGGCACGCGTGGTCGACGGCCCGGGCTGGTCTAAGAAACCAAACATTCCCAT CTATAATCAATCAACCATAATATTGCCATAAGGATCATGGTCTATCAGAGTTTATGTGTTGTTACAGACCAAGCCCACAAACTA TGTGAGATTAATTATTTGAATGAATGAGATTTTTTTTGATTGAAAGACAAATATCTTAATGCGTAAGACAAATTCATACACACA ATAGTTTGTGTTCTTAAGTTTTGTCAATGATTGATATATGTCTATTCTATTAGATTCACGAGGGCCTCATCCAAAGATGCACTAA AAGACATGGCCTAAGATAGTAAGACATATTTTATAGAGGTAATTACATGAATTTATTTATATCTAGGTATTATGAAAAGCTTATA GGAGGAGAGGGGAACGCCGAGCACACCTATCACCCTTAACATTTTAATAAAGATTTGTATTGTGCAATTTTATTGGATGGCCA AAACCTTAAAAGAAGCGCAAATTATATAGGTAAGCCCACGTTTGAAACATTGAAAGCAAAATATAAAAGATCACGCTTGAAA TAAAACAATCTCTTATTTCAAATGTGTTAATAAACCACAGTATTAGGTAGAGGGCGCGCATTATCACATGGAACTCGTTTATTC TCCCGCTTTCCCCAATTGCAATTATTTTATTCCTGTTATATATCACCGGTCCTCCTGTTATCCTCCACAAAAACTCTCGTCTCAGC TCTGCAAACTCAGTCTCTCTCCCGTTGCTCTGTTTTCTGCTCAGCAACTCCTTTCTGAAACGAAAATGGATGCCCTTAAGCAAT TGGAAGTTTCCCCTTCCATTCTTTTCGTTACCCTCGCAGTAATGGCAGGCATTATCCTCTTCTTCCGCTCTAAACG $>$ BAPT

CСTTATTTTGTATTCAAGGGTATAGTCCCTTTTTAAAAACTATTTGCCAATATTAAAAAAAACATTATATAAATACTAGTACATATTT ATTGAATTTTGTATATATGTACATTTACATATCTATTAAATTTTGATGTTTTGTAGATAATTGTTGATGTCTTGTTAAATTATTTTTTATI TTTACGAGAGTTATAGTTTAGTAGTTAGGATGATGACTTGTTAAGCTATTCACCAAAGTACACGTCCCTTTAGATATTTAATAGGA AGAGTCAAAATGGATAGAGTTGAGATCATTAGAATGATAGTTGGAGATAAACACAACCCTTAAGTTTGCATGAAATCACTACCT TCATGTTCTAAGTTAAATTTGTGTTTTCCTGTCCGTGACATGTCTAGTGAAACATGCATTCAAATTTCTCTTACATATACATTTTTTC TAACTACCCATTACAGTCAAGATCTACAAAGTCAAACAAGTATTGGCTACTCGCATTTTCAAATATCTCCCTCTAGGGTGACATCT TCACCTTCCCTAACACAATTTTTTTCCTTCATTCGATTGACATGCAAGTCATGCAATTTGTAGACGATATAGTTTTACATTGGATCC ATGTTTCCTCATTCATTTCCCTTTCACTTCTCCACCCTTGAATGCCATTTTCTACAATCCTCTTGATCTAATTTTACTTTTCCGTTATAT CCATTTTGGCATTCACATTGTACATTTAACATATGATTGCTAGTTCTTAGTCTTGTGAATACATCTGTGATCCATCTTGTGAATAAAT GGATCACAGAAGTAAGAAAAATATGTGTGACTGTGAATAAATATAAGCACTCGTGTCCAATCATCCATAATGAGCATTAAAAAA TGGAAATGGTAGATTTAATATATAATTCCTGGAAAACTGTTAATTAATGTCACTCAGGTTGAATATGGGGCCTCCATCGTTTCTTAA 
TTTTGGATGTTTCTCAGATGGAATCCAGACCCATGTTTCACTTCATCATTTTCCCTACATAAAAAATTATCCAGCTGCTCAATTCCC ATGTGAATATGATTGAGCGAGCCATGGTGACACCCTGCCTGCCTTCACCCAAAACAATCCTCCCTCTCTCCGCCATTGACAACAT GGAAAGAGCTTTTTCTAACGTATTGTTGATTTACATTGCCAACATGGACAGAGTCTCTGCAGATCCTG $>$ TAT

CCTCCCTTTTAGGGAGCAACTCAAACATATTGGAATTCCCAACATGTGCGTGTGCCCATCCTAAAACCTTCAAACATTTTTTTTTG GACTCGCCCTCATATTATAATTTTTATTTCATCGCTTTCACCATTATCTTGTCAATTAGAGGATGACTTTGTTTGGGGATTATTCTCA GTGGTCATCATACATTGCTTTTCTATGAAATTTCTTTACAATTATTAATTTATTTCAAGTATGAAAAGCTAGAAATGTAGTTATCATT AGTAGTAATTACATTTCTCAACAGTTTTATGTTAATGATAAAGTGGTTGTGATATATGTTGTTTATATCTAGATGTAGGCACAAGTT GGGAAAGTGGGATTGGAGTTCTAACACTTGTAGGGGCTATGAGATTATTGGGGAGCACTCCTTACTATGGTCTTGTGTCATTAGA TGTGCTACAACTGGGCCCTTCACCTTCTATTGCTAGGTGTAGCTTCCCCTATCCATAGTCATTTCTCAACCCTGCCACTGCAGTGG GGAGAGTGTACCCCACCACCTTGTGGAAAGTCCACTGCTCGTCCCTCTTAGTGGTGGTTCTTGGTGATGGTGTCATATGATTGGT TCTTCACCATTTAAGTGATGTATCATGATACCTTTAGCTATGCAAGACAATACTCAAGCTCAAGACAAATTGTCCGCCCTAACCCC CTTTAGGAGAGCCCTTTGCGTTGACTATCCTCGCACCCAAAGGATGAACCATTGCCTCTCCATAGTATTATTGTTGCTAGTATATA GCTCTCGCACTCCTTACTTTTTCATGTGTTCACCAAATTCTATTAATCGATAACATTTTTTAATGCTTTTAGTGGTGCGTTCCAATAA ATAATAATATTAAAGCCTACAAATAAGTAAGTGATATTAAGTGTAAATCTGTCATCCTTGTGAAATCCACAATCACGTGAATTAAA CTAACGTGGGAAATACACCATTTTATTTGAACAAAGACAAGCGTGTTAAAAAAAGAAACGTGTGATCCCAACGATGGGTTTTCG ACACACGTGAAAAATCAAATGAAAGGCAAATATCATTTTGACATTCTCTACATAAACCATCATTCTGCAACTCACTTTAAGCCGA CTCTTTCCCCTCCATTTCCATTTCCAGTTTCAGTTTCAGTACAGTTAGGATGGAGAAGACAGATTTACATGTCAATCTGATTGAGA AAATGATGGTTGGGCCATCCCTGCCTCCGCCCAAAACCACCCTGCAACTCTCCTCCATAGACAATCTGCCAGGGGTAAGAGGA AGCATTTTCAATGCCTTGTTAATTTACAACGCCTCTCCCTCTCCCACCATGATCTCTGCAGATCCTG $>$ TBT

TATTCTTTACTTTACTCACTCTTCTTTTCTTTTCAAACATGAACCGCGCTAACCTTACAATTTCAAAAAGAGTGAATTAAAATAA CCAAAGAGATAATTTTACCATTCCTTGTATGCTCTGAATCGCAAAGCTATACGGAATAAAATTATGCTCCTTAAATTATTTAACA TATTTAGTAGAGGAGAAAACTTGGATAAATTTAGCAGCAATTTTGGTCATAAGAAGTCAAAGTTATATGAGAGCTTATAAAGA AGGGATTTATATTGACAGGGTTTGAAGTTTTTATTTCAAAAAAATTCGAATTTTTGAATTTTTTGAAAGGAATTTTAATTCTGAG AAATCTTTAGCTTTTCGTAAAGGGGGAGAAAGGAAATTAAAATCATAAAGCTCACAGTATTTAAAAACTGAAAAATTTCTGGT TGTTTTAATGGTGGAGAAATGATTGCGTTCTTCTTCCACAGAAGACTTGGGTATTCCTTCAGGGCTTAACAAGATTAAAATGCG ATCGGGCCCCTCAAAAGAAGAGAATCAAATGAATTTAATTCCCTCCTATGTCCGTCTTACGAGTCATCACATTTCTGTGGAGA GTTAAACGACATTTCAGACATGTCATTTTCCTTGCGTAATAATATAAATGACATTTCCAACCTGTTAACCGTTCGCTGCCAAGT GTAAGGAAAAAAAAGGAAAAACAACTGTTAAAAATAGCTAGAAATGCGAAAACGTCCAAATGACTGTCAAAATAAAACAT ATTGGAAAAACGTCCGGGAGGCTGTCAAATTTAACATCGCTGTAGAAACGTCCGGAGATCCTTTACCATAGGGGGAACACCT TATCTCATACCAGTTCCGATAAATAAAATTGAAAGGAAAGCATGTGAAATTAATTGGTGATGTTGAATGGAAGGGAAGGCATG TGAAATTACTAACATGTGAAATTAATTGGTGATGTTGAATGGAAGGGAAAGCATGTGAAATTAATAACATGTGAAATTAATTG GTGATGTTAAATGCAAGGAAAGGCCGGCCCGGCATGTTCAGCAATAAAAAATAAAATTCTTAATAATTTATAGTTTTTGCCAG CACATGTGATAATCTCCGGCATATTTTTGACTTGTTAAAACCAACAACGTGAAGAGTTTAAAAAGCCCAAATAAACGTGTCAG CTGACTTAAAAAATCAATAATCATTTAAAACGTATGATATATCTCCACAAAAACTTCTTAAAAGTTAAAAAAATGTGTCACCA AGCAATCGAGTGAGTTGACTTTCTCAATTCTTATTAAAAGAGTACAAAATGTGTGAATAATCAAGCTGACAGCGACGTATGTT CTAATTTCTGGACAGTTACATTCCCTACATAAGCAGTTCAATTCACAAACCATTTCTGCAGTATTGAAGGAGAAGAGAGTCCA AATATCTACAATGGGCAGGTTCAATGTAGATATGATTGAGCGAGTGATCGTGGCGCCATGCCTTCCATCGCCCAAAAAAATCC TGCGCCTCTCCCCCATTGACAACAAAACCAGAGGACTAACCAACATATTATCAGTCT $>$ TS

AAATATTTCCCTTGTTCTGGTGTTTTAAAATGGGGATGTTACACTTGTGAGTTGATATTAGGTTTTTTAATAATCTCAAAATGAA CCGAGTATATTTCCCCATATTAAGGGGTATAGGGGGTTAAAGAATAAAGGTTTTTAAAAACATATTGGGGGTGGGTTACGTGG AGTGGTATGAAGGAGGGATGAGGAATCGTGGAGATGAGGAATCGTGGAGCAGATGAATAAGCCCTAAATTTACATCATTTTC ATTGTGTGTGTTTTTCAAAGCTGTGACATGGTTTCTAGAGAAGCTATATATTTCTTCATAGTAATGGCACTAGCTAACTCGCAAT AGCTAGGACATCTTTCATAGTATCAATTTTAAGGTATATTTTTTGTGATTTGATTCAATTTTTGTAGGAGAATGGATGTCATGAAG ATTAATAAATTTAACTGACAAGAATGCAGCCACACTTGACCTTTACCGTTACTATACAGAGTTTGATTAGTGTGACAAATATAG ATAGGAAAAAAGGAAGAGAGACATCAAATTACACATATTAAGGGTATCTACCCTTCAAGTCCAATATGATTTGCATCAAGTTT TAAAGTGGAGTTACATAATTAAATTTTGTGAAAATGTAAGATATTAAATCAAGACTGAAGAGGTGGACATAAAAAGGGTATAA TAAATGTCATAGGAAAATGAGTAGTTTGGATATGTGGTAAAATAAGGAGTATTTAATAATTGAAAATTTGTATTGGTCGTGGGT GTGACTTTTAAAAGATTGAGATATAGATTTGGAAATATGAGTATTATTTAATATCCTGTGAATGCATATGATAGGCAATAATTTG ATATAGCGAATCCCTCGTGACTTTCGAAGTTATCTTCAAAGTACAATGCCTCAGATATGTGCATCACCCCCCTTTCTCTTTCAAA AGTCATAAAATGACAAGTGTCCGTATGTGACTAACCTAGAAGAAAGAAGAACGTTGAGCTACGATTCAATCCCAACAATTTC TCTCTAC

GTGGACAGAATAGCAGGGGAAGCCTGTAGCTGGATTAGGCGAATGCCCTCCGTGACTTTCGAAAGTTATTCTCCAAAGTACA ATGCCTTCCAAAGAAATATGTGCATCACCCCCCTTCTCCTTTCAAAAGTTCATAAATGACAGTGTTCGTATTGTGACTACTAGA AGAAAGAAGACGTTGAGCTACGATCCATCCAACAATTTCTCTACCATTGAAAATTTCATGCTTCAACCCCCTTCCTTGTTACCC CTACTCTTCTTGCAATGTTTCAACTTCACACCTTTAACTTTAACTTAACTTTAACTTCTTCAATGCCTCCCTATCAACATATCCAT TACATTGCTTTGATCTAATTGCTAATATCTATAATACTCCTAAAAAATATAGTGGTGAACGACGAAGATCATGTTATGCATCAAC AACACGCCACTACCAATCTTCATCGTCCACTATCTACTTTGAAAATACCTCTCAACACAAAACTAAGCAATAACAAAGCACG AGTGCCACTAAAAGAGCGCCTAGACCATAAAAGTATAAAAAAATAACGCATTAAAGAATTAAAAACGATTTGTTTGCTAGTA ATTACCTAAAATAGAAACGAGTCAAGAGGGAATCTTTTTTTGGATTTTTCGAGACAATAATACTATTGTTTTTATATAACTATTT AAATATTTTTCTCTCTTCGAAAGTGCTTAAAAACGTGATGTTTTTAAGAGGGGGAAGATGGAACGTTGGGAAATGCTGCGGGC AAGTGTAATGGGCGTCGTCAGATCAAAACATGAATATCCGATTAAACACGTGATATGCGCCTGCCGCCTGACTTACTTCACTC ACTGCCACCTCTATTTAAAGTGGTTCTCCATACCATCGAAGACGTGTCCAACTCAGCATTTGATTTGGCATTTGCAATTTGGAA CTCGTAATTTCAGTAGTTCCCCTGCCTCTCTGCAGAAATGGCTCAGCTCTCATTTAATGCAGCGCTGAAGATGAATGCATTAGG GAACAAGGCAATCCACGATCCAACGAATTGCAGAGCCAAATCTGAGGGCCAAATGATGTGGGTTTGCTCCAAATCAGGGCG AACCAGAGTAAAAATGTCGAGAGGAAGTGGTGGTCCTGGT $>$ DBAT

CCTATAAAGAAGGGATAAACTACCTTGAGGGAAAGGCCCACACAAAAAGGGGGACCAAAACCAACCCCTTTTTTGTCCCTT ATTGCTTTATCCCCTTGTTATGTTCGTTTTCCATGTATTTTTGGATTTCTGCTTGATGATCTTGGGTCTCTTCCTTTTTTAGGTTTTC TCCCTTCCTCTATATATTTCATATCTTGTGATTGACCATTTTCATAGAAGTGTGTCTAGTTTTCTACCCCATAATCTTGTCACATCT CTATGAAGAATGTCCCGTACCTTTTCTAAGCACTTTTTACCCCCAAACTAATTTTATTGAGTTACATGCCTATTTTGGCTAGCTTT CCACCTATTGTTTGGCATCTGGAGTGCTAATAGGGTCCCAATTTAAATTCAAGAAGTCTATAATTACATAGGTGCATTTAGAGA CAAGAGGCAATCATACATTGAGTTATTTTCTTGGCACAAGAATGACATTGAATTTGTAAGGGCAAACTTCATTCTATTTATGAA GCATCCTTGTGCAAAGAATCCAATTCAAGCATTTAAAAAGATGGGGATTGTGGAACTTGCATGCACTCAATTAACTGTTAATT TGTACTGAGTGTTGCTTGCAATTTTTTCATATCATAAGGGGTTTGCTAATAATAGAAGAATAACATAGAACCATGTTAACATCCC ATATTGTTTATGAGTTATGAAAGGAGTTGAGTGCAAATTAGGGTATGGTAAAAGAGATCAAGTTCTCGGTGATCTACAACAGA AACAGAGAAATCGAGATACGCTTTCATATATTAGGGCATGAAGAAAACATTTACATCATAAGAATTACCTCAACCCAGACAA AACCATTTTTCATAGATTTGAGATCTCCACAAAACTAGACAAGTCAATTTTTTGGTTTGATTTTTTTGGGTGTTGTTTATATCACC CTCTAGACCAATTTCCACAAACTACCACTAACTAAAGCACATATAAAGACACTGATATCAACAGATTTACATCTCATCTTTCTA AACATTTAATTATTTTAATTATATTTCCACAATTTCCCACACTTTCTTTAATATTATATAATTAAGTATGAACCAATTGCATTTTTTT TCCAAACCCACTTCCCAATATCTTTGTCCTTACCAAAATTAGTTGTCAACAAGCGGAGAATCTAGATGATTAGTCACATTCTTA GGTGTGTGACTACAATATTAAACATTTCCTTTTAGGTATAAACAAATATTGCTTTGTTTTTTGTAATTTCCTAAGGCAAGCGTGC GAAATGCATGTTAAGTATATTTTATAAAATTTTGACCCACTTTGAAAACTAATTTAAAAAAAAACCAAATAATAATATAAATTG ACTCGAAAAACAGAAAACCTTTAGACATGTGAGAAATCCACCCATATTGAATTCGACGAAGACAGACCATATTTCATAGAAA ACGTGAACAATCAAGCGAAATACAGTCAGCAATTTCCTTTTCCCCTACATTAACAGTGATCCTGCACGTAATTTCCATCCTATT CTACGGTTGAAGATTTTCTGAGCTTGATCTGGGATGGCAGGCTCAACAGAATTTGTGGTAAGAAGCTTAGAGAGAGTGATGGT GGCTCCAAGCCAGCCATCGCCCAAAGCTTTCCTGCAGCTCTCCACCCTTGA 
$>$ GGPPS

ATCTTCCCTAATATATGATGGAGTTTGGAGCTTCTTTAGTGGATTCAATTTTTTAAATCCAATATGGTAGTGTAATTAGGTATCAA TAGAAAAATCACTACAAAAATAAAGACTTCCAGAATAAATATGTTAAAATTAATCTGTTACTATTTTCTAAAAATCTATACTAC TTTTACATATTATTTAATAAAGACATAAATACCCTACTCTTTCTAATTAAATTTAATTATACTTATCCCACATTTTTAAAATTCCAC ATTGAAATTAGTTAATCTACATTTTTAATCCCCTTGAAATTTGTTATTTCTACATTTTTATTCCCACATTGTAATTGGTTGATTCTAT GACCTTAAACTTATATTTATAATAAATTTTCCAACTATTTTTGAAGGTGGTGAATTACAAAATCTTAAACTTATATTATAATTTCA CATTAAAAATTTATGTAACTTACCTATTTAAAATATGATAGTATTATATAAATATATTTTGATAGTGCAAACAAAATAAATTCTCA CATTTGTAATCACAGTTTATCCCAAAATATTAGGTTTATTCCACATTTGAAATCCCCCATTGAAGTTTATTTATCCCACATTTGTA ATCCCGCATTGAAGAAAGTTCATTCCACGTTTTTAATGGTCAATGTTGTTGTCTGTTTATGGAGGATAAAATTTAACAGTCAAA TGTTTTGAAGATTCAAAGTAGATATACAACGGAAACCGGTCAATGGGTGTTATATTTTATCAACCGAAAAGCAGCCAAACCAT GGCATCTGGAATAAGTTATGGTTTGTCCTCACATTGTGGAAATCGGTTTCTCCTTACAATAGGGGACACGTCTCCATTGCAAAT GGTATCTACAAACTTTGTCGTTTTTGACATTCATCTTGCTACAAATATCTCTTATTCTGATTAACACATACACACCCAAAGGAAG AGCGGCAATCATTCTGTCATTTAAAGGTTTTCTGCACCGAAATCCTTTAATTTTAAGGTTTTCTGGAAGGTGTGTGTGAAAATTT GAAGAAAATTAACAAGACTGTAAGTGTTTTTATATATTTATGAGCTTTGATTTACTTGGGTGTTACTGAATTGGAACATACGTGC ATGTCGGAGCAAAGCAGCATATTTGAAATTTGTGGGTGTTCTTTGAGGTGTGTTGCGGATATAGATTTGATTGTTCAGAGTTAG TGTATTTTTTTTTTTGTTGGTTAGATTAATTGGGCAAGTTATAGAGAATGGCTTACACGGCAATGGCAGCAGGGACCCAGAGCT TGCAACTCCGCACTGTTGCTTCCTATC

>2alpha

ATCATCATGCAGGCCTTTGAACAAGTCAGAATTGAAAGGAATATGTTTGTTATTGTAATTTATGTACAATGATTCTTTGGTTTGA AAATAAACATCTAGATTTGTATGCATTTGCTCTGTTCTTCAATGTAGGTGAAAAATACTATCAAAGACACTAGAAACAGAGAG AAATGTAGAATAATTGAAAAAAAAGCACATTCAGAAATATAAACACTGCAAATAAATTAGAAGTTAAATCAATACAACACCG TTGTGCATAATTCTATTCCTCCTCTGAAACAAGTTGGAATTTTTTTCCTTCAATGTGTGTAATGATTAAAATTGCCTCAATAATAG AGGTCTTTATTATAAAAATAGATTTTCCTTACAATTTCAATGAGGATGTTAATGTTCAGGTCTCTAATTCCTGTCTCTGTCTCTAT TTTTGTTTCTGTTTTTCTACTCCTGTACTGCCTCACTCTATTGGCCGTGGAACCCATCATCAATTATTTTCGAAGGCAAACTTGAC CTCTGAATTCTTGCAAACAACTTAAAAAATTGTGGAAACACAATTCAAAAGGATAACAACTGTGTAAACGCAATTCAAAATG ATTTTTGTAGTTTAAGACTACACACTACTCAATGCAAAATTGGGACAATGAGCGAATTGAAAAGCTTTATTACAATAAAATAA CTCGAGCATCCAATAAATCACACCCCTTATCATCAACAACTACAAAAGTAATAACTACCCCTCTAAATATGCCTTTCAATGAG CCCACTGGGAATCAAGTCTTGCTGAGTGTAAGACCTGGAGACACCCGATACATAGAGTCAAACAATTGAGGCCATGATCGTG CCCTGGGTGATTTTGACGGCGTGGATGCCCCTTACCCGCAACTTCTTCACTGGTGCAGATATATAGATAGGGACGTCAATAGG CACCTATCTATACGATTTCAGTTCAAAATGATCCCAGATTAGATCTATATGGTCGCTCGATACCATCTCGGTTGGGTTGTTTAGTT AAAATCTCATGATATAAGATTTGCAAATGTATATGTACGAATTTAAAACAAATTTGAAATGTACAAATAGAACCTTTCAATGTA ATGCTTCATCTATATTCCTACACTTACATTATTAATCACACCCCTTATCAACATGTTAACGACTATACAAAAAACGTTTAAAAAA AAAAAACCAACCAAAAAACCGATGCAACCACCATCTCAGAGACGTTGCAACAATAACATGATTTCACCCAAATAATTAAAT ACCCCTCTAAATCAAATTACATATAACGTTCGGCGTTCACATTGCATTGTACACATGCGATTGCTAGTTTAGTGTACTTAACGTT GATTACAAAGCACGAAAATTATTCACGTGATCCTTTCGAATGCCACTGCCGTGCCACATGTAACGCGTTACCCATTATATTTAT TTGATAGAATTTATATGCATATAAATAGCTCATGCAAACTTTGCTTTTTCACATGGCGTTTTGACGATATATGAAGTAAGGTAGC TAAGTTCAACCTCTGCTATTTGCTGGTTTTCATTTTAATAATGGACGCCATGGATCTCACAGTTGCAAAGTTTAAGGAATTCAC GCAGCTACAGTCCTCTGCTATTCTTCTCACTGTTGTTTCTGGAATCATCGTCATCGTAATCC

$>14$ beta

AAAATCATCAAAACGTCTAAACTATCAAATTTTACTTCTGTTAGTCATTGATGAAATATAACATGGAACCTTGCCTCTCAATGG AATTTGTTTTCCAAAAAATGTTGTAATTAATTATTATTATATATAGTCAATTCGTATACTTTATATATTTATTATACTATTAATAGATT ATAGTTCATAATTATGTGTAGTTTTTTTTAACAAGCATATTTTTAATAATATAAATATAGATAAAATACAATTTTTATCATACAAAA

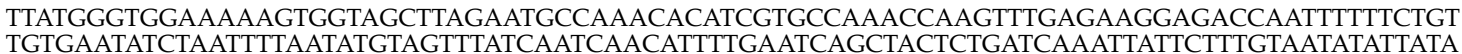
AGTAACGATCTGAAATATAGATTAGAAAGCGATAAAATTAATCCTAAAGTCTATTTATAAATCTTTAATATTTTTAATTTGTAATT TATTAGTTAATAATAGACATTTGAATATAAAATTAAGCTCACTCAAAACACTCCAAAAAGAAATATTAATAATACTGATATTTAT AATGAATCCGGTTTAATCAATAATTAATAGAATGTCTGGGCGGGACGAACGTCAAATTTCAGGTCCTCGTTCGAGCCATCGGA TTTTGGCAGTGGTCAAAGTTTGAGAAAGTGTTATATAAGCTCGACTATCCTTCCTTGTTTGATACGGATAATCTCTTCTGCTCTT TCCTTTCTCGTACTCCAATCCAAAATGGATGTCTTTTATCCGTTAAAAAGTACAGTAGCAAAATTAAACGGATGTTCCCCTGCA ATTATTTCCATTGTCCTCAGTGCTGTTGCTGGCATTG

$>$ PAM

ATCGGATGATGTCAATATATCTAAGTACATTACTATGGAACTTGCCAATCTGGAGGCAAAAAAGGTTTACATTTATTTAAAGCC AAGGATGGTAGATGAAAAATATGTGGAAGATGATTTGACAATCCCAGAAGCACCTAAAAGGAATAAAATAATGGGACAAAA GAGAACACATGAGGATACTCCTCCTAATGATGAAGATGGAAAGGAACAAGAAAAACTAATTCATGTTAAGAGCACAACTAC AAAGAAGGAAGCAACGTGCAAGGAGTTAGAACCAAGTGTGAGCATGTCTACAGGAAGTAAGAAGCCCATGAAGAATAGGT ATCTAGTGATGAAGAAACCAAAGATTGTATCTAGCGAGGAAAAAAAAGAGGTTGTGAGAAGTGAAGAGGAGGCTAAGACG AATGAAGAGGAGGTAATGTAGAAGGACTTGTTGGTTCATGATATTGAGGAGTCAGATATAGAAGAGCCCAATGAACATCATG AGGACATCCCAATCATGAAAATAGAGGTAGGAGGTAATGAGATGCCTGCTACTTAGATTTTGGTGGAGAGGAAGGAGCATAC TCAGGAGCATGAAGCTAAGCGGGAGATTGAGAGTGAACTTGAGCGCAATATTGAGCATGAAGTAGAGCACATTGTGGAACA TGACTTCAAGCATGGGGAAGAAGAAGGAAAGAGTGTTGATGGTGAAGAATAAGGAAAGAGTGTTAATGGTGAAGAAGAAA GTGTACATGAAAACCCCCTTATGATTGGTTTTCCAAAGGGGACCAGTGGAAAGCATAGGAAGCAAAGGCACATACCCAAGG GCTCTATGATGTAGTAAGTTGACAATCCTAATAATTTTTTTCTCTCAAGATATCTATGAGCATTTTTCTTTTTTAAAAAATAAAAA ATTGTAGTGCTTAGGTATATTCCTTTGGACTTTTTCCTTTCACATTTCATCACCTAGGCCTATCATTCCGCCTGTTATTGAAGAAA TTACTCGTGCTTTGAGAACATCGTACTCCCCCACATTCACAGTTGTTAAACATACTTAGTGTCGAATGTGGCTTTCACTGTTGCI TTAATCCGGCGAGATGGAATAATAGATCGTCATTCCACTTGAGTAAGAAGCCCGTGATCCCCTGTTAAAATCCGGCATGGTCG ACTGAGATGCTAACATCTACCTAAACAGACAAGACAACGAATACGTTCCCCGGGGGCCAGAACCATGTGTAAAAAAAATTA AATCATTGAGTGTTTCAGCATGTGATAAATGCAACGTCTCGCCCTTCCCTAGACGAATTCCGGGTTCACGTGTCTATATATATAA GTGCGACGCAACGATAAATTGCAGTGCAGCGTCCATTAGAGATTTTTCAGTTTTATCTCGCTCAAGTTTCAATCTTTTAATTTTA AACTTATTTTCCTTGCTCTGCAATGGGGTTTGCCGTGGAATCGCGTTCTCACGTAAAGGATATATTGGGGCTGATCAACACGTT CAACGAGGTGAAGAAAATTACAGTAGACGGTACGACCCCCATC

$>$ DBTNBT

CCTCCCTATTGGGCGCAAATTAGCGCATTACGGGATTGCTCCTCGGCTTTGCCCTTTTTGTGGGCATATTGAGACACTTAGGCAT ATGTTCTGGTTCTGCCCAAAAGCTCAGGGTATTTGGGTCTGGATCCACGATTTCTTTCGGCCATTTCGCCCAGCTCCATTTCTTT GGCAACATGTTTTGCTTGGGGATTCTATTTCCATTCCACATGCTTTTGCGCAAGTGTGGCATACTTTCAAAGTGGAGATCCTTTT TTTATATGGAAAGAACGCGATTCTGTTGTTTTTGCTAATTCCGCAGTTTCTTGCTTTCTTTCGCATTATGATAAGGCCATGCTTAT CTTGAATGTGTTCTTGCAGATCCAGGATCAGGCCCGCAAGGTTGAGCTGGAAGTGTGGCACCTCCAGACCCTCGTGGATCAT GGGCCAACGCTCCTTACACTGTGGCACAGGTGCCCCCCACCTCCCCTTCTAGGAGTGTTCGTTCCAGAGGTCATCGTGGCCA AGGCCAGTCGAATGAACGTACCCACCATCATCGCTCCCCCAGGTGGCAACCCGAGCATCGGCAGAGCTGTTATCAGGGTGGT TAAGGGTCCCCGCAGGGGCCCGTGCATCACAGTGGTTGTGGCTCCCGCCACCACTCCCCACCTCTGCGCAATGGGATGGATG GGATTCTGGCAAAGTCCCCCGGTTGGCTCTTGCCATATCTCTAATCTGCAAGACAACAAAGAAGAAGAAGAAGAAGAAGTT GGCACCGGCTGGCTGCCCCTTCCGGATGGAAATGATCTTCCCAGTGGTAACCTGGAGGATAAAGGGCCTCCACCACCGCCCC CTGCTGCTTTGTTTGTTGCGGCAGCTTAGTTTGTTTTCCTTATGTTTTGTGGCATTGTTGCCTTTTGATACTTTTGTCTCCTAGCCT TCGGCTAATTTGAACGTGAGGTGTGCCCTTTACCAACCATATTTTTTATGGATAGCTGTAATATTTTTATTAAATAATATAGTCTA TTTCATCAAAAAAAAATGATATCATTCTTTTTGCTTCTTGTTGTGCATGCTCTAAATGCACTAATTAATATACCTATAAATGTACA AACTTCTCGCATTCCAACAAAAACCCCAAACTCAATCTTAATAACTTTTTAACAAACCAAAACTATAAAATACAATGGAATGA GGATTTTATACTCGATTCCATTAATTTCTATTGGAAGCGTGTGAAATCCACTTAGATATTAAAAAAAACAAATAAACATGTGAC TTGAATCAAAAAGCCCACGTGAATAATCCAGCTGAATCCCCTACAAAAATGGATTACCCTACAGCTGAATCCCCATTCTCAGC CCATCGTTTCATCTTTGATCCAGTCATGGAGAAGGCAGGCTCATCAACAGAGTTCCATGTAAAGATCTCTGATCCAGTCATGG TGCCCCCCTGCATCCCTTCCCCCAAAACAATCCTCCAGCTCTCCGCCGTAGAC 


\section{Appendix C. Figures}

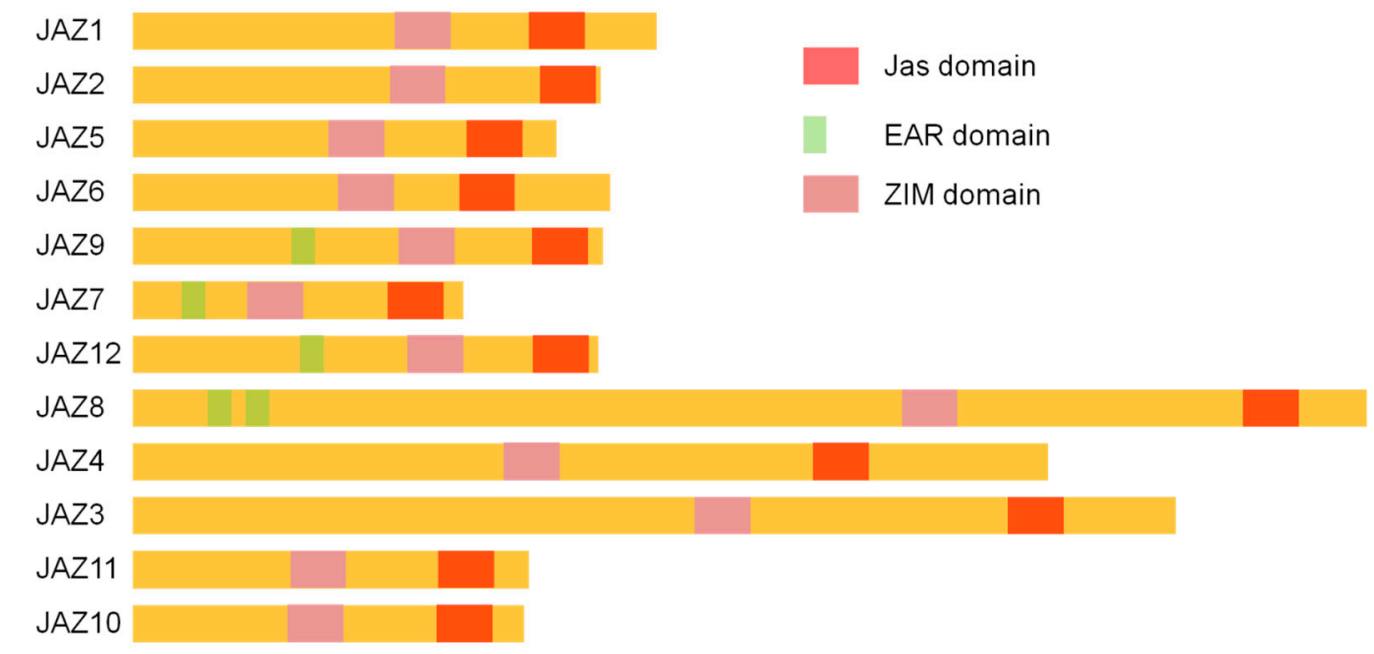

(a)

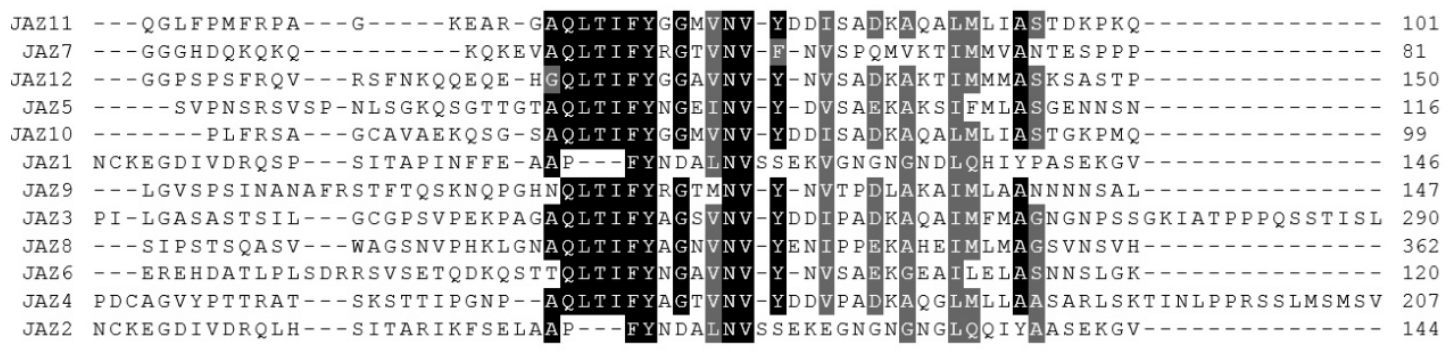

(b)

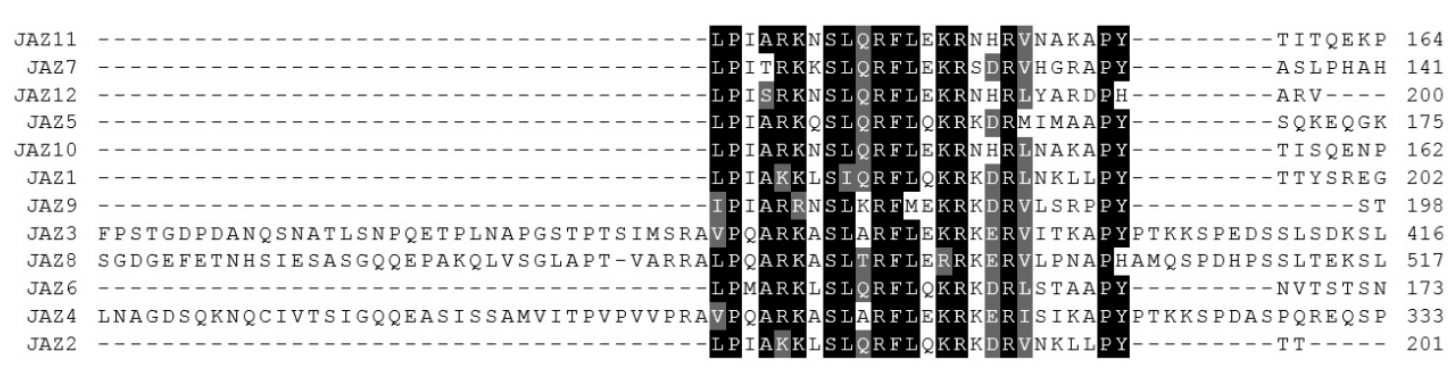

(c)

Figure A1. (a) schematic diagram of JAZ protein structure in T. media; (b) JAZ protein ZIM domain alignment map; (c) JAZ protein JAS domain alignment map in Taxus. 


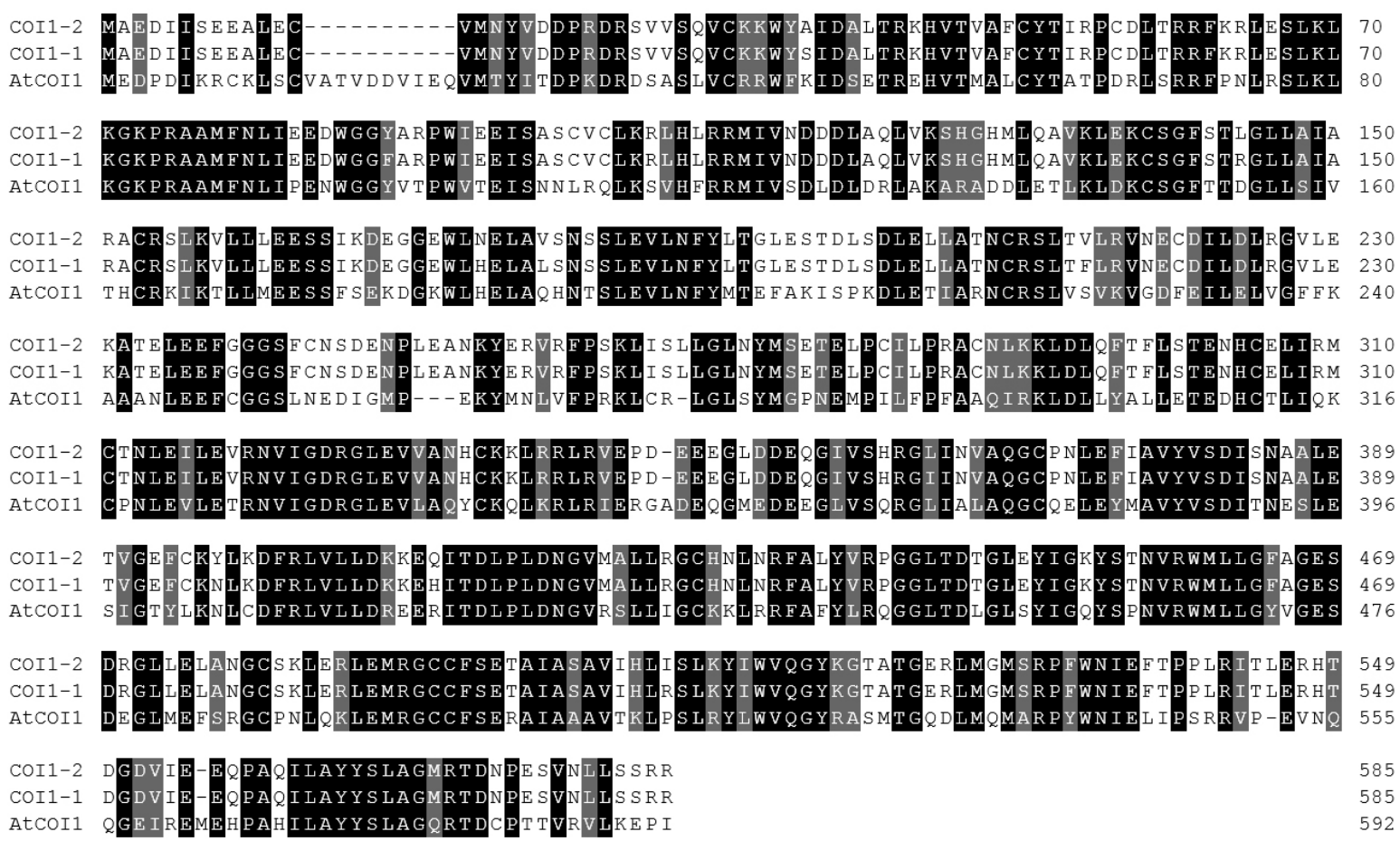

Figure A2. Results of the alignment analysis of Taxus COI1 and AtCOI1. 

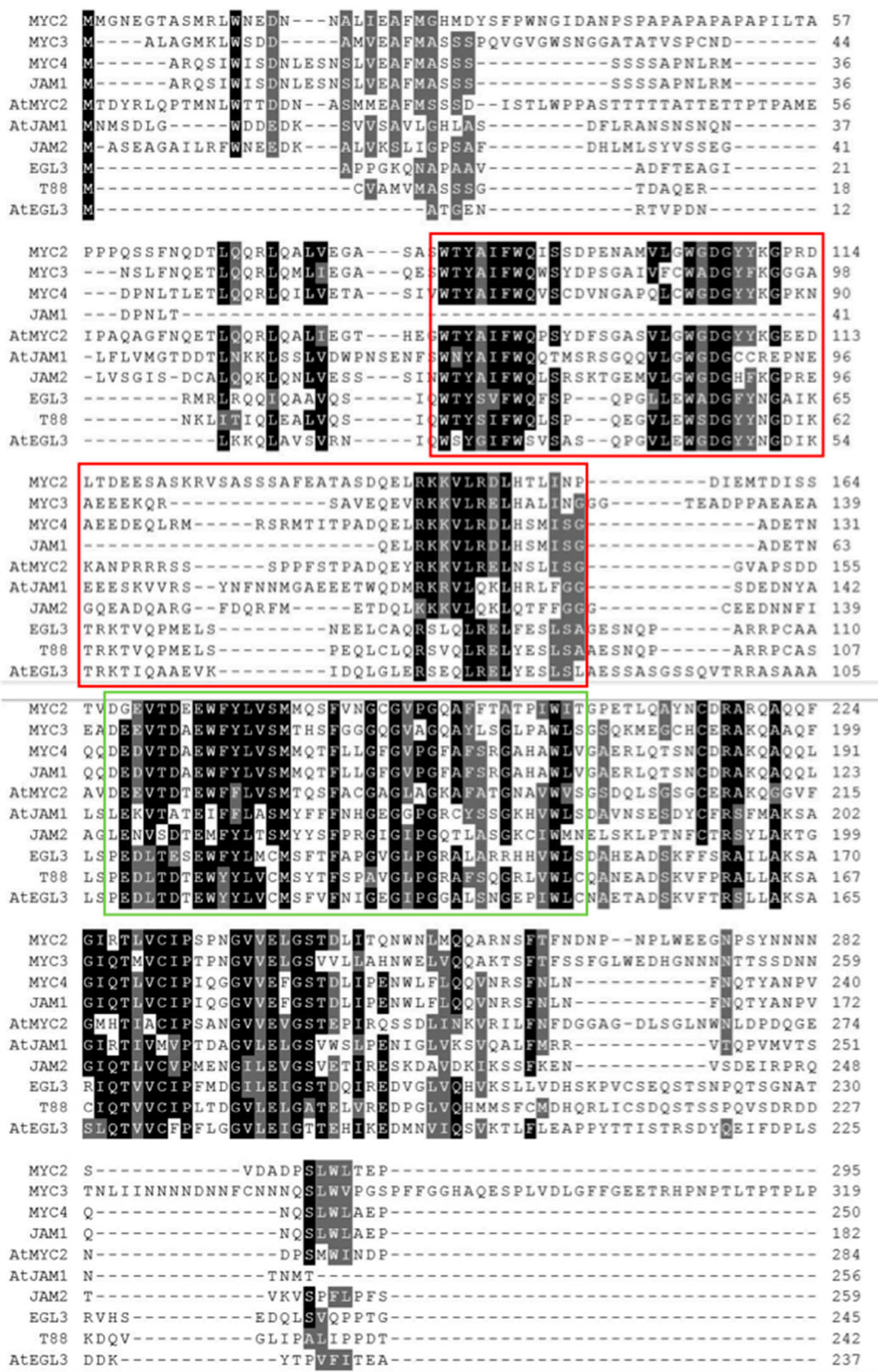

Figure A3. Cont. 


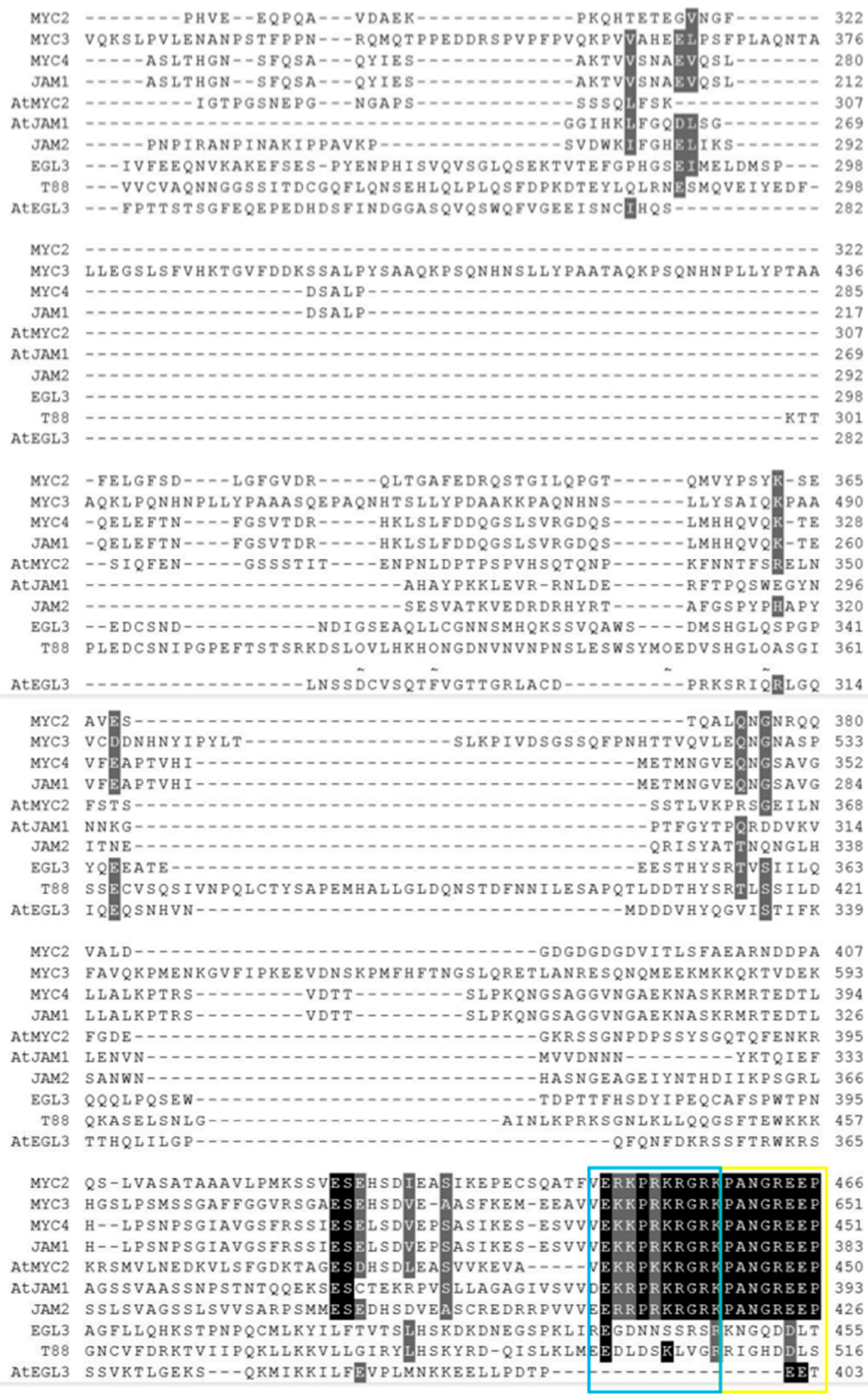

Figure A3. Cont. 

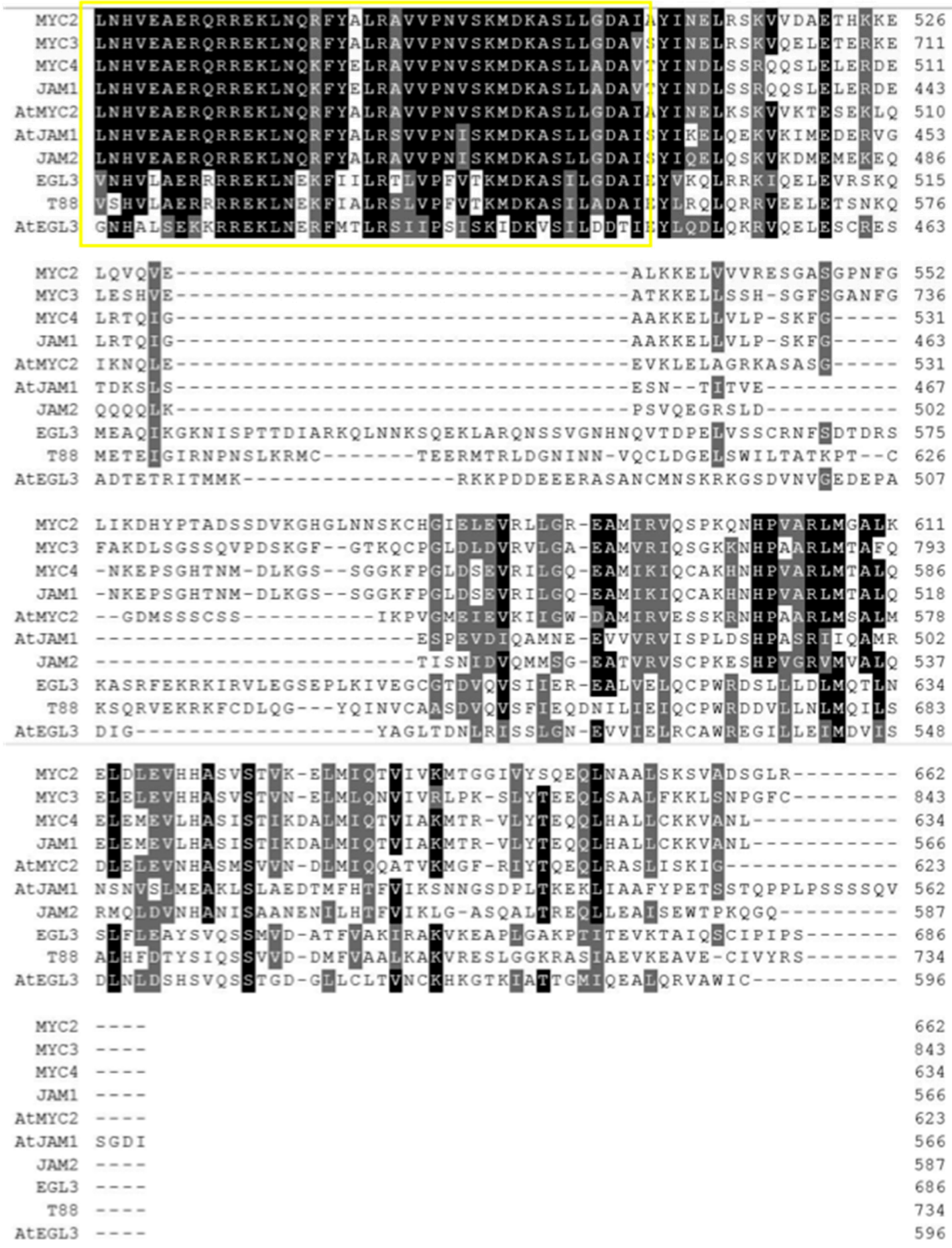

Figure A3. Sequence alignment and domain analysis of MYC transcription factors in Taxus. The red box indicates the JAZ binding region, the green box indicates the transcriptional activation region that binds to MED25, the blue box indicates the DNA binding region, and the yellow box indicates the region that binds to the bHLH class transcription factor. 


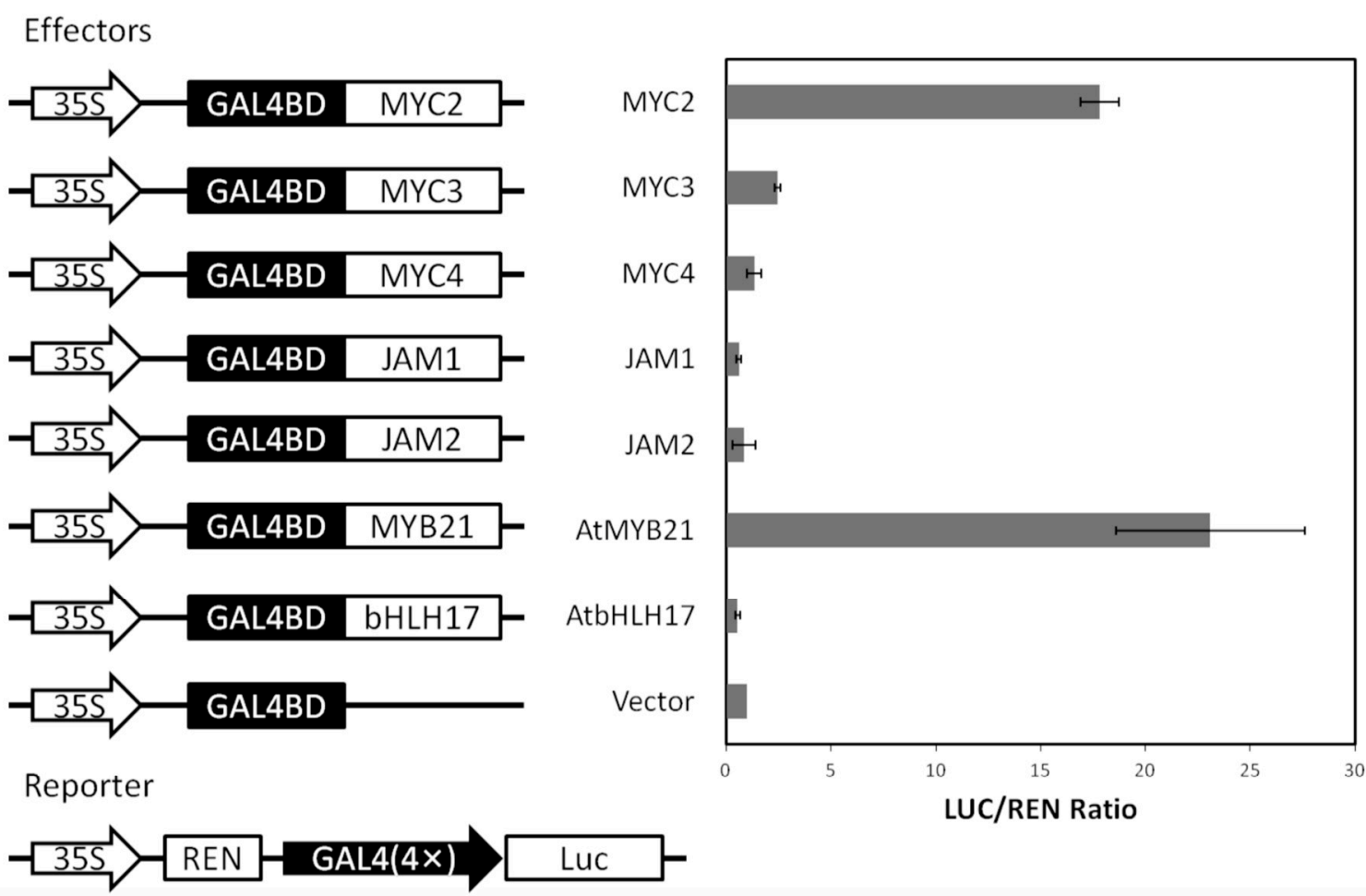

Figure A4. Detection of transcriptional activity of MYC-type transcription factors in protoplasts. The fusion protein overexpressing the MYC-type transcription factor and GAL4BD was used as the subtractor, the GAL4-regulated fluorescent protein LUC was used as the reporter gene, and the overexpressed red fluorescent protein REN was used as the internal reference to transfer into the Arabidopsis protoplasts to analyse the two kinds of fluorescence. The ratio between the signals. AtMYC21 and AtbHLH17 were used as positive and negative controls, respectively. Results are presented as a LUC/REN ratio. $n=3$ in all samples, error bars are $\mathrm{SE}\left({ }^{*} p=1.02 \times 10^{-11}\right)$. 


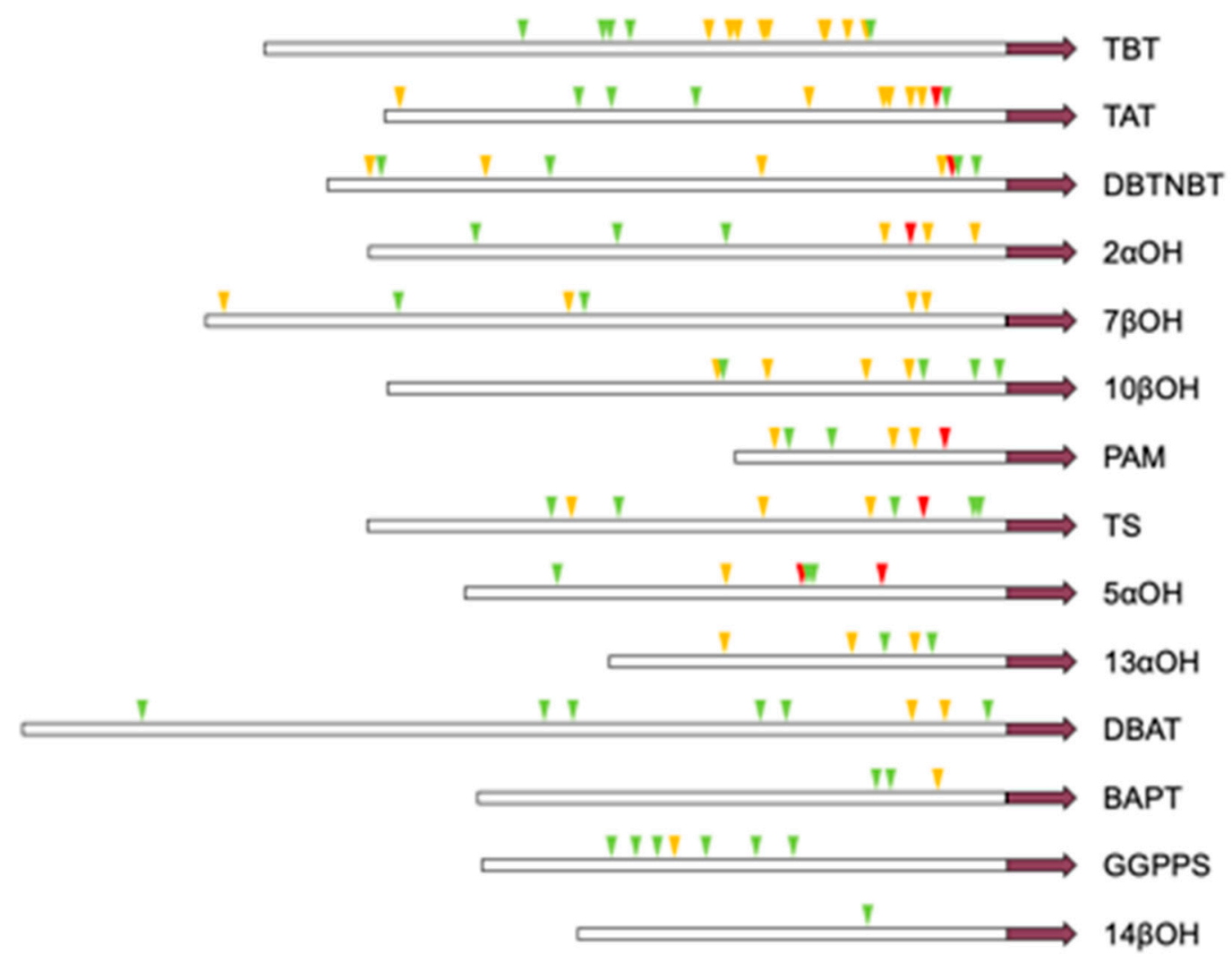

Figure A5. Location map of cis-elements in the paclitaxel biosynthetic pathway gene promoter sequence. The white rectangles show the promoter region of the corresponding gene in proportion, the dark red arrow indicates the start of the coding frame of the gene; the red triangle indicates the G-box, and the yellow triangle indicates the high affinity E-box or the variant G- Box. The green triangle indicates low affinity or no effect E-box.
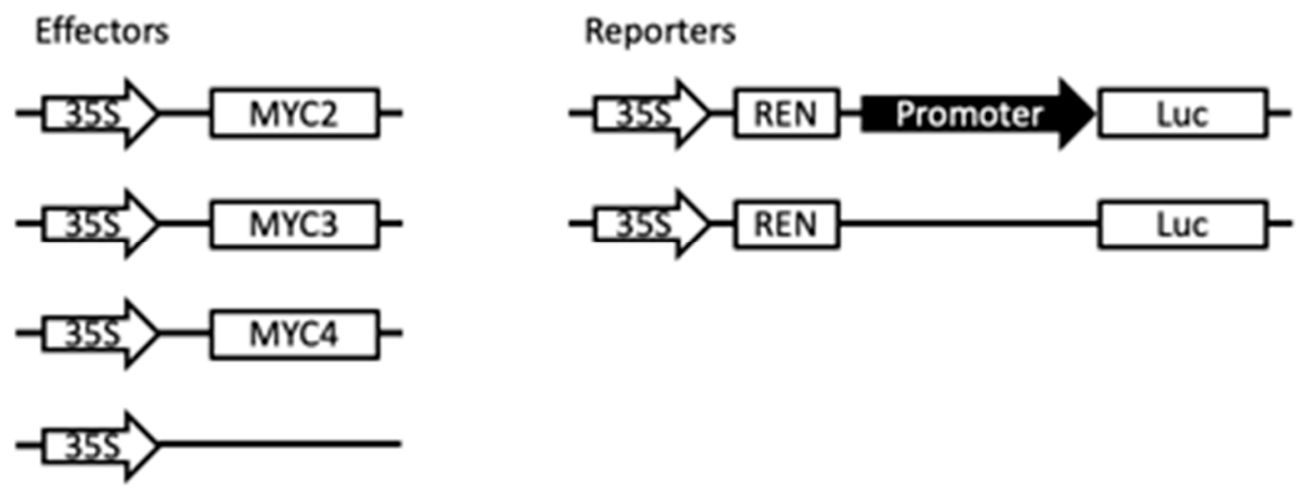

Figure A6. Detection of protoplasts MYC-like transcription factors regulate the activity of the paclitaxel biosynthetic pathway gene promoter. The plasmid overexpressing the MYC transcription factor was used as the effector, the fluorescent protein LUC regulated by the paclitaxel biosynthesis pathway gene promoter was used as the reporter gene, and the overexpressed red fluorescent protein REN was used as the internal reference to be transferred into the Arabidopsis protoplasts. The ratio between the fluorescent signals. Empty plasmid was used as a negative control.

\section{References}

1. Wani, M.C.; Horwitz, S.B. Nature as a remarkable chemist: A personal story of the discovery and development of Taxol. Anti-Cancer Drug 2014, 25, 482-487. [CrossRef] [PubMed] 
2. Lenka, S.K.; Nims, N.E.; Vongpaseuth, K.; Boshar, R.A.; Roberts, S.C.; Walker, E.L. Jasmonate-responsive expression of paclitaxel biosynthesis genes in Taxus cuspidata cultured cells is negatively regulated by the bHLH transcription factors TCJAMYC1, TcJAMYC2, and TcJAMYC4. Front. Plant Sci. 2015, 6, 115.

3. Li, S.; Zhang, P.; Zhang, M.; Fu, C.; Yu, L. Functional analysis of a WRKY transcription factor involved in transcriptional activation of the DBAT gene in Taxus chinensis. Plant Biol. 2013, 15, 19-26. [CrossRef]

4. Zhang, M.; Li, S.T.; Nie, L.; Chen, Q.P.; Xu, X.P.; Yu, L.J.; Fu, C. Two jasmonate-responsive factors, TcERF12 and TcERF15, respectively act as repressor and activator of tasy gene of taxol biosynthesis in Taxus chinensis. Plant Mol. Biol. 2015, 89, 463-473. [CrossRef]

5. Croteau, R.; Ketchum, R.E.; Long, R.M.; Kaspera, R.; Wildung, M.R. Taxol biosynthesis and molecular genetics. Phytochem. Rev. 2006, 5, 75-97. [CrossRef] [PubMed]

6. Ajikumar, P.K.; Xiao, W.H.; Tyo, K.E.J.; Wang, Y.; Simeon, F.; Leonard, E.; Mucha, O.; Phon, T.H.; Pfeifer, B.; Stephanopoulos, G. Isoprenoid Pathway Optimization for Taxol Precursor Overproduction in Escherichia coli. Science 2010, 330, 70-74. [CrossRef] [PubMed]

7. Walker, K.; Croteau, R. Taxol biosynthetic genes. Phytochemistry 2001, 58, 1-7. [CrossRef]

8. Hampel, D.; Mau, C.J.D.; Croteau, R.B. Taxol biosynthesis: Identification and characterization of two acetyl CoA: Taxoid-O-acetyl transferases that divert pathway flux away from Taxol production. Arch. Biochem. Biophys. 2009, 487, 91-97. [CrossRef] [PubMed]

9. Patra, B.; Schluttenhofer, C.; Wu, Y.M.; Pattanaik, S.; Yuan, L. Transcriptional regulation of secondary metabolite biosynthesis in plants. BBA-Gene Regul. Mech. 2013, 1829, 1236-1247. [CrossRef]

10. Li, S.T.; Zhang, P.; Zhang, M.; Fu, C.H.; Zhao, C.F.; Dong, Y.S.; Guo, A.Y.; Yu, L.J. Transcriptional profile of Taxus chinensis cells in response to methyl jasmonate. BMC Genom. 2012, 13, 295. [CrossRef]

11. Sun, G.L.; Yang, Y.F.; Xie, F.L.; Wen, J.F.; Wu, J.Q.; Wilson, I.W.; Tang, Q.; Liu, H.; Qiu, D. Deep Sequencing Reveals Transcriptome Re-Programming of Taxus $\times$ media Cells to the Elicitation with Methyl Jasmonate. PLOS ONE 2013, 8, e62865.

12. Dai, S.H.; Zheng, P.; Marmey, P.; Zhang, S.P.; Tian, W.Z.; Chen, S.Y.; Beachy, R.N.; Fauquet, C. Comparative analysis of transgenic rice plants obtained by Agrobacterium-mediated transformation and particle bombardment. Mol. Breed. 2001, 7, 25-33. [CrossRef]

13. Mao, R.; Chen, J.; Chen, Y.; Guo, Z. Identification of early jasmonate-responsive genes in Taxus 3 media cells by analyzing time series digital gene expression data. Physiol. Mol. Biol. Plants 2018, 24, 715-727. [CrossRef]

14. Chen, R.; Jiang, H.L.; Li, L.; Zhai, Q.Z.; Qi, L.L.; Zhou, W.K.; Liu, X.; Li, H.; Zheng, W.; Sun, J.; et al. The Arabidopsis Mediator Subunit MED25 Differentially Regulates Jasmonate and Abscisic Acid Signaling through Interacting with the MYC2 and ABI5 Transcription Factors. Plant Cell 2012, 24, 2898-2916. [CrossRef]

15. Fernandez-Calvo, P.; Chini, A.; Fernandez-Barbero, G.; Chico, J.M.; Gimenez-Ibanez, S.; Geerinck, J.; Eeckhout, D.; Schweizer, F.; Godoy, M.; Franco-Zorrilla, J.M.; et al. The Arabidopsis bHLH Transcription Factors MYC3 and MYC4 Are Targets of JAZ Repressors and Act Additively with MYC2 in the Activation of Jasmonate Responses. Plant Cell 2011, 23, 701-715. [CrossRef]

16. Sasaki-Sekimoto, Y.; Jikumaru, Y.; Obayashi, T.; Saito, H.; Masuda, S.; Kamiya, Y.; Ohta, H.; Shirasu, K. Basic Helix-Loop-Helix Transcription Factors JASMONATE-ASSOCIATED MYC2-LIKE1 (JAM1), JAM2, and JAM3 Are Negative Regulators of Jasmonate Responses in Arabidopsis. Plant Physiol. 2013, 163, 291-304. [CrossRef]

17. Wani, M.C.; Taylor, H.L.; Wall, M.E.; Coggon, P.; Mcphail, A.T. Plant Antitumor Agents. 6. Isolation and Structure of Taxol, a Novel Antileukemic and Antitumor Agent from Taxus-Brevifolia. J. Am. Chem. Soc. 1971, 93, 2325-2327. [CrossRef]

18. Hong, H.; Xiao, H.; Yuan, H.; Zhai, J.; Huang, X. Cloning and characterisation of JAZ gene family in Hevea brasiliensis. Plant Biol. 2015, 17, 618-624. [CrossRef]

19. Stitz, M.; Hartl, M.; Baldwin, I.T.; Gaquerel, E. Jasmonoyl-L-Isoleucine Coordinates Metabolic Networks Required for Anthesis and Floral Attractant Emission in Wild Tobacco (Nicotiana attenuata). Plant Cell 2014, 26, 3964-3983. [CrossRef]

20. Chini, A.; Fonseca, S.; Fernandez, G.; Adie, B.; Chico, J.M.; Lorenzo, O.; García-Casado, G.; López-Vidriero, I.; Lozano, F.M.; Ponce, M.R.; et al. The JAZ family of repressors is the missing link in jasmonate signalling. Nature 2007, 448, 666-671. [CrossRef] 
21. Thines, B.; Katsir, L.; Melotto, M.; Niu, Y.; Mandaokar, A.; Liu, G.; Garcia-Casado, G.; López-Vidriero, I.; Lozano, F.M.; Ponce, M.R.; et al. JAZ repressor proteins are targets of the SCF(COI1) complex during jasmonate signalling. Nature 2007, 448, 661-665. [CrossRef]

22. Wager, A.; Browse, J. Social network: JAZ protein interactions expand our knowledge of jasmonate signaling. Front. Plant Sci. 2012, 3, 41. [CrossRef]

23. Thireault, C.; Shyu, C.; Yoshida, Y.; St Aubin, B.; Campos, M.L.; Howe, G.A. Repression of jasmonate signaling by a non-TIFY JAZ protein in Arabidopsis. Plant J. 2015, 82, 669-679. [CrossRef]

24. Pauwels, L.; Goossens, A. The JAZ Proteins: A Crucial Interface in the Jasmonate Signaling Cascade. Plant Cell 2011, 23, 3089-3100. [CrossRef]

25. Chen, J.; Yan, Y.X.; Guo, Z.G. Identification of hydrogen peroxide responsive ESTs involved in phenylethanoid glycoside biosyn-thesis in Cistanche salsa cell culture. Biol. Plant. 2015, 59, 695-700. [CrossRef]

26. Yoo, S.D.; Cho, Y.H.; Sheen, J. Arabidopsis mesophyll protoplasts: A versatile cell system for transient gene expression analysis. Nat. Protoc. 2007, 2, 1565-1572. [CrossRef]

(C) 2019 by the authors. Licensee MDPI, Basel, Switzerland. This article is an open access article distributed under the terms and conditions of the Creative Commons Attribution (CC BY) license (http://creativecommons.org/licenses/by/4.0/). 